\title{
Cell-free gene expression dynamics in synthetic cell populations
}

David T. Gonzales ${ }^{\mathrm{a}, \mathrm{b}}$, Naresh Yandrapalli ${ }^{\mathrm{c}}$, Tom Robinson ${ }^{\mathrm{c}}$, Christoph Zechner $^{\mathrm{a}, \mathrm{b}, \mathrm{d},{ }^{*}, \mathrm{~T}-Y}$ Dora Tang ${ }^{\mathrm{a}, \mathrm{b}, \mathrm{d},{ }^{*}}$

${ }^{a}$ Max Planck Institute of Molecular Cell Biology and Genetics, 01307 Dresden, Germany

${ }^{\mathrm{b}}$ Center for Systems Biology Dresden, 01307 Dresden, Germany

${ }^{c}$ Max Planck Institute of Colloids and Interfaces, 14476 Potsdam, Germany

${ }^{\mathrm{d}}$ Physics of Life, Cluster of Excellence, TU Dresden, 01603 Dresden, Germany

* Correspondence to: zechner@mpi-cbg.de and tang@mpi-cbg.de

\section{Contents}

1. Chemicals, materials, and equipment

2. Plasmid construction

3. mRNA expression and purification

4. Protein expression and purification

5. Protein and mRNA calibration

6. Bulk cell-free expression experiments

7. mCherry maturation

8. Resource-limited gene expression models

9. Parameter estimation, model selection, and profile likelihoods

10. Microfluidic chip fabrication and pretreatment

11. Encapsulation of CFES in liposomes using microfluidics

12. Inverse emulsion phase transfer method

13. Image acquisition and analysis

14. DNA titration series in synthetic cell populations

15. Two plasmids in a synthetic cell population

16. Dilution of CFES outer solution in synthetic cell populations 


\section{Chemicals, materials, and equipment}

Table S1. Chemical list.

\begin{tabular}{|c|c|c|c|}
\hline Name & MW (g/mole) & Supplier & Catalog No. \\
\hline \multicolumn{4}{|l|}{ Amino acids } \\
\hline L-Alanine (A) & 89.09 & Sigma, USA & A7627 \\
\hline L-Arginine $(\mathrm{R})$ & 174.20 & Sigma, USA & A5006 \\
\hline L-Asparagine $(\mathrm{N})$ & 132.12 & Sigma, USA & A0884 \\
\hline L-Aspartic acid (D) & 133.10 & Sigma, USA & A9256 \\
\hline L-Cysteine (C) & 121.16 & Sigma, USA & W326305 \\
\hline L-Glutamic acid (E) & 147.13 & Sigma, USA & G1251 \\
\hline L-Glutamine (Q) & 146.14 & Sigma, USA & G3126 \\
\hline Glycine (G) & 75.07 & Sigma, USA & G7126 \\
\hline L-Histidine (H) & 155.15 & Sigma, USA & H8000 \\
\hline L-Isoleucine (I) & 131.17 & Sigma, USA & $\mathrm{I} 2752$ \\
\hline L-Leucine (L) & 131.17 & Sigma, USA & L8000 \\
\hline L-Lysine (K) & 146.19 & Sigma, USA & L5501 \\
\hline L-Methionine (M) & 149.21 & Sigma, USA & M9625 \\
\hline L-Phenylalanine $(\mathrm{F})$ & 165.19 & Sigma, USA & P2126 \\
\hline L-Proline (P) & 115.13 & Sigma, USA & P0380 \\
\hline L-Serine (S) & 105.09 & Sigma, USA & $\mathrm{S} 4500$ \\
\hline L-Threonine $(\mathrm{T})$ & 119.12 & Sigma, USA & T8625 \\
\hline L-Tryptophan (W) & 204.23 & Sigma, USA & T0254 \\
\hline L-Tyrosine (Y) & 181.19 & Sigma, USA & T3754 \\
\hline L-Valine (V) & 117.15 & Sigma, USA & V0500 \\
\hline \multicolumn{4}{|l|}{ NTPS } \\
\hline $\begin{array}{l}\text { ATP (Adenosine 5'-triphosphate disodium salt } \\
\text { hydrate) }\end{array}$ & 551.14 & Sigma, USA & A26209 \\
\hline $\begin{array}{l}\text { CTP (Cytidine } 5 \text { '-triphosphate disodium salt } \\
\text { hydrate) }\end{array}$ & 527.12 & Sigma, USA & 30320 \\
\hline $\begin{array}{l}\text { GTP (Guanosine } 5 \text { '-triphosphate sodium salt } \\
\text { hydrate) }\end{array}$ & 523.18 & Roche, Switzerland & 10106399001 \\
\hline $\begin{array}{l}\text { UTP (Uridine 5'-triphosphate trisodium salt } \\
\text { dihydrate) }\end{array}$ & 586.12 & Sigma, USA & 94370 \\
\hline \multicolumn{4}{|l|}{ Enzymes } \\
\hline Benzonase* & & PEPC, MPI-CBG & - \\
\hline DNAse I & & NEB, USA & M0303 \\
\hline DpnI & & NEB, USA & $\mathrm{R} 0176$ \\
\hline Phusion HF & & NEB, USA & M0531 \\
\hline RNAseA & & Carl Roth, Germany & 7156.1 \\
\hline \multicolumn{4}{|l|}{ Ladders } \\
\hline 1kb DNA ladder & & NEB, USA & N3232 \\
\hline PageRuler Plus protein ladder & & Thermo, USA & 26616 \\
\hline Riboruler High Range RNA ladder & & Thermo, USA & SM1821 \\
\hline \multicolumn{4}{|l|}{ Dyes } \\
\hline $\begin{array}{l}\text { DFHBI ((Z)-4-(3,5-difluoro-4- } \\
\text { hydroxybenzylidene)-1,2-dimethyl-1H- } \\
\text { imidazol-5(4H)-one) }\end{array}$ & 252.22 & Sigma, USA & SML1627 \\
\hline $\begin{array}{l}\text { DiD (1,1'-Dioctadecyl-3,3,3',3'- } \\
\text { Tetramethylindodicarbocyanine, } 4 \text { - } \\
\text { Chlorobenzenesulfonate Salt) }\end{array}$ & 1052.08 & Invitrogen, USA & D7757 \\
\hline Gel Loading Dye & & NEB, USA & B7024 \\
\hline GelRed & & Biotium, USA & 41003 \\
\hline Laemmli buffer & & Bio-rad, USA & 1610737 \\
\hline SYBRgold & & Thermo, USA & S11494 \\
\hline
\end{tabular}




\begin{tabular}{|c|c|c|c|}
\hline $\begin{array}{l}\text { Texas Red DHPE (N-(Texas Red sulfonyl)- } \\
\text { 1,2-dihexadecanoyl-snglycero-3- } \\
\text { phosphoethanolamine, triethylammonium salt) }\end{array}$ & 1380.77 & Biotium, USA & 60027 \\
\hline \multicolumn{4}{|l|}{ Others } \\
\hline $1 \mathrm{H}, 1 \mathrm{H}, 2 \mathrm{H}, 2 \mathrm{H}$-perfluorodecyltrichlorosilane & 681.57 & Sigma, USA & 729965 \\
\hline 1-Octanol & 130.23 & Sigma, USA & 297887 \\
\hline $40 \%$ Polyacrylamide solution & - & Bio-rad, USA & 1610144 \\
\hline Agarose & - & Invitrogen, USA & 16500 \\
\hline Ammonium peroxydisulphate & 228.20 & Carl Roth, Germany & 9592.3 \\
\hline Ampicillin sodium salt & 371.39 & Sigma, USA & A9518 \\
\hline Protease Inhibitor Cocktail & - & Roche, Switzerland & COEDTAF-RO \\
\hline Dithiothreitol & 154.253 & Thermo, USA & R0862 \\
\hline DMSO & 78.13 & Sigma, USA & D8418 \\
\hline Egg PC (L- $\alpha$-phosphatidylcholine) & 770.123 & Avanti, USA & 840051 \\
\hline Folinic acid calcium salt hydrate & 511.50 & Sigma, USA & F7878 \\
\hline D-(+)-Glucose & 180.16 & Sigma, USA & G8270 \\
\hline Glycerol & 92.09 & VWR, USA & 24388.295 \\
\hline Gonotec calibration std. (300 mOsmol $/ \mathrm{kg}$ ) & - & Gonotec, Germany & 30.9 .0020 \\
\hline Gonotec calibration std. (2000 mOsmol/kg) & - & Gonotec, Germany & 30.9 .2000 \\
\hline $\begin{array}{l}\text { HEPES (N-2-Hydroxyethylpiperazine-N'-2- } \\
\text { ethane sulphonic acid) }\end{array}$ & 238.31 & Carl Roth, Germany & 9105 \\
\hline Hydrochloric acid fuming $37 \%$ & 36.46 & Merck, USA & 100317 \\
\hline Hydrogen peroxide & 34.01 & Sigma, USA & 16911 \\
\hline Imidazole & 68.08 & AppliChem & A 1073 \\
\hline Magnesium chloride hexahydrate & 203.30 & Sigma, USA & 105833 \\
\hline $\begin{array}{l}\text { L-Glutamic acid hemimagnesium salt } \\
\text { tetrahydrate }\end{array}$ & 388.61 & Sigma, USA & 49605 \\
\hline Mineral oil & $\begin{array}{l}(0.84 \mathrm{~g} / \mathrm{mL}) \\
(1.467 \mathrm{n} 20 / \mathrm{D})\end{array}$ & Sigma, USA & M5904 \\
\hline Nuclease-free water & - & Thermo, USA & AM9937 \\
\hline Pluronic acid F-68 & - & Gibco, USA & 24040032 \\
\hline Poly(4-styrenesulfonic acid) & $\sim 75000$ & Sigma, USA & 561223 \\
\hline Poly(diallyldimethylammonium chloride) & $\begin{array}{l}(1.04 \mathrm{~g} / \mathrm{mL}) \\
(1.375 \mathrm{n} 20 / \mathrm{D})\end{array}$ & Sigma, USA & 409014 \\
\hline $\begin{array}{l}\text { POPC (1-palmitoyl-2-oleoyl-glycero-3- } \\
\text { phosphocholine) }\end{array}$ & 760.076 & Avanti, USA & $850457 \mathrm{C}$ \\
\hline L-Glutamic acid potassium salt monohydrate & 203.23 & Sigma, USA & G1149 \\
\hline Potassium hydroxide & 56.11 & Sigma, USA & 221473 \\
\hline Potassium phosphate dibasic solution & 174.18 & Sigma, USA & P8584 \\
\hline Potassium phosphate monobasic solution & 136.086 & Sigma, USA & P8709 \\
\hline Sodium chloride & 58.44 & Merck, USA & 1.06404 \\
\hline Spermidine & 145.25 & Sigma, USA & S2626 \\
\hline Sucrose & 342.30 & Sigma, USA & S9378 \\
\hline SU-8 2025 & - & Microchem, USA & - \\
\hline $\begin{array}{l}\text { TEMED (N,N,N',N'- } \\
\text { Tetramethylethylenediamine) }\end{array}$ & 116.21 & Carl Roth, Germany & 2367.3 \\
\hline Trizma base & 121.14 & Sigma, USA & $\mathrm{T} 1503$ \\
\hline Tris-Glycine-SDS buffer (10X) & - & Bio-rad, USA & 1610772 \\
\hline Twinsil Speed silicone & - & Picodent, Germany & 13001002 \\
\hline Urea & 60.06 & Merck, USA & 108487 \\
\hline$\beta$-mercaptoethanol & 78.13 & Sigma, USA & M6250 \\
\hline
\end{tabular}

*Benzonase was prepared in-house by the Protein Expression, Purification, and Characterization (PEPC) facility, Max Planck Institute of Molecular Cell Biology and Genetics (MPI-CBG). 
Table S2. Kit list.

\begin{tabular}{lll}
\hline Name & Supplier & Catalog No. \\
\hline HiScribe T7 High Yield RNA Synthesis Kit & NEB, USA & E2040 \\
NEBuilder HiFi DNA Assembly Kit & NEB, USA & E2621 \\
PURExpress In Vitro Protein Synthesis Kit & NEB, USA & E6800 \\
QIAGEN Plasmid Maxi Kit & QIAGEN, Germany & 12162 \\
QIAGEN RNeasy Mini kit & QIAGEN, Germany & 74104 \\
QIAquick Gel Extraction Kit & QIAGEN, Germany & 28704 \\
QIAquick PCR Purification Kit & QIAGEN, Germany & 28104 \\
Quick Start Bradford Assay & Bio-Rad, USA & 5000201 \\
\hline
\end{tabular}

Table S3. Consumables list.

\begin{tabular}{lll}
\hline Name & Supplier & Catalog No. \\
\hline 24x60mm coverslip & Menzel-Gläser, Germany & - \\
384-well plates, Lobase, Black & Greiner Bio-One, Austria & 788096 \\
76x26x1mm microscope slides & Marienfeld, Germany & 1000000 \\
HisTrap FF column (5 ml) & Cytiva, USA & GE17-5255-01 \\
Millex syringe filter (PVDF) & Millipore, USA & SLGV033RS \\
Adhesive PCR Plate Seals & Thermo, USA & AB0558 \\
Spectra/Por 2 Dialysis Membrane (12-14 kD) & Repligen, USA & $132680 \mathrm{~T}$ \\
TGX Stain-Free PAGE Gel, 15-well, 4-15\% & Bio-Rad, USA & 4568086 \\
\hline
\end{tabular}

Table S4. Equipment list.

\begin{tabular}{lll}
\hline Name & Supplier & Catalog No. \\
\hline 100x/1.3 Oil Plan-Neofluar Ph3 M27 objective & Zeiss, Germany & $420491-9910-000$ \\
10X/0.45 Plan-Apochromat M27 objective & Zeiss, Germany & $420640-9900-000$ \\
40X/1.2 C-Apochromat W autocorr M27 objective & Zeiss, Germany & $421767-9971-790$ \\
5x/0.15 Plan-Neofluar Ph1 M27 objective & Zeiss, Germany & $420331-9911-000$ \\
Andor Axiovert 200M & Zeiss, Germany & - \\
Avanti Centrifuge J26-XP & Beckman Coulter, USA & - \\
Biovision Gel Doc system & Biovision, USA & - \\
Dimax S4 Monochrome sCMOS high-speed camera & PCO, Germany & - \\
JLA-8.1000 rotor & Beckman Coulter, USA & 363688 \\
LSM 880 with Airyscan & Zeiss, Germany & - \\
Mitos P-Pump & Dolomite, UK & 3200016 \\
NanoDrop 2000 & Thermo, USA & ND-2000 \\
Osmomat 3000 & Gonotec, Germany & - \\
pE-4000 illumination system & CoolLED, USA & - \\
Sonorex sonicator bath & Bandelin, Germany & - \\
Spark 20M plate reader & TECAN, Switzerland & - \\
Spin Coater WS-650MZ-23NPPB & Laurell Tech. Corp., USA & - \\
Typhoon 9500 Fluo and Phospho Imager & GE, USA & - \\
\hline
\end{tabular}




\section{Plasmid construction}

The strains and plasmids used in this study are listed in Table S5-6. Plasmids pEXP5-NT/6xHis eGFP [1] and pEXP5-NT/6xHis mCherry [2] were provided by JLR Anderson, University of Bristol. The pEXP5NT/6xHis mCherry F30-2xdBroccoli was made by inserting the F30-2xdBroccoli [3,4] downstream of the mCherry stop codon and upstream of the terminator of the mCherry gene. The F30-2xdBroccoli indicates two dimeric Broccoli RNA aptamers supported by an F30 stem loop. All plasmids have a high-copy number origin of replication. To create the insert for pEXP5-NT/6xHis mCherry F30-2xdBroccoli, Broccoli RNA aptamer inserts were synthesized as ultramers with overlapping regions to the pEXP5-NT/6xHis mCherry vector plasmid. F302xdBroccoli ultramers 1 and 2 were annealed, PCR amplified, and column purified (QIAquick PCR Purification Kit) to obtain the dsDNA insert. The pEXP5-NT/6xHis mCherry plasmid vector was divided into two parts by PCR amplifying the template plasmid pEXP5-NT/6xHis mCherry using primers NFB ptetO gBlock Fwd and mCherry Rev (vector part 1) and Tphi Fwd and DUR Vector 2 (vector part 2). The PCR products were then digested with DpnI to remove the plasmid template then gel purified (QIAquick Gel Extraction Kit) to obtain the vector fragments only. Plasmid assembly of the purified DNA parts for pEXP5-NT/6xHis mCherry F30-

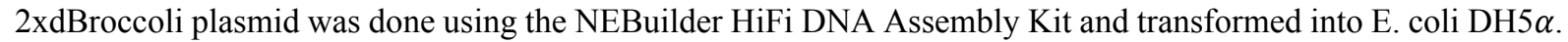
All plasmids were prepared and purified by ethanol precipitation from E. coli DH5 $\alpha$ cultures using the QIAGEN Plasmid Maxi Kit and measured by NanoDrop. Plasmid maps, sequences, and annotations are provided in Figs. S1-3, TableS S7-9, and Seqs. S1-3 (and in GenBank format in the supplementary files). Primer and ultramer sequences are provided in Tables S10-11. PCR cycling protocols are shown in Table S12. AGE results are provided in Fig. S4. All PCR, purification, and assembly methods were performed using the manufacturer's standard protocols. All primers and ultramers were synthesized by Integrated DNA Technologies (IDT, www.idtdna.com). Plasmid assembly was confirmed by Sanger sequencing (GENEWIZ, www.genewiz.com) using the primer pEXP5NT insert Rev. The pEXP5-NT/6xHis mCherry F30-2xdBroccoli plasmid is deposited in Addgene (www.addgene.org, plasmid ID 169233). Additionally, a plasmid with only one Broccoli aptamer downstream mCherry was also designed and deposited in Addgene (pEXP5-NT/6xHis mCherry F30-Broccoli, Addgene plasmid ID 169234).

Table S5. Strains used in this study.

\begin{tabular}{ccc}
\hline Strain name & \multicolumn{1}{c}{ Description } & Source \\
\hline E. coli DH5 $\alpha$ & High-efficiency competent cells for transformation. & NEB (C2987) \\
E. coli BL21 (DE3) & Competent cells for T7 RNAP-mediated expression. & NEB (C2527) \\
\hline
\end{tabular}

Table S6. Plasmids used in this study.

\begin{tabular}{ccc}
\hline Plasmid & Description & Source \\
\hline pEXP5-NT/6xHis eGFP & $\begin{array}{c}\text { Constitutive T7 RNAP-mediated expression } \\
\text { of 6xHis-eGFP. }\end{array}$ & {$[1]$} \\
pEXP5-NT/6xHis mCherry & $\begin{array}{c}\text { Constitutive T7 RNAP-mediated expression } \\
\text { of 6xHis-mCherry. }\end{array}$ & {$[2]$} \\
pEXP5-NT/6xHis mCherry & $\begin{array}{c}\text { Constitutive T7 RNAP-mediated expression } \\
\text { of 6xHis-mCherry with 2xdBroccoli RNA } \\
\text { aptamer. }\end{array}$ & This study. \\
\hline
\end{tabular}




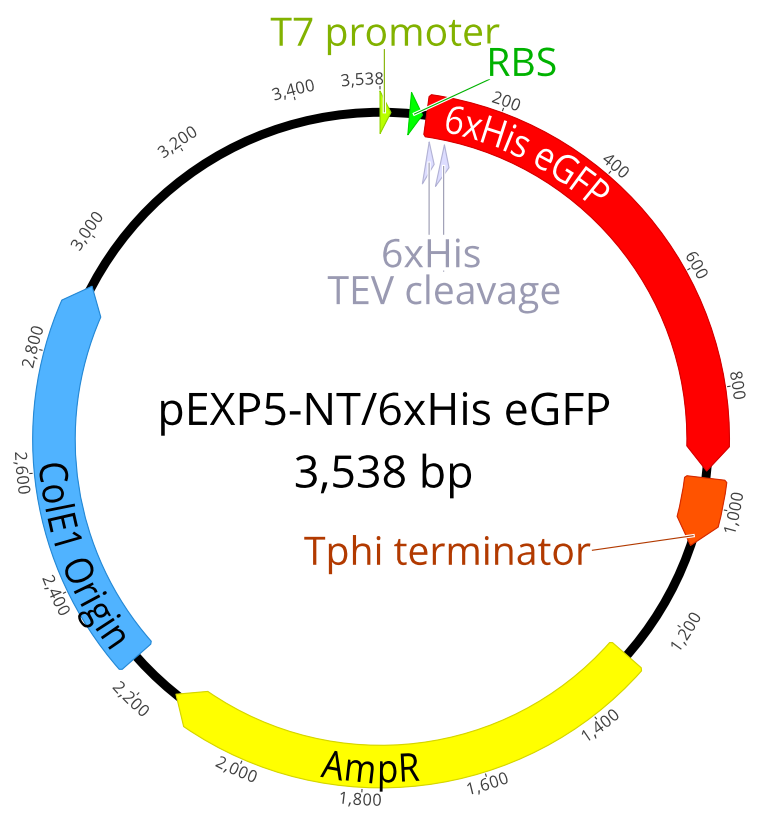

Figure S1. Plasmid map of pEXP5-NT/6xHis eGFP. The plasmid map illustration was created in Geneious 11.0.2 (www.geneious.com).

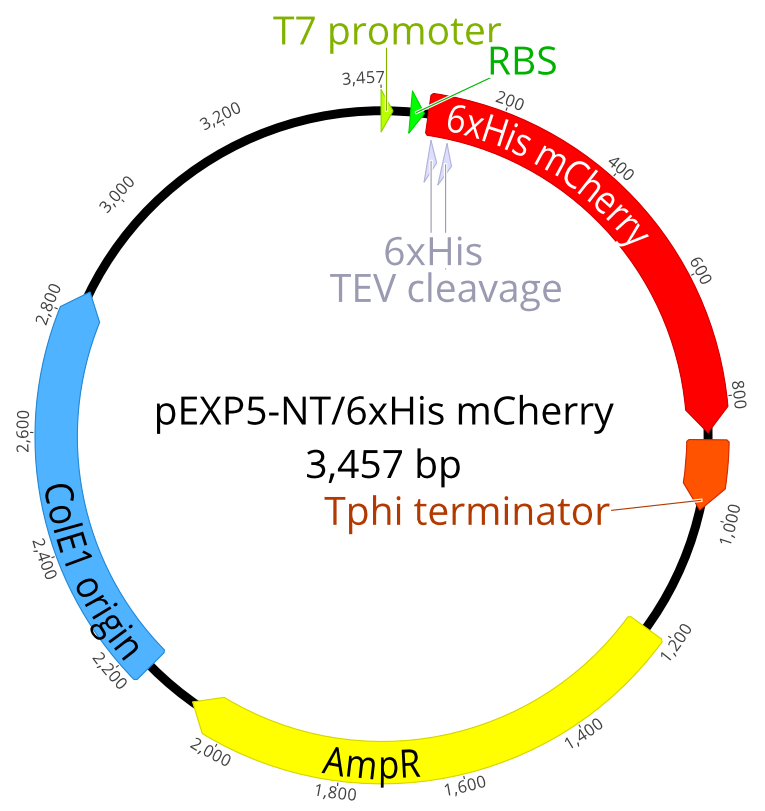

Figure S2. Plasmid map of pEXP5-NT/6xHis mCherry. The plasmid map illustration was created in Geneious 11.0.2 (www.geneious.com). 


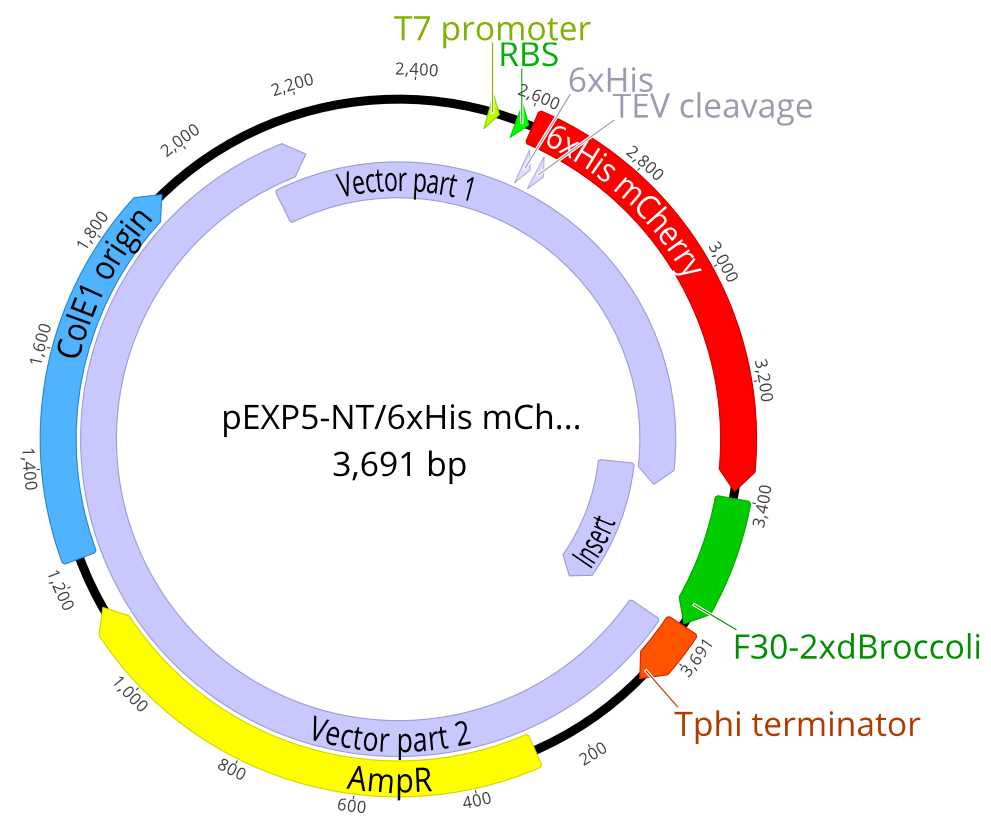

Figure S3. Plasmid map of pEXP5-NT/6xHis mCherry F30-2xdBroccoli. The DNA parts (vector parts 1-2 and insert) used to assemble the plasmid are annotated in light purple. The plasmid map illustration was created in Geneious 11.0.2 (www.geneious.com).

Table S7. Annotations of pEXP5-NT/6xHis eGFP plasmid.

\begin{tabular}{cccc}
\hline Annotation & Start & End & Length (bp) \\
\hline T7 promoter & 3538 & 18 & 19 \\
RBS & 50 & 72 & 23 \\
6xHis eGFP gene & 79 & 939 & 861 \\
6xHis & 91 & 108 & 18 \\
TEV cleavage & 121 & 138 & 18 \\
Tphi terminator & 952 & 1070 & 119 \\
AmpR gene & 1291 & 2151 & 861 \\
ColE1 Origin of replication & 2246 & 2928 & 683 \\
\hline
\end{tabular}

Table S8. Annotations of pEXP5-NT/6xHis mCherry plasmid.

\begin{tabular}{cccc}
\hline Annotation & Start & End & Length (bp) \\
\hline T7 promoter & 3,457 & 18 & 19 \\
RBS & 50 & 72 & 23 \\
6xHis mCherry gene & 79 & 858 & 780 \\
6xHis & 91 & 108 & 18 \\
TEV cleavage & 121 & 138 & 18 \\
Tphi terminator & 871 & 989 & 119 \\
AmpR gene & 1210 & 2070 & 861 \\
ColE1 Origin of replication & 2165 & 2847 & 683 \\
\hline
\end{tabular}

Table S9. Annotations of pEXP5-NT/6xHis mCherry F30-2xdBroccoli plasmid.

\begin{tabular}{cccc}
\hline Annotation & Start & End & Length (bp) \\
\hline T7 promoter & 2531 & 2549 & 19 \\
RBS & 2581 & 2603 & 23 \\
6xHis mCherry gene & 2610 & 3386 & 777 \\
6xHis & 2622 & 2639 & 18 \\
TEV cleavage & 2652 & 2669 & 18 \\
F30-2xdBroccoli aptamer & 3399 & 3632 & 234 \\
Tphi terminator & 3636 & 63 & 119 \\
AmpR gene & 284 & 1144 & 861 \\
ColE1 origin of replication & 1239 & 1921 & 683 \\
Vector part 1 & 2102 & 3397 & 1296 \\
F30-2xdBroccoli Insert & 3355 & 3691 & 337 \\
Vector part 2 & 3650 & 2187 & 2229 \\
\hline
\end{tabular}


Table S10. Primers used for cloning of the pEXP5-NT/6xHis mCherry F30-2xdBroccoli plasmid.

\begin{tabular}{ccc}
\hline Primer & Sequence (5'-3') & Purpose \\
\hline NFB ptetO gBlock Fwd & TTTCTCCTTACGCATCTGT & PCR of vector part 1 \\
mCherry Rev & ATCACCCTTTAGCTGCC & \\
Tphi Fwd & CGAAAGGAAGCTGAGTTG & PCR of vector part 2 \\
DUR vector 2 & CTGGCTTAACTATGCGGC & \\
pEXP5NT insert Rev & GGTTATTGTCTCATGAGCG & Sanger sequencing \\
\hline
\end{tabular}

Table S11. Ultramers for inserts of the pEXP5-NT/6xHis mCherry F30-2xdBroccoli plasmid.

\begin{tabular}{cc}
\hline Primer & Sequence (5'-3') \\
\hline F30-2xdBroccoli & CCGGCGGCATGGACGAGCTGTACAAGGGCAGCTAAAGGGTGATC \\
Ultramer 1 & TTGCCATGTGTATGTGGGAGACGGTCGGGTCCATCTGAGACGGTC \\
& GGGTCCAGATATTCGTATCTGTCGAGTAGAGTGTGGGCTCAGATG \\
& TCGAGTAGAGTGTGGGCTCCCACATACTCTGATGATCCAGACGGT \\
CGGGTCCATCTGA \\
F30-2xdBroccoli & TTATTGCTCAGCGGTGGCAGCAGCCAACTCAGCTTCCTTTCGGGC \\
Ultramer 2 & TTTGTTAGCAGCCGTTGCCATGAATGATCCAGCCCACACTCTACT \\
& CGACATCTGAGCCCACACTCTACTCGACAGATACGAATATCTGGA \\
& CCCGACCGTCTCAGATGGACCCGACCGTCTGGATCATCA \\
\hline
\end{tabular}

Table S12. PCR protocol for amplification of plasmid parts.

\begin{tabular}{ccc}
\hline PCR cycle & Temperature & Time \\
\hline Initial denaturation & $98^{\circ} \mathrm{C}$ & $40 \mathrm{secs}$ \\
Denaturation & $98^{\circ} \mathrm{C}$ & $10 \mathrm{secs}$ \\
Annealing & $53^{\circ} \mathrm{C}$ & $15 \mathrm{secs}$ \\
Extension & $72^{\circ} \mathrm{C}$ & $1.5 / 0.5 \mathrm{mins}$ \\
Final extension & $72^{\circ} \mathrm{C}$ & $5 \mathrm{mins}$ \\
Hold & $4^{\circ} \mathrm{C}$ & $\infty$ \\
\hline
\end{tabular}

*1.5 mins for vector parts $1-2$ and 0.5 mins for insert.

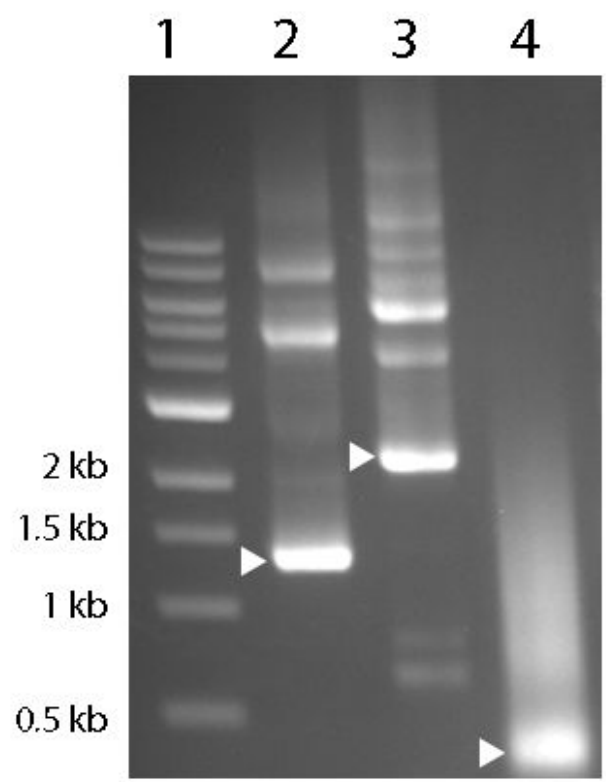

Figure S4. Agarose gel electrophoresis (1\% agarose, $120 \mathrm{~V}, 30$ mins, stained with GelRed) of DNA assembly parts for pEXP5-NT/6xHis mCherry F30-2xdBroccoli and pEXP5-NT/6xHis mCherry F30-Broccoli plasmids. Lane 1: $1 \mathrm{~kb}$ ladder, lane 2: PCR of vector part 1 (pT7-6xHis mCherry, $1296 \mathrm{bp}$ ), lane 3: PCR of vector part 2 (Tphi terminator-AmpR-ColE1 Ori, 2229 bp), and lane 4: PCR of insert (F30-2xdBroccoli, 337 bp. Target PCR products in lanes 2-4 are marked with a white arrow. Agarose gels were imaged using a Biovision Gel Doc system with $365 \mathrm{~nm}$ UV excitation. 
Sequence S1. Plasmid sequence of pEXP5-NT/6xHis eGFP.

AATACGACTCACTATAGGGAGACCACAACGGTTTCCCTCTAGAAATAATTTTGTTTAACTTTAAGA AGGAGATATACCATGTCTGGTTCTCATCATCATCATCATCATGGTAGCAGCGGCGAAAACCTGTAT TTTCAGTCCCTTGTGGGTACCGGTTCGAATGATGACGACGACAAGAGTCCGGATCCCAATTGGATG GTGAGCAAGGGCGAGGAGCTGTTCACCGGGGTGGTGCCCATCCTGGTCGAGCTGGACGGCGACGT AAACGGCCACAAGTTCAGCGTGTCCGGCGAGGGCGAGGGCGATGCCACCTACGGCAAGCTGACCC TGAAGTTCATCTGCACCACCGGCAAGCTGCCCGTGCCCTGGCCCACCCTCGTGACCACCCTGACCT ACGGCGTGCAGTGCTTCAGCCGCTACCCCGACCACATGAAGCAGCACGACTTCTTCAAGTCCGCCA TGCCCGAAGGCTACGTCCAGGAGCGCACCATCTTCTTCAAGGACGACGGCAACTACAAGACCCGC GCCGAGGTGAAGTTCGAGGGCGACACCCTGGTGAACCGCATCGAGCTGAAGGGCATCGACTTCAA GGAGGACGGCAACATCCTGGGGCACAAGCTGGAGTACAACTACAACAGCCACAACGTCTATATCA TGGCCGACAAGCAGAAGAACGGCATCAAGGTGAACTTCAAGATCCGCCACAACATCGAGGACGGC AGCGTGCAGCTCGCCGACCACTACCAGCAGAACACCCCCATCGGCGACGGCCCCGTGCTGCTGCCC GACAACCACTACCTGAGCACCCAGTCCGCCCTGAGCAAAGACCCCAACGAGAAGCGCGATCACAT GGTCCTGCTGGAGTTCGTGACCGCCGCCGGGATCACTCTCGGCATGGACGAGCTGTACAAGTCCGG ACTCAGATCTCGAGTCTGGTAAAGGGTGATCCGGCTGCTAACAAAGCCCGAAAGGAAGCTGAGTT GGCTGCTGCCACCGCTGAGCAATAACTAGCATAACCCCTTGGGGCCTCTAAACGGGTCTTGAGGGG TTTTTTGCTGAAAGGAGGAACTATATCCGGATAATTCTTGAAGACGAAAGGGCCTCGTGATACGCC TATTTTTATAGGTTAATGTCATGATAATAATGGTTTCTTAGACGTCAGGTGGCACTTTTCGGGGAAA TGTGCGCGGAACCCCTATTTGTTTATTTTTCTAAATACATTCAAATATGTATCCGCTCATGAGACAA TAACCCTGATAAATGCTTCAATAATATTGAAAAAGGAAGAGTATGAGTATTCAACATTTCCGTGTC GCCCTTATTCCCTTTTTTGCGGCATTTTGCCTTCCTGTTTTTGCTCACCCAGAAACGCTGGTGAAAGT AAAAGATGCTGAAGATCAGTTGGGTGCACGAGTGGGTTACATCGAACTGGATCTCAACAGCGGTA AGATCCTTGAGAGTTTTCGCCCCGAAGAACGTTTTCCAATGATGAGCACTTTTAAAGTTCTGCTATG TGGCGCGGTATTATCCCGTGTTGACGCCGGGCAAGAGCAACTCGGTCGCCGCATACACTATTCTCA GAATGACTTGGTTGAGTACTCACCAGTCACAGAAAAGCATCTTACGGATGGCATGACAGTAAGAG AATTATGCAGTGCTGCCATAACCATGAGTGATAACACTGCGGCCAACTTACTTCTGACAACGATCG GAGGACCGAAGGAGCTAACCGCTTTTTTGCACAACATGGGGGATCATGTAACTCGCCTTGATCGTT GGGAACCGGAGCTGAATGAAGCCATACCAAACGACGAGCGTGACACCACGATGCCTGTAGCAATG GCAACAACGTTGCGCAAACTATTAACTGGCGAACTACTTACTCTAGCTTCCCGGCAACAATTAATA GACTGGATGGAGGCGGATAAAGTTGCAGGACCACTTCTGCGCTCGGCCCTTCCGGCTGGCTGGTTT ATTGCTGATAAATCTGGAGCCGGTGAGCGTGGGTCTCGCGGTATCATTGCAGCACTGGGGCCAGAT GGTAAGCCCTCCCGTATCGTAGTTATCTACACGACGGGGAGTCAGGCAACTATGGATGAACGAAAT AGACAGATCGCTGAGATAGGTGCCTCACTGATTAAGCATTGGTAACTGTCAGACCAAGTTTACTCA TATATACTTTAGATTGATTTAAAACTTCATTTTTAATTTAAAAGGATCTAGGTGAAGATCCTTTTTG ATAATCTCATGACCAAAATCCCTTAACGTGAGTTTTCGTTCCACTGAGCGTCAGACCCCGTAGAAA AGATCAAAGGATCTTCTTGAGATCCTTTTTTTCTGCGCGTAATCTGCTGCTTGCAAACAAAAAAACC ACCGCTACCAGCGGTGGTTTGTTTGCCGGATCAAGAGCTACCAACTCTTTTTCCGAAGGTAACTGG CTTCAGCAGAGCGCAGATACCAAATACTGTTCTTCTAGTGTAGCCGTAGTTAGGCCACCACTTCAA GAACTCTGTAGCACCGCCTACATACCTCGCTCTGCTAATCCTGTTACCAGTGGCTGCTGCCAGTGGC GATAAGTCGTGTCTTACCGGGTTGGACTCAAGACGATAGTTACCGGATAAGGCGCAGCGGTCGGG CTGAACGGGGGGTTCGTGCACACAGCCCAGCTTGGAGCGAACGACCTACACCGAACTGAGATACC TACAGCGTGAGCTATGAGAAAGCGCCACGCTTCCCGAAGGGAGAAAGGCGGACAGGTATCCGGTA AGCGGCAGGGTCGGAACAGGAGAGCGCACGAGGGAGCTTCCAGGGGGAAACGCCTGGTATCTTTA TAGTCCTGTCGGGTTTCGCCACCTCTGACTTGAGCGTCGATTTTTGTGATGCTCGTCAGGGGGGCGG AGCCTATGGAAAAACGCCAGCAACGCGGCCTTTTTACGGTTCCTGGCCTTTTGCTGGCCTTTTGCTC ACATGTTCTTTCCTGCGTTATCCCCTGATTCTGTGGATAACCGTATTACCGCCTTTGAGTGAGCTGA TACCGCTCGCCGCAGCCGAACGACCGAGCGCAGCGAGTCAGTGAGCGAGGAAGCGGAAGAGCGC CTGATGCGGTATTTTCTCCTTACGCATCTGTGCGGTATTTCACACCGCAATGGTGCACTCTCAGTAC AATCTGCTCTGATGCCGCATAGTTAAGCCAGTATACACTCCGCTATCGCTACGCTTCGTTAATACAG ATGTAGGTGTTCCACAGGGTAGCCAGCAGCATCCTGCGATGCAGATCCGGAACATAATGGTGCAG GGCGCTGACTTCCGCGTTTCCAGACTTTACGAAACACGGAAACCGAAGACCATTCATGTTGTTGCT CAGGTCGCAGACGTTTTGCAGCAGCAGTCGCTTCACGTTCGCTCGCGTATCGGTGATTCATTCTGCT AACCAGTAAGGCAACCCCGCCAGCCTAGCCGGGTCCTCAACGACAGGAGCACGATCATGCGCACC CGTGGCCAGGACCCAACGCTGCCCGAGATCTCGATCCCGCGAAAT

Sequence S2. Plasmid sequence of pEXP5-NT/6xHis mCherry.

AATACGACTCACTATAGGGAGACCACAACGGTTTCCCTCTAGAAATAATTTTGTTTAACTTTAAGA AGGAGATATACCATGTCTGGTTCTCATCATCATCATCATCATGGTAGCAGCGGCGAAAACCTGTAT TTTCAGTCCATGGTGAGCAAGGGCGAGGAGGATAACATGGCCATCATCAAGGAGTTCATGCGCTTC AAGGTGCACATGGAGGGCTCCGTGAACGGCCACGAGTTCGAGATCGAGGGCGAGGGCGAGGGCC GCCCCTACGAGGGCACCCAGACCGCCAAGCTGAAGGTGACCAAGGGTGGCCCCCTGCCCTTCGCCT 
GGGACATCCTGTCCCCTCAGTTCATGTACGGCTCCAAGGCCTACGTGAAGCACCCCGCCGACATCC CCGACTACTTGAAGCTGTCCTTCCCCGAGGGCTTCAAGTGGGAGCGCGTGATGAACTTCGAGGACG GCGGCGTGGTGACCGTGACCCAGGACTCCTCCCTGCAGGACGGCGAGTTCATCTACAAGGTGAAG CTGCGCGGCACCAACTTCCCCTCCGACGGCCCCGTAATGCAGAAGAAGACCATGGGCTGGGAGGC CTCCTCCGAGCGGATGTACCCCGAGGACGGCGCCCTGAAGGGCGAGATCAAGCAGAGGCTGAAGC TGAAGGACGGCGGCCACTACGACGCTGAGGTCAAGACCACCTACAAGGCCAAGAAGCCCGTGCAG CTGCCCGGCGCCTACAACGTCAACATCAAGTTGGACATCACCTCCCACAACGAGGACTACACCATC GTGGAACAGTACGAACGCGCCGAGGGCCGCCACTCCACCGGCGGCATGGACGAGCTGTACAAGGG CAGCTAAAGGGTGATCCGGCTGCTAACAAAGCCCGAAAGGAAGCTGAGTTGGCTGCTGCCACCGC TGAGCAATAACTAGCATAACCCCTTGGGGCCTCTAAACGGGTCTTGAGGGGTTTTTTGCTGAAAGG AGGAACTATATCCGGATAATTCTTGAAGACGAAAGGGCCTCGTGATACGCCTATTTTTATAGGTTA ATGTCATGATAATAATGGTTTCTTAGACGTCAGGTGGCACTTTTCGGGGAAATGTGCGCGGAACCC CTATTTGTTTATTTTTCTAAATACATTCAAATATGTATCCGCTCATGAGACAATAACCCTGATAAAT GCTTCAATAATATTGAAAAAGGAAGAGTATGAGTATTCAACATTTCCGTGTCGCCCTTATTCCCTTT TTTGCGGCATTTTGCCTTCCTGTTTTTGCTCACCCAGAAACGCTGGTGAAAGTAAAAGATGCTGAAG ATCAGTTGGGTGCACGAGTGGGTTACATCGAACTGGATCTCAACAGCGGTAAGATCCTTGAGAGTT TTCGCCCCGAAGAACGTTTTCCAATGATGAGCACTTTTAAAGTTCTGCTATGTGGCGCGGTATTATC CCGTGTTGACGCCGGGCAAGAGCAACTCGGTCGCCGCATACACTATTCTCAGAATGACTTGGTTGA GTACTCACCAGTCACAGAAAAGCATCTTACGGATGGCATGACAGTAAGAGAATTATGCAGTGCTG CCATAACCATGAGTGATAACACTGCGGCCAACTTACTTCTGACAACGATCGGAGGACCGAAGGAG CTAACCGCTTTTTTGCACAACATGGGGGATCATGTAACTCGCCTTGATCGTTGGGAACCGGAGCTG AATGAAGCCATACCAAACGACGAGCGTGACACCACGATGCCTGTAGCAATGGCAACAACGTTGCG CAAACTATTAACTGGCGAACTACTTACTCTAGCTTCCCGGCAACAATTAATAGACTGGATGGAGGC GGATAAAGTTGCAGGACCACTTCTGCGCTCGGCCCTTCCGGCTGGCTGGTTTATTGCTGATAAATCT GGAGCCGGTGAGCGTGGGTCTCGCGGTATCATTGCAGCACTGGGGCCAGATGGTAAGCCCTCCCGT ATCGTAGTTATCTACACGACGGGGAGTCAGGCAACTATGGATGAACGAAATAGACAGATCGCTGA GATAGGTGCCTCACTGATTAAGCATTGGTAACTGTCAGACCAAGTTTACTCATATATACTTTAGATT GATTTAAAACTTCATTTTTAATTTAAAAGGATCTAGGTGAAGATCCTTTTTGATAATCTCATGACCA AAATCCCTTAACGTGAGTTTTCGTTCCACTGAGCGTCAGACCCCGTAGAAAAGATCAAAGGATCTT CTTGAGATCCTTTTTTTCTGCGCGTAATCTGCTGCTTGCAAACAAAAAAACCACCGCTACCAGCGGT GGTTTGTTTGCCGGATCAAGAGCTACCAACTCTTTTTCCGAAGGTAACTGGCTTCAGCAGAGCGCA GATACCAAATACTGTTCTTCTAGTGTAGCCGTAGTTAGGCCACCACTTCAAGAACTCTGTAGCACC GCCTACATACCTCGCTCTGCTAATCCTGTTACCAGTGGCTGCTGCCAGTGGCGATAAGTCGTGTCTT ACCGGGTTGGACTCAAGACGATAGTTACCGGATAAGGCGCAGCGGTCGGGCTGAACGGGGGGTTC GTGCACACAGCCCAGCTTGGAGCGAACGACCTACACCGAACTGAGATACCTACAGCGTGAGCTAT GAGAAAGCGCCACGCTTCCCGAAGGGAGAAAGGCGGACAGGTATCCGGTAAGCGGCAGGGTCGG AACAGGAGAGCGCACGAGGGAGCTTCCAGGGGGAAACGCCTGGTATCTTTATAGTCCTGTCGGGT TTCGCCACCTCTGACTTGAGCGTCGATTTTTGTGATGCTCGTCAGGGGGGCGGAGCCTATGGAAAA ACGCCAGCAACGCGGCCTTTTTACGGTTCCTGGCCTTTTGCTGGCCTTTTGCTCACATGTTCTTTCCT GCGTTATCCCCTGATTCTGTGGATAACCGTATTACCGCCTTTGAGTGAGCTGATACCGCTCGCCGCA GCCGAACGACCGAGCGCAGCGAGTCAGTGAGCGAGGAAGCGGAAGAGCGCCTGATGCGGTATTTT CTCCTTACGCATCTGTGCGGTATTTCACACCGCAATGGTGCACTCTCAGTACAATCTGCTCTGATGC CGCATAGTTAAGCCAGTATACACTCCGCTATCGCTACGCTTCGTTAATACAGATGTAGGTGTTCCAC AGGGTAGCCAGCAGCATCCTGCGATGCAGATCCGGAACATAATGGTGCAGGGCGCTGACTTCCGC GTTTCCAGACTTTACGAAACACGGAAACCGAAGACCATTCATGTTGTTGCTCAGGTCGCAGACGTT TTGCAGCAGCAGTCGCTTCACGTTCGCTCGCGTATCGGTGATTCATTCTGCTAACCAGTAAGGCAA CCCCGCCAGCCTAGCCGGGTCCTCAACGACAGGAGCACGATCATGCGCACCCGTGGCCAGGACCC AACGCTGCCCGAGATCTCGATCCCGCGAAAT

Sequence S3. Plasmid sequence of pEXP5-NT/6xHis mCherry F30-2xdBroccoli. CTAGCATAACCCCTTGGGGCCTCTAAACGGGTCTTGAGGGGTTTTTTGCTGAAAGGAGGAACTATA TCCGGATAATTCTTGAAGACGAAAGGGCCTCGTGATACGCCTATTTTTATAGGTTAATGTCATGATA ATAATGGTTTCTTAGACGTCAGGTGGCACTTTTCGGGGAAATGTGCGCGGAACCCCTATTTGTTTAT TTTTCTAAATACATTCAAATATGTATCCGCTCATGAGACAATAACCCTGATAAATGCTTCAATAATA TTGAAAAAGGAAGAGTATGAGTATTCAACATTTCCGTGTCGCCCTTATTCCCTTTTTTGCGGCATTT TGCCTTCCTGTTTTTGCTCACCCAGAAACGCTGGTGAAAGTAAAAGATGCTGAAGATCAGTTGGGT GCACGAGTGGGTTACATCGAACTGGATCTCAACAGCGGTAAGATCCTTGAGAGTTTTCGCCCCGAA GAACGTTTTCCAATGATGAGCACTTTTAAAGTTCTGCTATGTGGCGCGGTATTATCCCGTGTTGACG CCGGGCAAGAGCAACTCGGTCGCCGCATACACTATTCTCAGAATGACTTGGTTGAGTACTCACCAG TCACAGAAAAGCATCTTACGGATGGCATGACAGTAAGAGAATTATGCAGTGCTGCCATAACCATG AGTGATAACACTGCGGCCAACTTACTTCTGACAACGATCGGAGGACCGAAGGAGCTAACCGCTTTT TTGCACAACATGGGGGATCATGTAACTCGCCTTGATCGTTGGGAACCGGAGCTGAATGAAGCCATA 
CCAAACGACGAGCGTGACACCACGATGCCTGTAGCAATGGCAACAACGTTGCGCAAACTATTAAC TGGCGAACTACTTACTCTAGCTTCCCGGCAACAATTAATAGACTGGATGGAGGCGGATAAAGTTGC AGGACCACTTCTGCGCTCGGCCCTTCCGGCTGGCTGGTTTATTGCTGATAAATCTGGAGCCGGTGA GCGTGGGTCTCGCGGTATCATTGCAGCACTGGGGCCAGATGGTAAGCCCTCCCGTATCGTAGTTAT CTACACGACGGGGAGTCAGGCAACTATGGATGAACGAAATAGACAGATCGCTGAGATAGGTGCCT CACTGATTAAGCATTGGTAACTGTCAGACCAAGTTTACTCATATATACTTTAGATTGATTTAAAACT TCATTTTTAATTTAAAAGGATCTAGGTGAAGATCCTTTTTGATAATCTCATGACCAAAATCCCTTAA CGTGAGTTTTCGTTCCACTGAGCGTCAGACCCCGTAGAAAAGATCAAAGGATCTTCTTGAGATCCT TTTTTTCTGCGCGTAATCTGCTGCTTGCAAACAAAAAAACCACCGCTACCAGCGGTGGTTTGTTTGC CGGATCAAGAGCTACCAACTCTTTTTCCGAAGGTAACTGGCTTCAGCAGAGCGCAGATACCAAATA CTGTTCTTCTAGTGTAGCCGTAGTTAGGCCACCACTTCAAGAACTCTGTAGCACCGCCTACATACCT CGCTCTGCTAATCCTGTTACCAGTGGCTGCTGCCAGTGGCGATAAGTCGTGTCTTACCGGGTTGGAC TCAAGACGATAGTTACCGGATAAGGCGCAGCGGTCGGGCTGAACGGGGGGTTCGTGCACACAGCC CAGCTTGGAGCGAACGACCTACACCGAACTGAGATACCTACAGCGTGAGCTATGAGAAAGCGCCA CGCTTCCCGAAGGGAGAAAGGCGGACAGGTATCCGGTAAGCGGCAGGGTCGGAACAGGAGAGCG CACGAGGGAGCTTCCAGGGGGAAACGCCTGGTATCTTTATAGTCCTGTCGGGTTTCGCCACCTCTG ACTTGAGCGTCGATTTTTGTGATGCTCGTCAGGGGGGCGGAGCCTATGGAAAAACGCCAGCAACGC GGCCTTTTTACGGTTCCTGGCCTTTTGCTGGCCTTTTGCTCACATGTTCTTTCCTGCGTTATCCCCTG ATTCTGTGGATAACCGTATTACCGCCTTTGAGTGAGCTGATACCGCTCGCCGCAGCCGAACGACCG AGCGCAGCGAGTCAGTGAGCGAGGAAGCGGAAGAGCGCCTGATGCGGTATTTTCTCCTTACGCAT CTGTGCGGTATTTCACACCGCAATGGTGCACTCTCAGTACAATCTGCTCTGATGCCGCATAGTTAAG CCAGTATACACTCCGCTATCGCTACGCTTCGTTAATACAGATGTAGGTGTTCCACAGGGTAGCCAG CAGCATCCTGCGATGCAGATCCGGAACATAATGGTGCAGGGCGCTGACTTCCGCGTTTCCAGACTT TACGAAACACGGAAACCGAAGACCATTCATGTTGTTGCTCAGGTCGCAGACGTTTTGCAGCAGCAG TCGCTTCACGTTCGCTCGCGTATCGGTGATTCATTCTGCTAACCAGTAAGGCAACCCCGCCAGCCTA GCCGGGTCCTCAACGACAGGAGCACGATCATGCGCACCCGTGGCCAGGACCCAACGCTGCCCGAG ATCTCGATCCCGCGAAATAATACGACTCACTATAGGGAGACCACAACGGTTTCCCTCTAGAAATAA TTTTGTTTAACTTTAAGAAGGAGATATACCATGTCTGGTTCTCATCATCATCATCATCATGGTAGCA GCGGCGAAAACCTGTATTTTCAGTCCATGGTGAGCAAGGGCGAGGAGGATAACATGGCCATCATC AAGGAGTTCATGCGCTTCAAGGTGCACATGGAGGGCTCCGTGAACGGCCACGAGTTCGAGATCGA GGGCGAGGGCGAGGGCCGCCCCTACGAGGGCACCCAGACCGCCAAGCTGAAGGTGACCAAGGGT GGCCCCCTGCCCTTCGCCTGGGACATCCTGTCCCCTCAGTTCATGTACGGCTCCAAGGCCTACGTGA AGCACCCCGCCGACATCCCCGACTACTTGAAGCTGTCCTTCCCCGAGGGCTTCAAGTGGGAGCGCG TGATGAACTTCGAGGACGGCGGCGTGGTGACCGTGACCCAGGACTCCTCCCTGCAGGACGGCGAG TTCATCTACAAGGTGAAGCTGCGCGGCACCAACTTCCCCTCCGACGGCCCCGTAATGCAGAAGAAG ACCATGGGCTGGGAGGCCTCCTCCGAGCGGATGTACCCCGAGGACGGCGCCCTGAAGGGCGAGAT CAAGCAGAGGCTGAAGCTGAAGGACGGCGGCCACTACGACGCTGAGGTCAAGACCACCTACAAGG CCAAGAAGCCCGTGCAGCTGCCCGGCGCCTACAACGTCAACATCAAGTTGGACATCACCTCCCACA ACGAGGACTACACCATCGTGGAACAGTACGAACGCGCCGAGGGCCGCCACTCCACCGGCGGCATG GACGAGCTGTACAAGGGCAGCTAAAGGGTGATCTTGCCATGTGTATGTGGGAGACGGTCGGGTCC ATCTGAGACGGTCGGGTCCAGATATTCGTATCTGTCGAGTAGAGTGTGGGCTCAGATGTCGAGTAG AGTGTGGGCTCCCACATACTCTGATGATCCAGACGGTCGGGTCCATCTGAGACGGTCGGGTCCAGA TATTCGTATCTGTCGAGTAGAGTGTGGGCTCAGATGTCGAGTAGAGTGTGGGCTGGATCATTCATG GCAACGGCTGCTAACAAAGCCCGAAAGGAAGCTGAGTTGGCTGCTGCCACCGCTGAGCAATAA 


\section{3. mRNA expression and purification}

The mRNA transcript of the pEXP5-NT/6xHis mCherry F30-2xdBroccoli plasmid containing the mCherry gene and Broccoli RNA aptamer was transcribed in vitro and purified for RNA calibration and RNA titration experiments. To prepare the DNA template for in vitro transcription (IVT), the region containing the T7 promoter up to the terminator of the pEXP5-NT/6xHis mCherry F30-2xdBroccoli plasmid was PCR amplified (Phusion HF) using primers pEXP5-NT Insert Fwd and Rev (Tables S13-14), digested with DpnI (NEB, 20 units/50ul PCR reaction volume) to remove the plasmid template, and purified (QIAquick PCR Purification Kit) (Fig. S5A). The purified PCR product was used as the template for IVT using the HiScribe T7 High Yield RNA Synthesis Kit which was used according to manufacturer's instructions with 5 hours incubation at $37{ }^{\circ} \mathrm{C}$ and DNAse I treatment. The IVT reaction product is then purified using the QIAGEN RNeasy kit as described by the manufacturer. The purified mRNA was confirmed by Nanodrop 260/280 ratios of $\sim 2.0$, native agarose gel, and denaturing urea-PAGE (Fig. S5B-C). We observed two separate bands in the denaturing urea polyacrylamide gel for the 6xHis mCherry F30-2xdBroccoli RNA transcript. To check if these two bands still contain the Broccoli aptamer, an in-gel DFHBI staining was performed according to [4], which showed that both bands fluoresce with DFHBI (Fig S5D). Therefore, both fractions had the complete RNA aptamer sequences. The two bands in the denaturing RNA gel could be a result of transcription read-through over the terminator of the PCR amplicon DNA template. However, we assumed that both species contained the F30-2xdBroccoli RNA aptamer because the DNA template is from a pure single band PCR product, both RNA bands are only slightly longer than the expected size $(\sim 1224 \mathrm{bp})$, and both bands are fluorescent with the DFHBI from the in-gel staining. Therefore, using this purified mRNA for our calibration curves is consistent on a molar concentration basis. Purified mRNA was stored at -20 ${ }^{\circ} \mathrm{C}$ until further use.

Table S13. Primers used to amplify IVT DNA template.

\begin{tabular}{cc}
\hline Primer & Sequence (5'-3') \\
\hline pEXP5-NT Insert Fwd & TCGGTGATTCATTCTGCT \\
pEXP5-NT Insert Rev & GGTTATTGTCTCATGAGCG \\
\hline
\end{tabular}

Table S14. PCR protocol for amplification of IVT DNA template.

\begin{tabular}{ccc}
\hline PCR cycle & Temperature & Time \\
\hline Initial denaturation & $98^{\circ} \mathrm{C}$ & $40 \mathrm{secs}$ \\
Denaturation & $98^{\circ} \mathrm{C}$ & $10 \mathrm{secs}$ \\
Annealing & $53^{\circ} \mathrm{C}$ & $15 \mathrm{secs}$ \\
Extension & $72^{\circ} \mathrm{C}$ & $45 \mathrm{secs}$ \\
Final extension & $72^{\circ} \mathrm{C}$ & $5 \mathrm{mins}$ \\
Hold & $10^{\circ} \mathrm{C}$ & $\infty$ \\
\hline
\end{tabular}
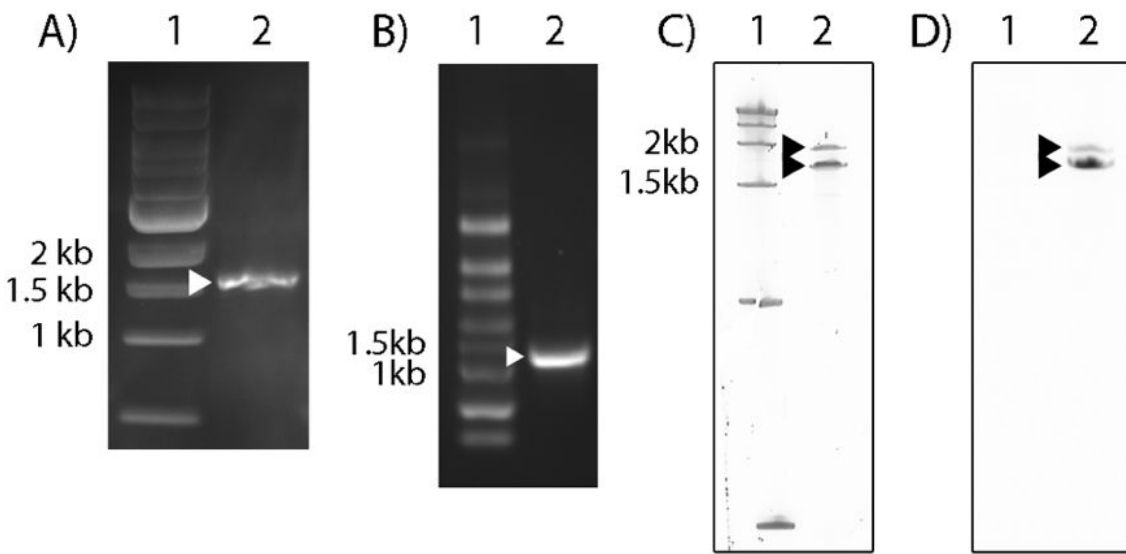

Figure S5. In vitro transcription and purification of mRNA. (A) AGE of DNA template for IVT reaction amplified from pEXP5-NT/6xHis mCherry F30-2xdBroccoli plasmid using primers pEXP5NT Insert Fwd and Rev (2\% agarose, $150 \mathrm{~V}, 30$ mins, stained with GelRed). Lane 1: $1 \mathrm{~kb}$ ladder, lane 2: PCR product (1534 bp). (B) Purified RNA from IVT reaction in a native agarose gel (1\% agarose, $120 \mathrm{~V}, 40$ mins, stained with GelRed). Lane 1: Riboruler High Range RNA ladder, lanes 2-4: dilutions of purified RNA transcript (expected size $\sim 1224 \mathrm{bp}$ ). (C) Purified RNA from IVT reaction in a denaturing urea polyacrylamide gel (3\% acrylamide, $8 \mathrm{M}$ urea, $200 \mathrm{~V}, 45$ mins, stained with SYBRgold). Lane 1: Riboruler High Range RNA ladder, lane 2: purified RNA transcript showing two separate bands (black arrows). (D) In-gel staining of purified RNA from IVT reaction in a denaturing urea polyacrylamide gel. After running the gel (3\% acrylamide, $8 \mathrm{M}$ urea, $200 \mathrm{~V}, 45 \mathrm{mins}$ ), the gel was washed 
$3 \mathrm{x}$ with water for 5 minutes and then stained with $10 \mu \mathrm{M}$ DFHBI, $40 \mathrm{mM}$ HEPES (pH 7.4), $100 \mathrm{mM} \mathrm{KCl}$, and 1 $\mathrm{mM} \mathrm{MgCl} 2$ for 30 mins before imaging. Lane 1: Riboruler High Range RNA ladder, lane 2: purified RNA transcript showing two DFHBI stained bands (black arrows). Agarose gels were imaged using a Biovision Gel Doc system with $365 \mathrm{~nm}$ UV excitation. Polyacrylamide gels were imaged using a Typhoon 9500 Fluo and Phospho Imager (BPB1-530DF20 filter). 


\section{Protein expression and purification}

pEXP5-NT/6xHis eGFP and pEXP5-NT/6xHis mCherry plasmids were transformed into E. coli BL21 (DE3) strains and used for protein expression and purification of eGFP and mCherry 6xHis-tagged proteins for quantitative calibration. All culture media was composed of LB with $100 \mu \mathrm{g} / \mathrm{ml}$ Ampicillin. For both mCherry and eGFP protein expression, overnight cultures were used to inoculate $500 \mathrm{ml}$ of production cultures with $40 \mu \mathrm{M}$ IPTG to a starting $\mathrm{OD}_{600}$ of 0.015 . After incubation at $37^{\circ} \mathrm{C}$ and stirring at $180 \mathrm{rpm}$ for approximately 4 hours $\left(\mathrm{OD}_{600} \sim 5\right)$, the cultures were centrifuged at $5000 \mathrm{rpm}$ (Avanti Centrifuge J26-XP with JLA-8.1000 rotor, Beckman Coulter) for 5 minutes at $4{ }^{\circ} \mathrm{C}$ to pellet the cells. The media was removed and the cells resuspended in lysis buffer $(50 \mathrm{~mL}$ lysis buffer/L culture) (Table S15). The resuspended cells were lysed by high-pressure homogenization by running the cell dispersion through the Emulsiflex C5-Avestin at 1500 bar two times. The lysed extracts were then clarified by centrifugation at $16000 \mathrm{rpm}$ for 30 minutes and filtered through a $0.45 \mu \mathrm{m}$ membrane (Millex-HV Low Protein Binding Durapore Membrane (PVDF), $33 \mathrm{~mm}$ diameter filter unit, $0.45 \mu \mathrm{m}$ pore size) to collect only the soluble protein fraction. Ni-Sepharose resin HisTrap columns (HisTrap FF $5 \mathrm{ml}$, Cytiva) were washed with water and preequilibriated with $25 \mathrm{ml}$ of the same lysis buffer. The clarified extracts containing the His-tagged proteins were loaded on the column, washed with $25 \mathrm{ml}$ lysis buffer, $25 \mathrm{ml}$ high salt buffer, and $25 \mathrm{ml}$ lysis buffer at a rate of 5 $\mathrm{ml} / \mathrm{min}$. The proteins were eluted from the column with elution buffer (Table S15) at $2 \mathrm{ml} / \mathrm{min}$. The protein was collected at 18 fractions at $2 \mathrm{ml}$ each. A Bradford assay (Bio-Rad) was run on each of the 18 fractions to determine which fraction from the column contained the His-tagged protein to collect. The selected fractions were placed in a 12-14 kDa cut-off dialysis membrane (Spectra/Por 2 Dialysis Membrane Standard RC Tubing MWCO: 12-14 $\mathrm{kDa}$ ) and dialyzed at $4{ }^{\circ} \mathrm{C}$ overnight in storage buffer (Table S15). After dialysis, aliquots of the purified proteins were flash frozen in liquid nitrogen and stored at $-80^{\circ} \mathrm{C}$ until use. All media and buffer recipes are listed in Table S15. Purified proteins were confirmed by size using SDS or Native PAGE (Bio-Rad TGX Stain-Free PAGE Gel, 15-well, 4-15\%) and absorbance spectra using UV-vis scanning (Nanodrop) (Fig. S6). To load the gels, $5 \mu 1$ of sample was mixed with $5 \mu \mathrm{l}$ of $2 \mathrm{X}$ Laemmli buffer, incubated at $95{ }^{\circ} \mathrm{C}$ for 5 mins, and then $7 \mu l$ was loaded onto the gel (Bio-Rad TGX Stain-Free PAGE Gel, 15-well, 4-15\%). Samples were run at $200 \mathrm{~V}$ for 30 mins in $1 X$ TrisGlycine-sodium dodecyl sulfate (SDS) or 1X Tris-Glycine buffer.

Table S15. Media and buffer recipes for protein expression and purification.

\begin{tabular}{lll}
\hline Buffer & Components & Concentration \\
\hline LB media & $\mathrm{NaCl}$ & $10 \mathrm{~g} / \mathrm{L}$ \\
& Tryptone & $10 \mathrm{~g} / \mathrm{L}$ \\
& Yeast extract & $5 \mathrm{~g} / \mathrm{L}$ \\
& Ampicillin & $100 \mu \mathrm{g} / \mathrm{ml}$ \\
\hline Lysis buffer & PBS & $2 \mathrm{X}$ \\
& Imidazole & $20 \mathrm{mM}$ \\
& DTT & $1 \mathrm{mM}$ \\
& Benzonase & $0.025 \mathrm{mg} / \mathrm{L}$ \\
& Protease inhibitor & 1 tablet/L \\
& $\mathrm{MgCl}{ }_{2}$ & $1 \mathrm{mM}$ \\
& $\mathrm{pH}(+\mathrm{HCl} / \mathrm{NaOH})$ & 7.5 \\
\hline HiSalt buffer & $\mathrm{PBS}$ & $6 \mathrm{X}$ \\
& $\mathrm{pH}(+\mathrm{HCl} / \mathrm{NaOH})$ & 7.5 \\
\hline Elution buffer & $\mathrm{PBS}$ & $2 \mathrm{X}$ \\
& $\mathrm{DTT}$ & $0.3 \mathrm{mM}$ \\
& $\mathrm{Imidazole}$ & $250 \mathrm{mM}$ \\
\hline Storage buffer & TrisHCl & $25 \mathrm{mM}$ \\
& $\mathrm{NaCl}$ & $150 \mathrm{mM}$ \\
& $\mathrm{Glycerol}$ & $5 \%$ \\
& $\mathrm{pH}(+\mathrm{HCl} / \mathrm{NaOH})$ & 8 \\
\hline 1X PBS buffer & $\mathrm{NaCl}$ & $137 \mathrm{mM}$ \\
& $\mathrm{KCl}$ & $2.7 \mathrm{mM}$ \\
& $\mathrm{Na} \mathrm{HPO}_{4}$ & $10 \mathrm{mM}$ \\
& $\mathrm{KH} \mathrm{PO}_{4}$ & $2 \mathrm{mM}$ \\
& $\mathrm{pH}(+\mathrm{HCl} / \mathrm{NaOH})$ & \\
& & 7.5 \\
\hline
\end{tabular}



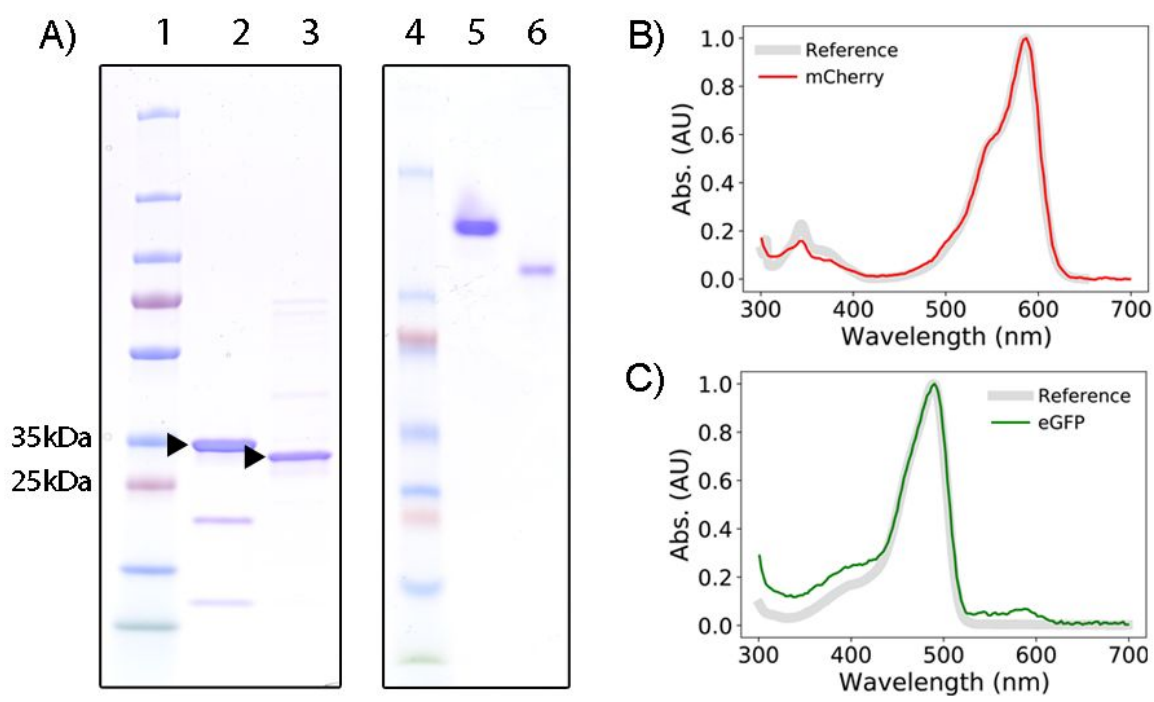

Figure S6. Purified mCherry and eGFP proteins. (A) SDS PAGE of purified proteins. Lane 1 - PageRuler Plus protein ladder (Thermo, USA), lane 2 - purified mCherry (29.222 kDa), and lane 3 - purified eGFP (32.153 kDa). Low molecular weight bands $(\sim 20 \mathrm{kDa}$ and $\sim 12 \mathrm{kDa})$ visible in the mCherry lanes are self-cleaved products of mCherry as a result of sample boiling prior to SDS PAGE [5]. Target protein products in lanes 2 and 3 are marked with a black arrow. Lanes 4-6 are native non-denaturing gels of the same samples that show single bands of the proteins. Lane 1 - PageRuler Plus protein ladder (Thermo, USA), lane 2 - purified mCherry (29.222 kDa), and lane 3 - purified eGFP (32.153 kDa). Purified 6xHis mCherry (B) and 6xHis eGFP (C) absorbance spectra compared to its reference spectra (www.fpbase.org/protein/egfp/ and www.fpbase.org/protein/mCherry/). 


\section{Protein and mRNA calibration}

To obtain calibration curved for eGFP and mCherry, purified stock solutions of eGFP and mCherry proteins were quantified by Bradford assay (Quick Start Bradford Assay, Bio-Rad) with a BSA protein standard and NanoDrop A280 readings. The purified 6xHis mCherry F30-2xdBroccoli mRNA stock solution was quantified using NanoDrop RNA-40 readings. Mean values obtained from the Bradford assay were used for downstream calculations for protein concentrations and calibration (Table S16). Serial dilutions of protein and mRNA stock solutions were prepared with a PURExpress master mix solution (Table S18) without plasmid DNA or mRNA, with and without DFHBI to ensure equivalent conditions between the gene expression reaction mixture and the calibration samples required for correct calibrations. These calibration standards were then loaded in a 384-well plate $(12.5 \mu \mathrm{l} /$ well $)$ or loaded on a glass slide $(2 \mu \mathrm{l} / \mathrm{spot})$ (Fig. S7). Glass slides comprised of a parafilm border sandwiched by a glass slide $(76 \times 26 \times 1 \mathrm{~mm}$ microscope slides, Marienfeld) and coverslip (24x60 mm, MenzelGläser). Linear calibration curves to convert relative fluorescence units (RFU) to concentration units (nM) were obtained for eGFP, mCherry, and mRNA F30-2xdBroccoli aptamer in both the plate reader (Figs. S8-9) and confocal microscopy (Figs. S10-11) at the same acquisition settings for bulk and liposome-encapsulated experiments. Histograms of RFU per pixel in the ROIs of the calibration standards (Figs. S8B and S9B) show increase of fluorescence distributions as a function of increasing standard concentration. Fluorescence sampling uses the mean of the circular ROIs in Fig. S36 across the microscopy images and calculates standard deviations from these mean ROI values (Figs. S8A and S9A). Higher laser intensities were avoided to prevent bleaching effects of the CFES. Our methodologies provide an accurate method for quantification of mRNA and protein concentrations within the synthetic cells from confocal imaging. It should be noted that we used an 8-bit format for our image acquisition settings, which provides RFU values ranging from $0-256$. In this instance, this imaging format was sufficient to provide enough differentiation for calibration of different standard concentrations. If better differentiation was required between signal values then a 16-bit imaging format can be used to provide finer range RFU values from 0 to 65535. Excitation/emission wavelengths in the TECAN Spark 20M plate reader were $485 / 535 \mathrm{~nm}$ (Gain 50), 570/620 nm (Gain 70), and 450/510 nm (Gain 60) with a bandwidth of $\pm 20 \mathrm{~nm}$ each, for eGFP, mCherry, and Broccoli RNA, respectively. For confocal microscopy (LSM 880 with Airyscan, 10X/0.45 Plan-Apochromat M27 objective), excitation/detection wavelengths are $488 \mathrm{~nm} / 499-561 \mathrm{~nm}$ for RNA Broccoli and eGFP protein, and $561 \mathrm{~nm} / 579-641 \mathrm{~nm}$ for mCherry protein. As the $488 \mathrm{~nm}$ laser power gradually decreased between experiments, the mRNA F30-2xdBroccoli aptamer calibration curve used for DNA titration in synthetic cell populations experiment was interpolated between two calibration curves based on the measured laser power (Fig. S10A). Image analysis and collection of the RFU values from confocal images for calibration are further discussed in Section 13.

Table S16. eGFP protein, mCherry protein, and 6xHis mCherry F30-2xdBroccoli mRNA stock concentrations. Protein concentrations were measured by Bradford assay and A280 measurements on the Nanodrop (in parentheses). mRNA concentration was measured by NanoDrop RNA-40 readings.

\begin{tabular}{cccc}
\hline Stock & $\boldsymbol{\mu M}$ & $\mathbf{A 2 6 0 / 2 8 0}$ & $\mathbf{A 2 6 0 / 2 3 0}$ \\
\hline eGFP protein & $79.34 \pm 9.47(79.93)$ & - & - \\
mCherry protein & $226.74 \pm 10.63(284.72)$ & - & - \\
6xHis mCherry F30-2xdBroccoli mRNA & 26.42 & 2 & 2.34 \\
\hline
\end{tabular}

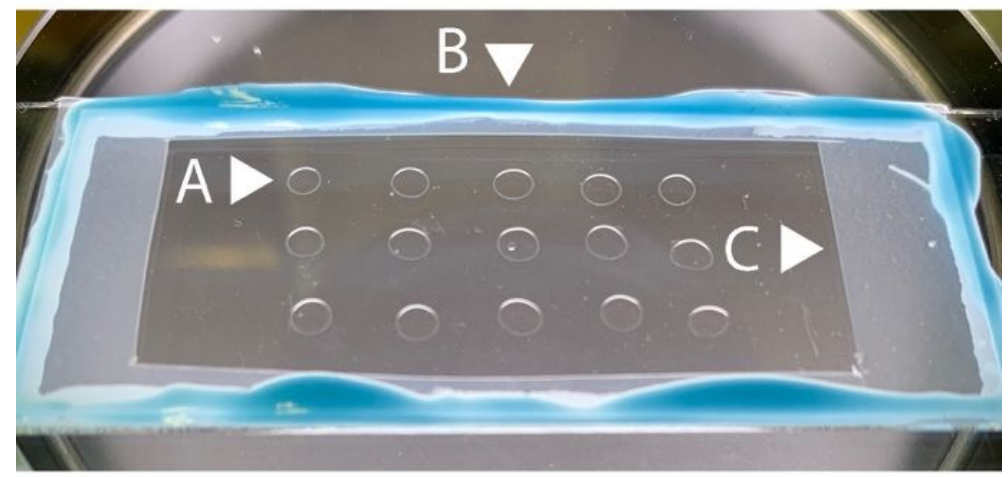

Figure S7. Glass slide setup for confocal microscopy calibration standards. (A) $2 \mu 1$ spots of standard solutions for calibration. (B) Twinsil Speed silicone sealing the glass slide and cover slip setup. (C) Parafilm layer between glass slide and coverslip. 

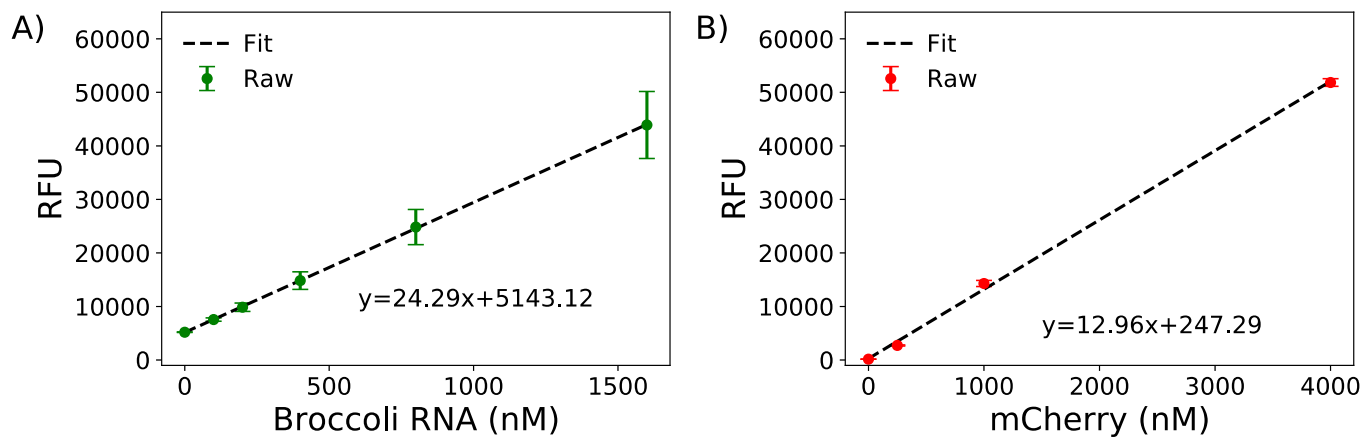

Figure S8. Linear calibration curve of (A) F30-2xdBroccoli RNA aptamer and (B) mCherry protein in PURExpress master mix with $10 \mu \mathrm{M}$ DFHBI in a bulk plate reader format. Mean and standard deviation bars are calculated from triplicate samples.
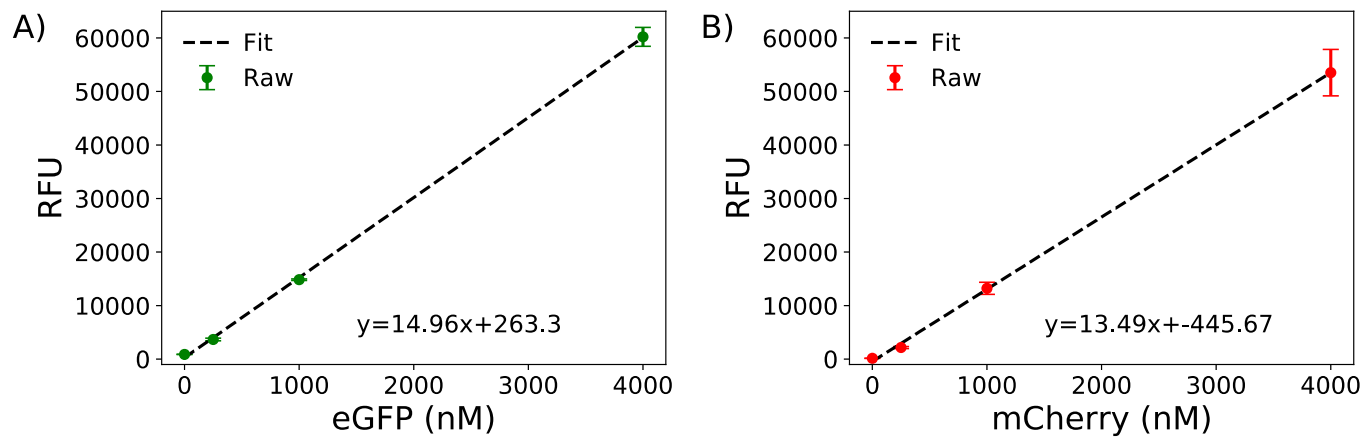

Figure S9. Linear calibration curve of (A) eGFP protein and (B) mCherry protein in PURExpress mastermix without DFHBI in a bulk plate reader format. Mean and standard deviation bars are calculated from triplicate samples.

A)
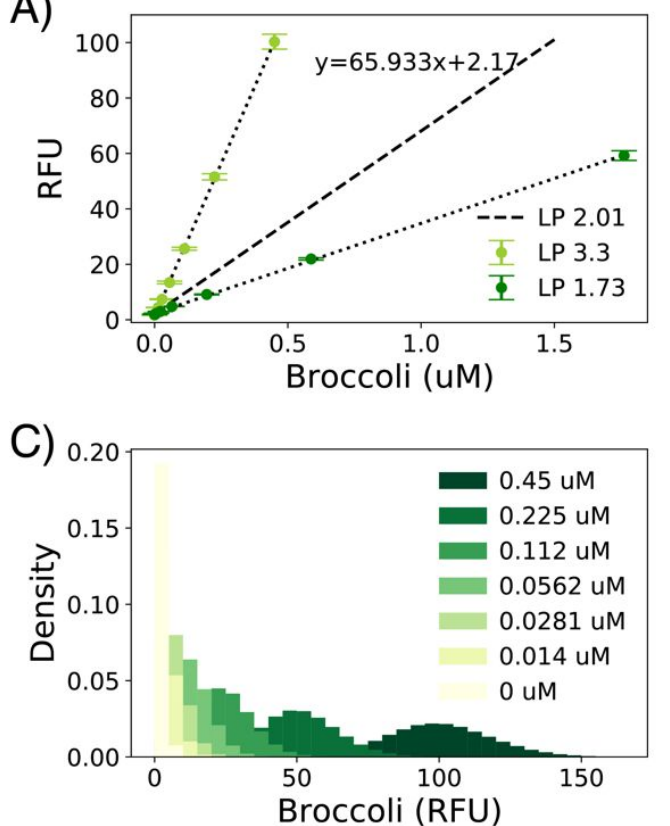

B)

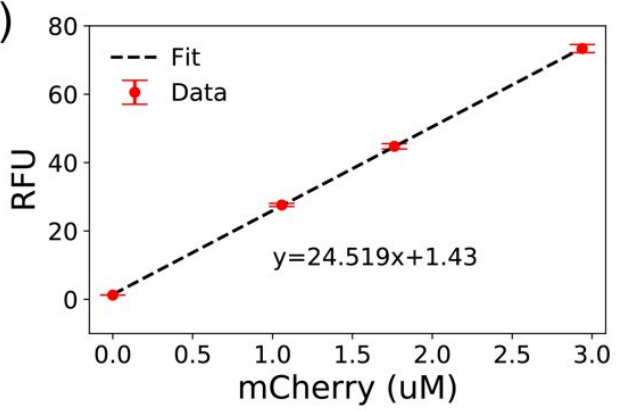

D)

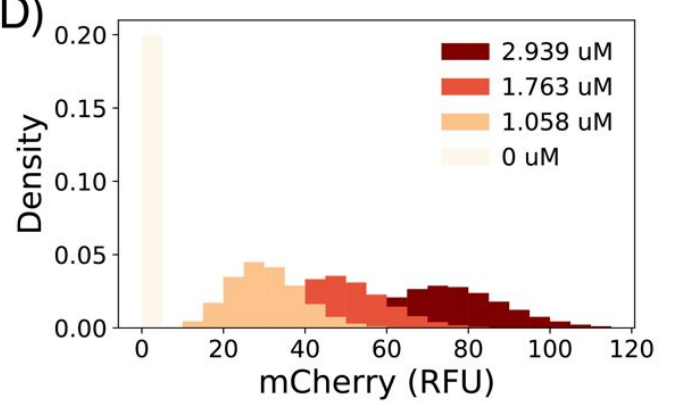

Figure S10. Calibration curves of (A) F30-2xdBroccoli RNA aptamer and (B) mCherry protein in PURExpress master mix with $10 \mathrm{uM}$ DFHBI obtained from confocal images. Dotted lines in (A) are F30-2xdBroccoli RNA aptamer calibration curves at laser powers $3.3 \mathrm{~mW}$ (light green) and $1.73 \mathrm{~mW}$ (green). The dashed line is the linear interpolated calibration curve at laser power $2.01 \mathrm{~mW}$ used for experiments described in Section 14. The calibration curve at $1.73 \mathrm{~mW}$ was used for experiments described in Section 15. Mean and standard deviation bars are calculated from the mean values of ROIs (Fig. S37). RFU distributions per pixel in the ROIs for (C) F302xdBroccoli RNA aptamer (LP 3.3) and (D) mCherry protein calibration standards. 

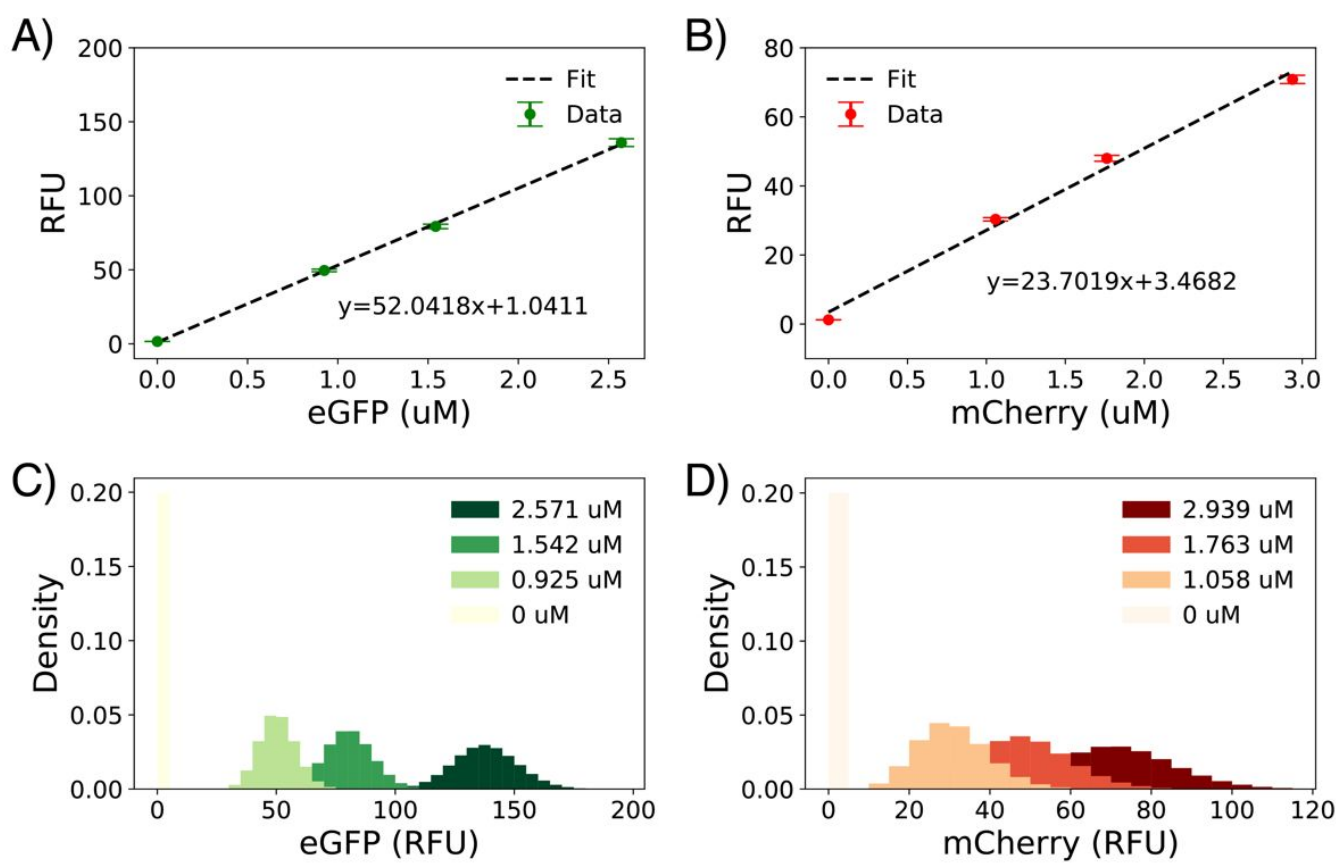

Figure S11. Calibration curve of (A) eGFP protein and (B) mCherry protein in PURExpress mastermix without DFHBI obtained from optical confocal images. Mean and standard deviations are calculated from the mean values of ROIs (Fig. S37). RFU distributions per pixel obtained from the ROIs for (C) eGFP protein and (D) mCherry protein calibration standards show differentiation between the fluorescence intensities of the standard proteins.

Table S17. Laser powers for quantified experiments.

\begin{tabular}{|c|c|c|c|}
\hline Experiment & Laser (nm) & Power (mW) & Section \\
\hline \multirow{3}{*}{$\begin{array}{l}\text { RNA Broccoli } \\
\text { calibration } 1\end{array}$} & 488 & 3.3 & \multirow{3}{*}{ S6 } \\
\hline & 561 & 3.96 & \\
\hline & 633 & 1.61 & \\
\hline \multirow{3}{*}{$\begin{array}{l}\text { eGFP and mCherry } \\
\text { protein calibration }\end{array}$} & 488 & 1.73 & \multirow{3}{*}{ S6 } \\
\hline & 561 & 3.22 & \\
\hline & 633 & 1.26 & \\
\hline \multirow{3}{*}{$\begin{array}{l}\text { RNA Broccoli } \\
\text { calibration } 2\end{array}$} & 488 & 1.73 & \multirow{3}{*}{ S6 } \\
\hline & 561 & 3.22 & \\
\hline & 633 & 1.26 & \\
\hline \multirow{3}{*}{$\begin{array}{l}\text { DNA titration in } \\
\text { synthetic cell populations }\end{array}$} & 488 & 2.01 & \multirow{3}{*}{ S14 } \\
\hline & 561 & 3.16 & \\
\hline & 633 & 1.12 & \\
\hline \multirow{3}{*}{$\begin{array}{l}\text { Two plasmids in a } \\
\text { synthetic population }\end{array}$} & 488 & 1.73 & \multirow{3}{*}{$\mathrm{S} 15$} \\
\hline & 561 & 3.22 & \\
\hline & 633 & 1.26 & \\
\hline
\end{tabular}




\section{Bulk cell-free expression experiments}

Bulk cell free expression experiments were undertaken using standard half-volume $(12.5 \mu \mathrm{L})$ reactions of the standard PURExpress In Vitro Protein Synthesis Kit. Reactions were prepared according to Table S18 for each sample, loaded into 384-well plates, sealed with an adhesive PCR plate clear film (Thermo, USA), and incubated in the TECAN Spark $20 \mathrm{M}$ plate reader at $30{ }^{\circ} \mathrm{C}$ for 8 hours. Fluorescence readings for the Broccoli RNA aptamer and mCherry protein, or eGFP protein and mCherry protein, were taken every 10 minutes with linear shaking of $5 \mathrm{~s}, 1 \mathrm{~mm}$ amplitude, and $1440 \mathrm{rpm}$ frequency at each cycle. Excitation/emission wavelengths were 485/535 nm (Gain 50), 570/620 nm (Gain 70), and 450/510 nm (Gain 60) with a bandwidth of $\pm 20 \mathrm{~nm}$ for eGFP, mCherry, and Broccoli RNA, respectively. DNA plasmid and mRNA templates were prepared by serial dilution with nuclease-free water before adding into the CFES reaction for the DNA (Fig. S12) and RNA titration experiments (Fig. S13). Transcription and translation rates from timeseries data are calculated using rate averages from a rolling window of 1 hour and plotted in Figs. S14-15.

Table S18. PURExpress master mix for $1 \mathrm{X}$ reaction

\begin{tabular}{cc}
\hline Component & Volume $(\boldsymbol{\mu l})$ \\
\hline Solution A & 5 \\
Solution B & 3.75 \\
RNAse Inhibitor & 0.25 \\
Sucrose $(1.5 \mathrm{M})$ & 0.67 \\
DFHBI $(500 \mu \mathrm{M})^{*}$ & 0.25 \\
Plasmid DNA or mRNA & $\mathrm{X}$ \\
Water & fill to 12.5 \\
\hline if using plasmids without F30-2xdBroccoli RNA aptamer.
\end{tabular}
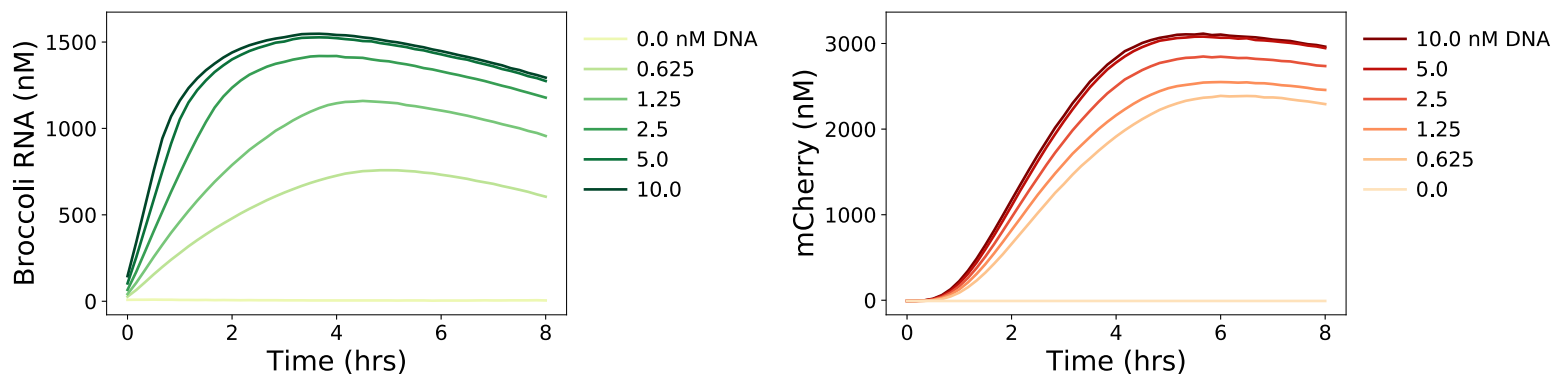

Figure S12. Timelapse of RNA Broccoli and mCherry protein expression in bulk PURExpress CFES at different pEXP5-NT/6xHis mCherry F30-2xdBroccoli plasmid DNA concentrations.
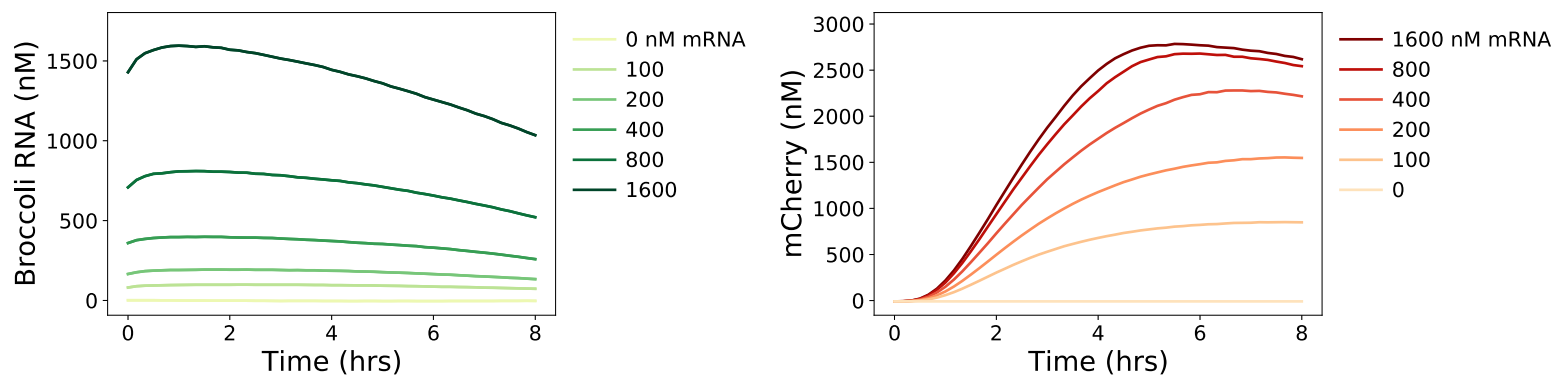

Figure S13. Timelapse of RNA Broccoli and mCherry protein expression in bulk PURExpress CFES at different 6xHis mCherry F30-2xdBroccoli transcript mRNA concentrations. 

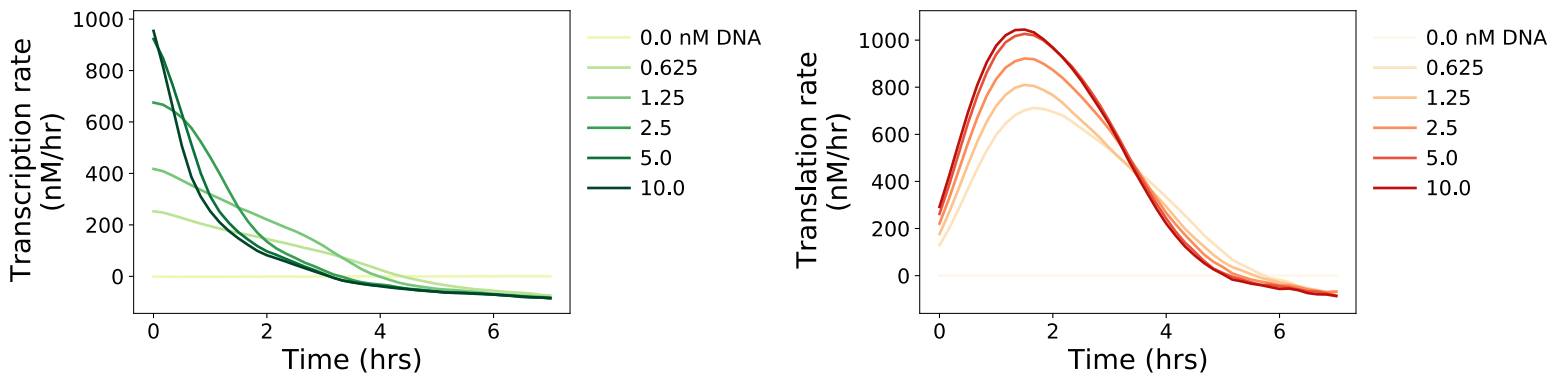

Figure S14. Transcription and translation rates from bulk PURExpress CFES at different pEXP5-NT/6xHis mCherry F30-2xdBroccoli plasmid DNA concentrations. Rates are calculated from results in Fig. S12 using rate averages with a rolling window of 1 hour.
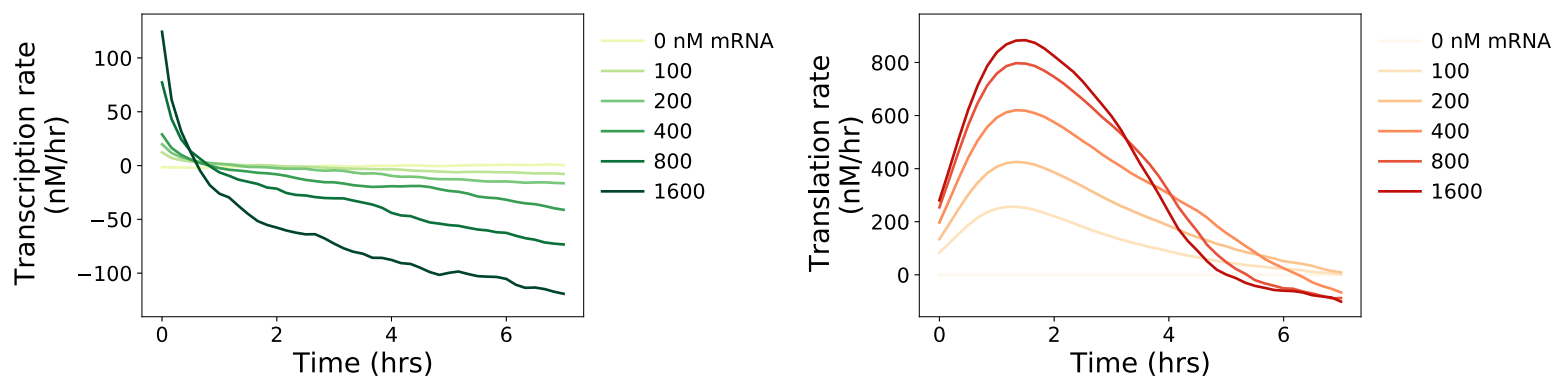

Figure S15. Transcription and translation rates from bulk PURExpress CFES at different 6xHis mCherry F302xdBroccoli transcript mRNA concentrations. Rates are calculated from results in Fig. S13 using rate averages with a rolling window of 1 hour. 


\section{7. mCherry maturation}

Maturation rate of the mCherry in PURExpress was measured using a modified RNAseA assay previously described in [6]. A PURExpress reaction was prepared with DFHBI (see Table S18) and $300 \mathrm{nM}$ 6xHis mCherry F30-2xdBroccoli mRNA template. The reaction was incubated at $30{ }^{\circ} \mathrm{C}$ for 30 mins to allow the CFES to produce mCherry protein. After 30 mins, $300 \mathrm{nM}$ of RNAseA was added into the CFES reaction to degrade all mRNA and halt translation. mRNA Broccoli aptamer and mCherry protein fluorescence values were monitored every 10 minutes in a TECAN Spark $20 \mathrm{M}$ plate reader while incubated at $30^{\circ} \mathrm{C}$ with linear shaking of $5 \mathrm{~s}, 1 \mathrm{~mm}$ amplitude, and $1440 \mathrm{rpm}$ frequency at each cycle (Fig. S16). Excitation/emission wavelengths used were 570/620 nm (Gain 70 ) and $450 / 510 \mathrm{~nm}$ (Gain 60) with a bandwidth of $\pm 20 \mathrm{~nm}$ each, for mCherry and Broccoli RNA, respectively. The maturation rate of mCherry was measured from the increase of mCherry signal after the RNA signal was depleted ( $\sim 2$ hours after the addition of RNAseA). The increase of mCherry signal is attributed to the maturation of the protein and fit to a first order kinetic model (Eqns. S1-2, Fig. S17). The mCherry maturation rate parameter obtained from the RNAseA assay is $k_{\text {mat }}=2.15 \pm 0.12 \mathrm{hr}^{-1}$.

A)

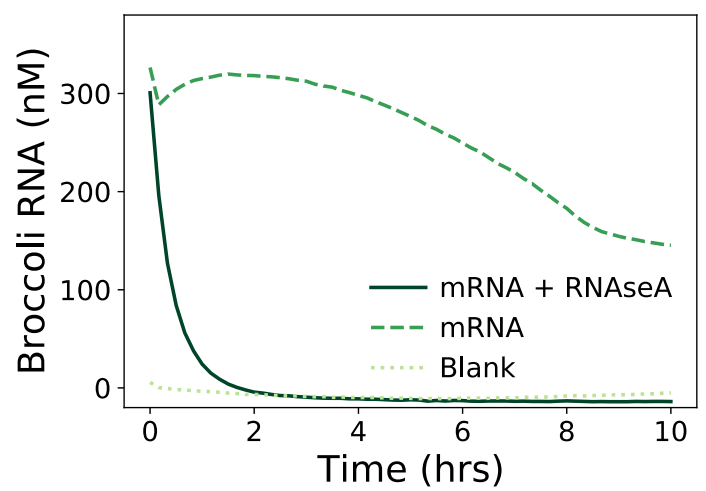

B)

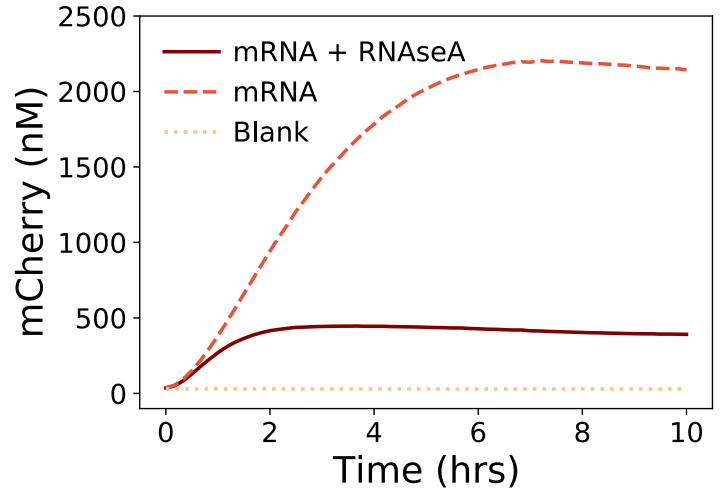

Figure S16. RNAseA assay for mCherry maturation rate. After 30 mins incubation of PURExpress with $6 x H i s$ mCherry F30-2xdBroccoli mRNA template, $300 \mathrm{nM}$ RNAseA was added to deplete mRNA and halt translation. Broccoli RNA aptamer (A) and mCherry protein (B) levels were monitored over time. Treatments without mRNA template and without RNAseA addition were included as controls. All samples were done in triplicate. Solid lines indicate mean values and shaded regions are within standard deviations. Time measured in the $\mathrm{x}$-axis is upon addition of RNAseA.

$$
\begin{gathered}
\frac{d\left[\text { Protein }^{*}\right]}{d t}=k_{\text {mat }}[\text { Protein }] \\
{\left[\text { Protein }^{*}\right]=\left[\text { Protein }^{*}\right]_{0}+[\text { Protein }]_{0}\left(1-e^{-k_{\text {mat }} t}\right)}
\end{gathered}
$$

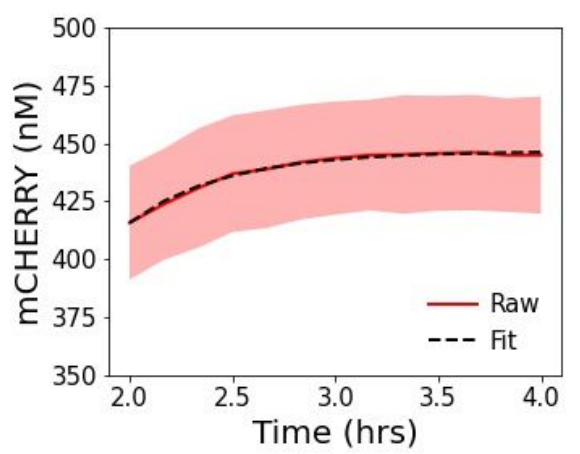

Figure S17. Fit of the mCherry signal to a first order kinetic model (Eqn. S2) for protein maturation from 2-4 hours after addition of RNAseA. Fit parameters are $k_{\text {mat }}=2.15 \pm 0.12 \mathrm{hr}^{-1}$ and [Protein $]_{0}=30.72 \pm 0.47 \mathrm{nM}$ 


\section{Resource-limited gene expression models}

Seven different candidate models for cell-free gene expression (summarized in Table S19), based on and including the resource-limited gene expression model in [7], were used to fit our bulk and encapsulated RNA Broccoli and mCherry protein expression timeseries data. The general structure of the models consists of either mass-action, Michaelis-Menten, or Hill kinetics to describe the different steps of transcription and translation to provide a coarse-grained model of gene expression. In addition, gene expression was modeled as a resource-limited process. Transcription and translation processes consume $T s R$ and $T l R$ species, which are transcription and translation resources, respectively. To illustrate, the resource-limited gene expression model can be described by the reaction network with their respective reaction rate constants in Eqns. S3-10.

$$
\begin{gathered}
D N A+R N A P \stackrel{k_{f 1} / k_{b 1}}{\longrightarrow} D N A: R N A P \\
D N A: R N A P+T s R \stackrel{k_{c a t 1}}{\longrightarrow} D N A+R N A P+R N A \\
R N A+R b \stackrel{k_{f 2} / k_{b 2}}{\longrightarrow} R N A: R b \\
R N A: R b+T l R \stackrel{k_{c a t 2}}{\longrightarrow} R N A+R b+\text { Protein } \\
\text { Protein } \stackrel{k_{\text {mat }}}{\longrightarrow} \text { Protein } * \\
R N A \stackrel{\delta_{R N A}}{\longrightarrow} \\
T s R \stackrel{\delta_{T S R}}{\longrightarrow} \\
T l R \stackrel{\delta_{T l R}}{\longrightarrow}
\end{gathered}
$$

For transcription, $D N A$ is first reversibly bound to $R N A$ polymerase (RNAP) to form a $D N A: R N A P$ complex (Eqn. $\mathrm{S} 3$ ). This complex can consume transcription resources, TsR, to produce $R N A$ (Eqn. S4). In the process, $D N A$ and $R N A P$ dissociate from each other. Following mass-action kinetics, the rate equation for $R N A$ production can be written as

$$
\frac{d[R N A]}{d t}=k_{c a t 1}[D N A: R N A P][T s R]
$$

Assuming that the binding/unbinding of $D N A$ and $R N A P$ is fast and in quasi-equilibrium

$$
k_{f 1}[D N A][R N A P]=k_{b 1}[D N A: R N A P] .
$$

Additionally, assuming that total RNAP concentration is conserved,

$$
\begin{gathered}
k_{f 1}[D N A]\left([R N A P]_{0}-[D N A: R N A P]\right)=k_{b 1}[D N A: R N A P] \\
{[D N A: R N A P]=\frac{k_{f 1}[D N A][R N A P]_{0}}{k_{b 1}+k_{f 1}[D N A]}}
\end{gathered}
$$

where $[R N A P]_{0}$ is the initial amount of $R N A$ polymerase in the reaction. Replacing $[D N A: R N A P]$ in the rate equation of $R N A$ production and grouping constants together results in

$$
\begin{gathered}
\frac{d[R N A]}{d t}=\frac{k_{c a t 1} k_{f 1}[D N A][R N A P]_{0}[T s R]}{k_{b 1}+k_{f 1}[D N A]} \\
\frac{d[R N A]}{d t}=\frac{k_{r}[D N A][T s R]}{K_{r}+[D N A]}
\end{gathered}
$$

where $k_{r}=k_{\text {cat } 1}[R N A P]_{0}$ is an effective rate constant for transcription and $K_{r}=k_{b 1} / k_{f 1}$ is the dissociation constant between $D N A$ and $R N A P$. A similar derivation can be performed for translation of protein (Eqns. S5-6) to obtain

$$
\frac{d[\text { Protein }]}{d t}=\frac{k_{p}[\text { Protein }][\text { TlR }]}{K_{p}+[\text { Protein }]}
$$

where $k_{p}$ is an effective rate constant for translation and $K_{p}$ is the dissociation constant between $R N A$ and ribosome, $R b$. The remaining steps for protein maturation, $R N A$ degradation (Eqn. S8), TsR degradation (Eqn. 
S9), and TlR degradation (Eqn. S10) in the reaction network are modeled as first order reactions. Altogether, the reaction network of Eqns. S3-10 can be written as a system of ordinary differential equations (Eqns. S18-23)

$$
\begin{gathered}
\frac{d[D N A]}{d t}=0 \\
\frac{d[R N A]}{d t}=\frac{k_{r} T s R[D N A]}{K_{r}+[D N A]}-\delta_{r}[R N A] \\
\frac{d[\text { Protein }]}{d t}=\frac{k_{p} T l R[R N A]}{K_{p}+[R N A]}-k_{\text {mat }}[\text { Protein }] \\
\frac{d\left[\text { Protein }{ }^{*}\right]}{d t}=k_{\text {mat }}[\text { Protein }] \\
\frac{d T s R}{d t}=-\frac{a k_{r} T s R[D N A]}{K_{r}+[D N A]}-\delta_{T s R} T s R \\
\frac{d T l R}{d t}=-\frac{b k_{p} T l R[R N A]}{K_{p}+[R N A]}-\delta_{T l R} T l R
\end{gathered}
$$

where $T s R$ and $T l R$ are assigned unitless quantities starting from 1 and are consumed during transcription and translation, scaled by the factors $a$ and $b$, respectively. Delay differential equations can be used to describe translation to account for the $\sim 0.5$ hour delay of protein expression observed in mRNA titration bulk experiments. For example, Eqn. 20 can be rewritten to include a time-delay, $\tau_{d}$, as

$$
\frac{d[\text { Protein }]}{d t}=\frac{k_{p} T l R[R N A]\left(t-\tau_{d}\right)}{K_{p}+[R N A]\left(t-\tau_{d}\right)}-k_{\text {mat }}[\text { Protein }]
$$

where $[R N A]\left(t-\tau_{d}\right)$ is the $R N A$ abundance at time $\left(t-\tau_{d}\right)$.

Different combinations of mass-action or Michaelis-Menten kinetics are used for the degradation of $T s R$ and $T l R$ in the candidate models. However, unlike the Michaelis-Menten kinetics of transcription and translation, we do not derive $T S R$ and $T l R$ degradation kinetics from a particular reaction network because $T s R$ and $T l R$ are abstracted species of transcription and translation resources. We chose to represent their degradation dynamics as either first order or Michaelis-Menten kinetics for simplicity. For example, model 1 is shown in Eqns. S25-30, which includes Michaelis-Menten type kinetics for resource degradation (2nd terms on Eqns. S29 and S30).

Model 7 is the model provided by Stögbauer et al. (2012)[7] without a delay differential equation for translation, while model 5 includes a delay differential equation for the translation step. Each parameter is described in Table S20.

$$
\begin{aligned}
& \frac{d[D N A]}{d t}=0 \\
& \frac{d[R N A]}{d t}=\frac{k_{r} T S R[D N A]}{K_{r}+[D N A]}-\delta_{r}[R N A] \\
& \frac{d[\text { Protein }]}{d t}=\frac{k_{p} T l R[R N A]\left(t-\tau_{d}\right)}{K_{p}+[R N A]\left(t-\tau_{d}\right)}-k_{\text {mat }}[\text { Protein }] \\
& \frac{d\left[\text { Protein }^{*}\right]}{d t}=k_{\text {mat }}[\text { Protein }] \\
& \frac{d T s R}{d t}=-\frac{a k_{r} T s R[D N A]}{K_{r}+[D N A]}-\frac{\delta_{T s R} T s R}{K_{s}+T s R} \\
& \frac{d T l R}{d t}=-\frac{b k_{p} T l R[R N A]\left(t-\tau_{d}\right)}{K_{p}+[R N A]\left(t-\tau_{d}\right)}-\frac{\delta_{T l R} T l R}{K_{l}+T l R}
\end{aligned}
$$


Table S19. Summary of seven candidate CFES models. Each model is composed of the different reaction steps modeled by mass-action kinetics (MA), Michaelis-Menten kinetics (MM), or Michaelis-Menten kinetics with delay (DM). Model 7 is the model provided in Stögbauer et al. (2012) [7].

\begin{tabular}{|c|c|c|c|c|c|c|c|}
\hline Step & $\begin{array}{c}\text { Model } \\
1\end{array}$ & $\begin{array}{c}\text { Model } \\
2\end{array}$ & $\begin{array}{c}\text { Model } \\
3\end{array}$ & $\begin{array}{c}\text { Model } \\
4\end{array}$ & $\begin{array}{c}\text { Model } \\
5\end{array}$ & $\begin{array}{c}\text { Model } \\
6\end{array}$ & $\begin{array}{c}\text { Model } \\
7\end{array}$ \\
\hline Transcription & MM & MM & MM & MM & MM & MM & MM \\
\hline Translation & $\mathrm{DM}$ & $\mathrm{DM}$ & $\mathrm{DM}$ & $\mathrm{DM}$ & $\mathrm{DM}$ & $\mathrm{DM}$ & MM \\
\hline RNA degradation & MA & MA & MA & MA & MA & MA & MA \\
\hline Protein maturation & MA & MA & MA & MA & MA & MA & MA \\
\hline TsR consumption & MM & MM & MM & MM & MM & MM & MM \\
\hline T1R consumption & DM & DM & $\mathrm{DM}$ & - & - & $\mathrm{DM}$ & - \\
\hline TsR degradation & MM & MA & MM & MM & - & - & - \\
\hline TIR degradation & MM & MM & MA & - & MM & - & MM \\
\hline
\end{tabular}

Table S20. Rate parameters of resource-limited gene expression models.

\begin{tabular}{ccl}
\hline Parameter & Units & Description \\
\hline$k_{r}$ & $\mathrm{nM} / \mathrm{hr}$ & RNA transcription rate. \\
$K_{r}$ & $\mathrm{nM}$ & Dissociation constant between $R N A P$ and $D N A$. \\
$\delta_{r}$ & $1 / \mathrm{hr}$ & Degradation rate of $R N A$. \\
$k_{p}$ & $\mathrm{nM} / \mathrm{hr}$ & Protein translation rate. \\
$K_{p}$ & $\mathrm{nM}$ & Dissociation constant between ribosome and $R N A$. \\
$k_{m a t}$ & $1 / \mathrm{hr}$ & Protein maturation rate. \\
$\delta_{T s R}$ & $1 / \mathrm{hr}$ & $T s R$ degradation rate. \\
$K_{s}$ & - & Michaelis-Menten constant of $T s R$ degradation. \\
$\delta_{T l R}$ & $1 / \mathrm{hr}$ & TlR degradation rate. \\
$K_{l}$ & - & Michaelis-Menten constant of $T l R$ degradation. \\
$a$ & - & Scaling factor for consumption of $T s R$ with \\
& & transcription. \\
$b$ & - & Scaling factor for consumption of $T l R$ with \\
$\tau_{d}$ & $\mathrm{hr}$ & translation. \\
\hline
\end{tabular}




\section{Parameter estimation, model selection, and profile likelihoods}

All methods for parameter estimation, model selection, and profile likelihood analysis were written in Julia (v1.5.3) [8] using the packages DifferentialEquations, Distributions, Optim, Plots, DataFrames, DelimitedFiles, and CSV.

\section{Parameter estimation}

We obtained parameter estimates of each model by maximizing the agreement between the experimental data and model. This agreement can be quantified by the likelihood $p(D \mid \theta)$, defined as the probability of observing the experimental data $D$ given the model parameters $\theta=\left\{\theta_{1}, \cdots, \theta_{k}\right\}$. The specific form of the likelihood $p(D \mid \theta)$ results from the considered measurement noise model. Throughout this work, we consider both Broccoli RNA and mCherry measurements to be corrupted by independent Gaussian measurement noise with standard deviation $\sigma_{r}$ and $\sigma_{p}$, respectively. The rate parameters of a model were estimated by minimizing the negative logarithm of the likelihood

$$
\hat{\theta}=\operatorname{argmin}_{\theta}[-\ln p(D \mid \theta)]
$$

where $\hat{\theta}$ is referred to as the maximum-likelihood estimator of the model parameters $\theta$. All rate parameters in each model were estimated simultaneously in the log domain to avoid negative parameter values. In addition to the rate parameters, the unknown standard deviations for Broccoli RNA and mCherry protein mean fluorescence signal ( $\sigma_{r}$ and $\left.\sigma_{p}\right)$ and the time lag between reaction start and data collection $\left(\tau_{d}\right)$ were also estimated from the data. The rate parameter for mCherry maturation $\left(k_{\text {mat }}\right)$ was determined from independent experiments described in Section 8. The negative logarithm of the likelihood was minimized using a Nelder-Mead algorithm where ODE or DDE models were solved using a Bogacki-Shampine Runge-Kutta method. Initial parameters used are reported in the supplementary files and were taken from pre-search runs to facilitate better convergence of the optimization procedure.

AIC model selection

To rank the seven candidate models, we calculated the Akaike information criterion (AIC) defined as

$$
\mathrm{AIC}=2 k-2 \ln p(D \mid \hat{\theta})
$$

where $k$ is the number of estimated parameters and $\ln p(D \mid \hat{\theta})$ is the log-likelihood evaluated at the optimal set of parameters $\hat{\theta}$. The AIC values of each model are shown in Table S21. Fits of the models with bulk experiments are shown in Figs. S18-24. Model 7 is the same model provided by Stögbauer et al. (2012) [7], but resulted in the highest AIC (and lowest log-likelihood). This was due to the initial delay of mCherry signal in both DNA and RNA titration experiments and endpoints of mRNA in the DNA titration experiments not fitting well. We then modified this model to include a delay in translation (models 1-6) and degradation of transcription resources (models 1-4) to account for the observed deviations from experimental data. These model modifications improved the AIC (Table S21) and qualitative fit (Figs. S18-24). Overall, model 2 was selected to be presented in the main text because it had the lowest AIC score, which accounts for both goodness-of-fit through the log-likelihood score, as well as model complexity by a penalty term that increases with the number of unknown model parameters.

Profile likelihoods and confidence intervals

Profile likelihoods for models were calculated according to $[9,10]$ to determine identifiability and confidence intervals of each parameter. The profile likelihood of a parameter is calculated by

$$
\operatorname{PL}\left(\theta_{i}\right)=\min _{\theta_{j \neq i}}[-\ln p(D \mid \theta)]
$$

which is the minimum of the negative log-likelihood with respect to all parameters $\theta_{j \neq i}$ while holding the parameter $\theta_{i}$ fixed [9]. Likelihood-based confidence intervals were estimated as the regions with

$$
\left\{\theta_{i} \mid \operatorname{PL}\left(\theta_{i}\right)+\ln p(D \mid \hat{\theta})<\chi^{2}(\alpha, d f)\right\}
$$

where $\chi^{2}(\alpha, d f)$ is the chi-squared distribution with $\alpha=0.95$ confidence level and $d f$ degrees of freedom, which here is the number of parameters. Graphically, this means that the confidence intervals are found at the intersections of the profile likelihood and the significance threshold horizontal line at $-\ln p(D \mid \hat{\theta})+\chi^{2}(\alpha, d f)$. Profile likelihoods of each parameter between a range greater and less than $\hat{\theta}$ by a factor of 10 at 11 equally spaced increments in of models 1-7 are shown in Figs. S25-30. The 95\% likelihood-based confidence intervals of the 
parameters from the profile likelihoods (Table 1 in the main text) are calculated from the upper and lower intersection of the profile likelihood and the significance threshold line. Structural non-identifiable parameters are indicated by flat profile likelihoods below the significance threshold in both $-\infty /+\infty$ directions. Practically nonidentifiable parameters are indicated by profile likelihoods below the significance threshold in both or either $\infty /+\infty$ directions, but still have a unique minimum [9].

Table S21. Log-likelihood and AIC of the different CFES models based on data from bulk experiments.

\begin{tabular}{|c|c|}
\hline Model & Log-likelihood \\
\hline 1 & -6822.78 \\
\hline 2 & -6822.87 \\
\hline 3 & -6887.60 \\
\hline 4 & -6922.83 \\
\hline 5 & -7255.11 \\
\hline 6 & -7208.64 \\
\hline 7 & -7354.29 \\
\hline \multirow[t]{2}{*}{ 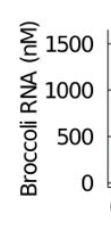 } & \\
\hline & ${ }^{2} \underset{\text { Time (hrs) }}{4} 68$ \\
\hline
\end{tabular}

\begin{tabular}{cc} 
Parameters & AIC \\
\hline 15 & 13675.56 \\
14 & 13673.74 \\
14 & 13803.21 \\
14 & 13873.65 \\
12 & 14534.21 \\
\hline 11 & 14439.28 \\
\hline 11 & 14730.58 \\
\hline
\end{tabular}
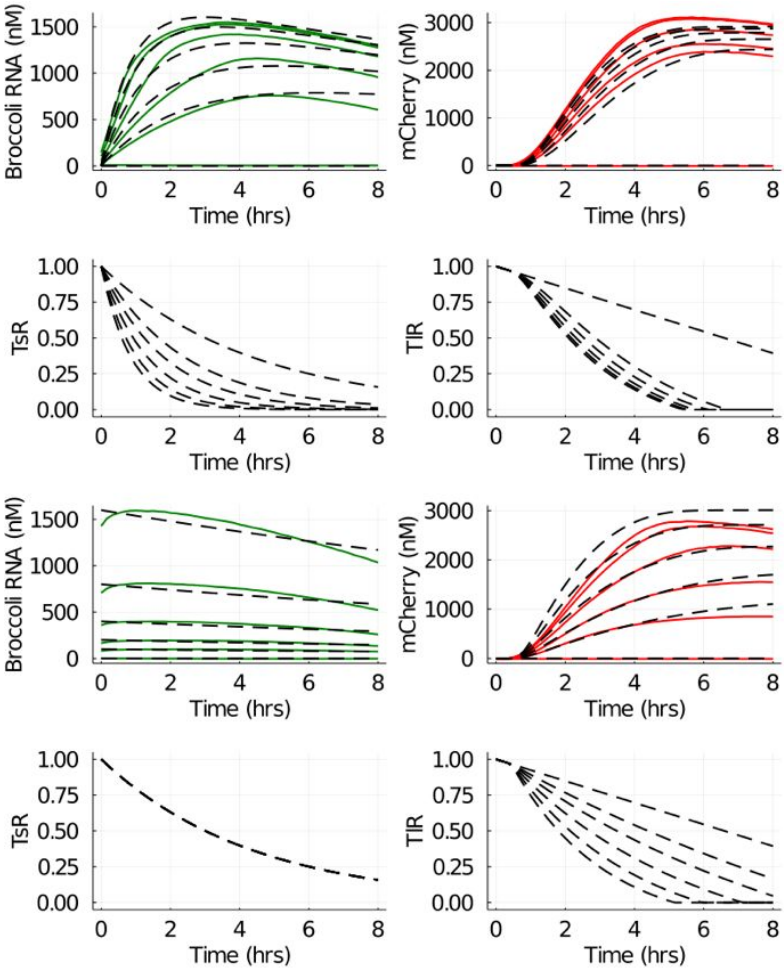

Figure S18. Model 1 fit with DNA titration and RNA titration bulk experiments. Colored lines are mean timeseries experimental data for the different species $R N A$, Protein, $T s R$, and $T l R$. Dashed lines are the model simulation from the parameter estimates. 

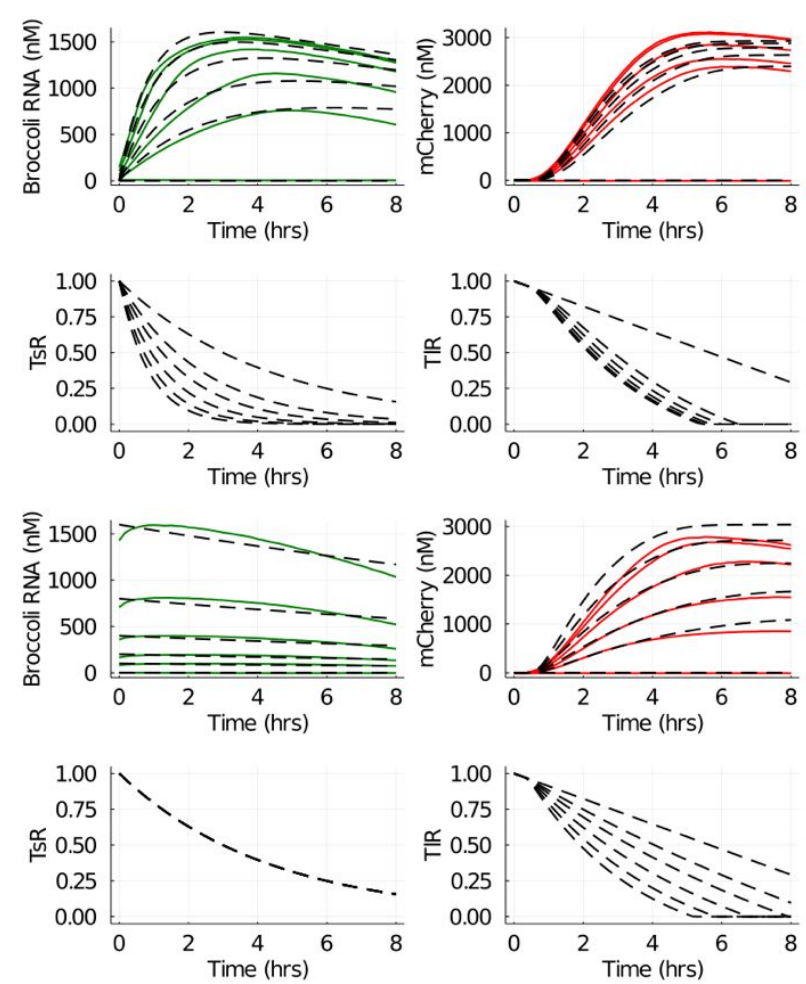

Figure S19. Model 2 fit with DNA titration and RNA titration bulk experiments. Colored lines are mean timeseries experimental data for the different species $R N A$, Protein, $T s R$, and $T l R$. Dashed lines are the model simulation from the parameter estimates.
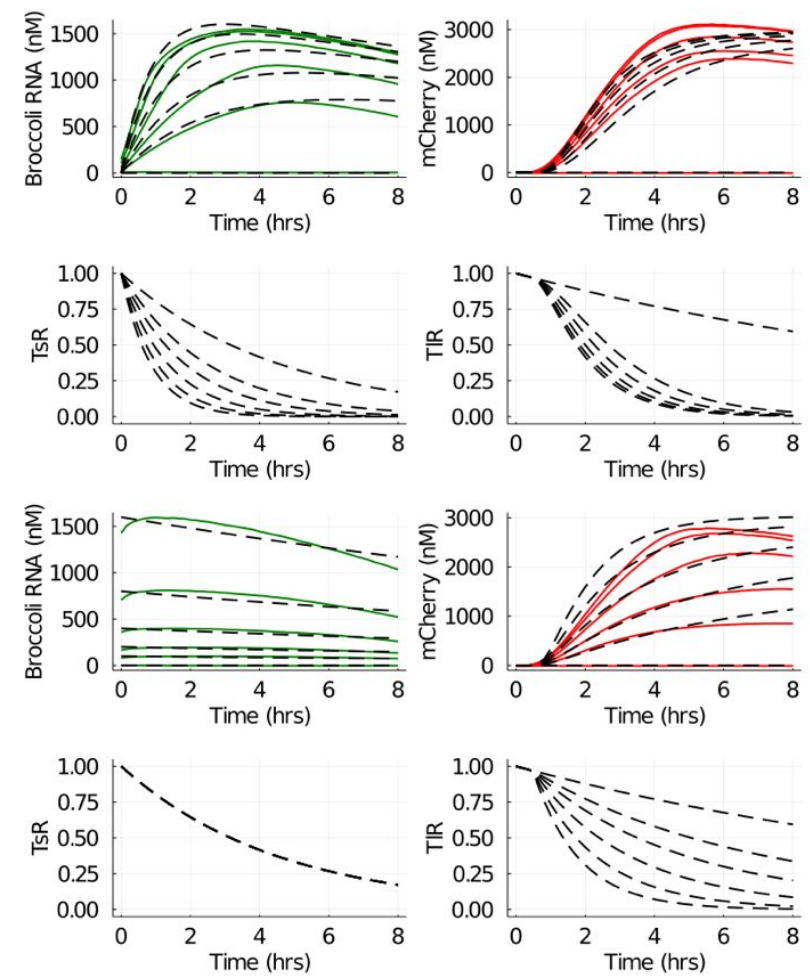

Figure S20. Model 3 fit with DNA titration and RNA titration bulk experiments. Colored lines are mean timeseries experimental data for the different species $R N A$, Protein, $T s R$, and $T l R$. Dashed lines are the model simulation from the parameter estimates. 

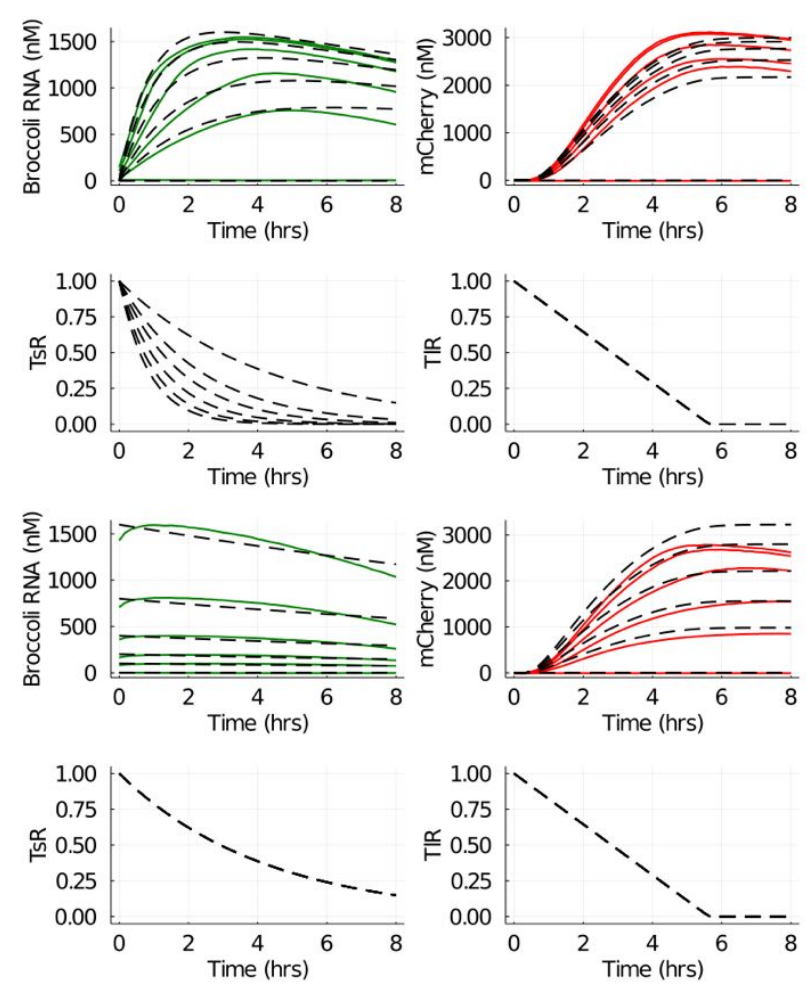

Figure S21. Model 4 fit with DNA titration and RNA titration bulk experiments. Colored lines are mean timeseries experimental data for the different species $R N A$, Protein, $T s R$, and $T l R$. Dashed lines are the model simulation from the parameter estimates.
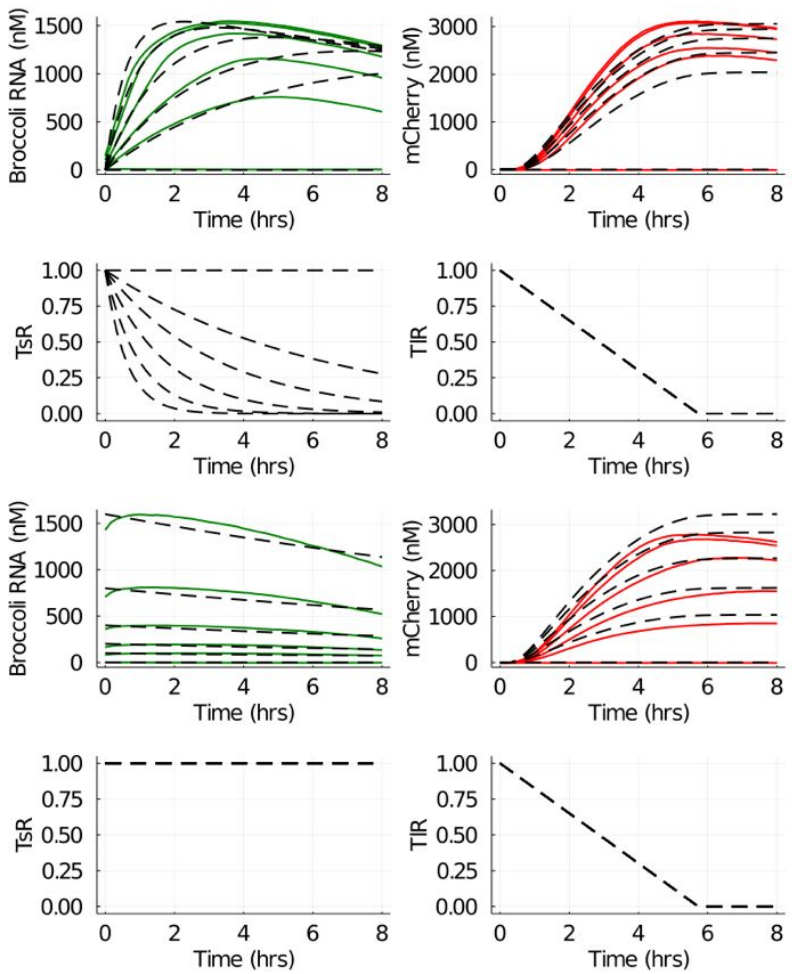

Figure S22. Model 5 fit with DNA titration and RNA titration bulk experiments. Colored lines are mean timeseries experimental data for the different species $R N A$, Protein, $T s R$, and $T l R$. Dashed lines are the model simulation from the parameter estimates. 

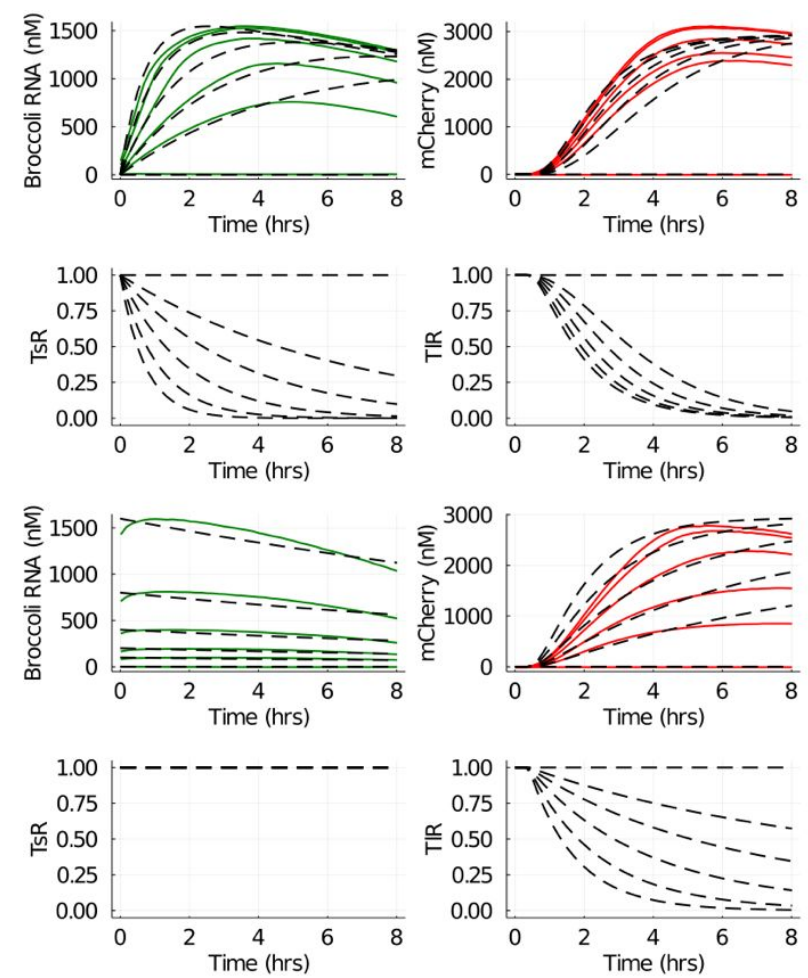

Figure S23. Model 6 fit with DNA titration and RNA titration bulk experiments. Colored lines are mean timeseries experimental data for the different species $R N A$, Protein, $T s R$, and $T l R$. Dashed lines are the model simulation from the parameter estimates.
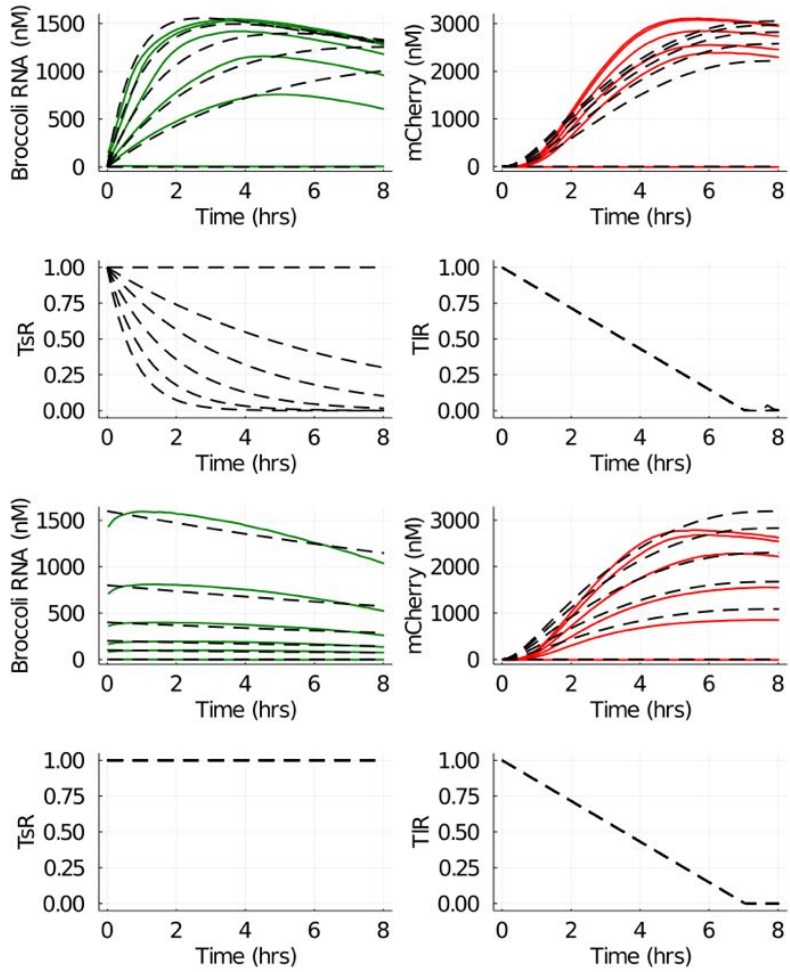

Figure S24. Model 7 fit with DNA titration and RNA titration bulk experiments. Colored lines are mean timeseries experimental data for the different species $R N A$, Protein, $T s R$, and $T l R$. Dashed lines are the model simulation from the parameter estimates. 

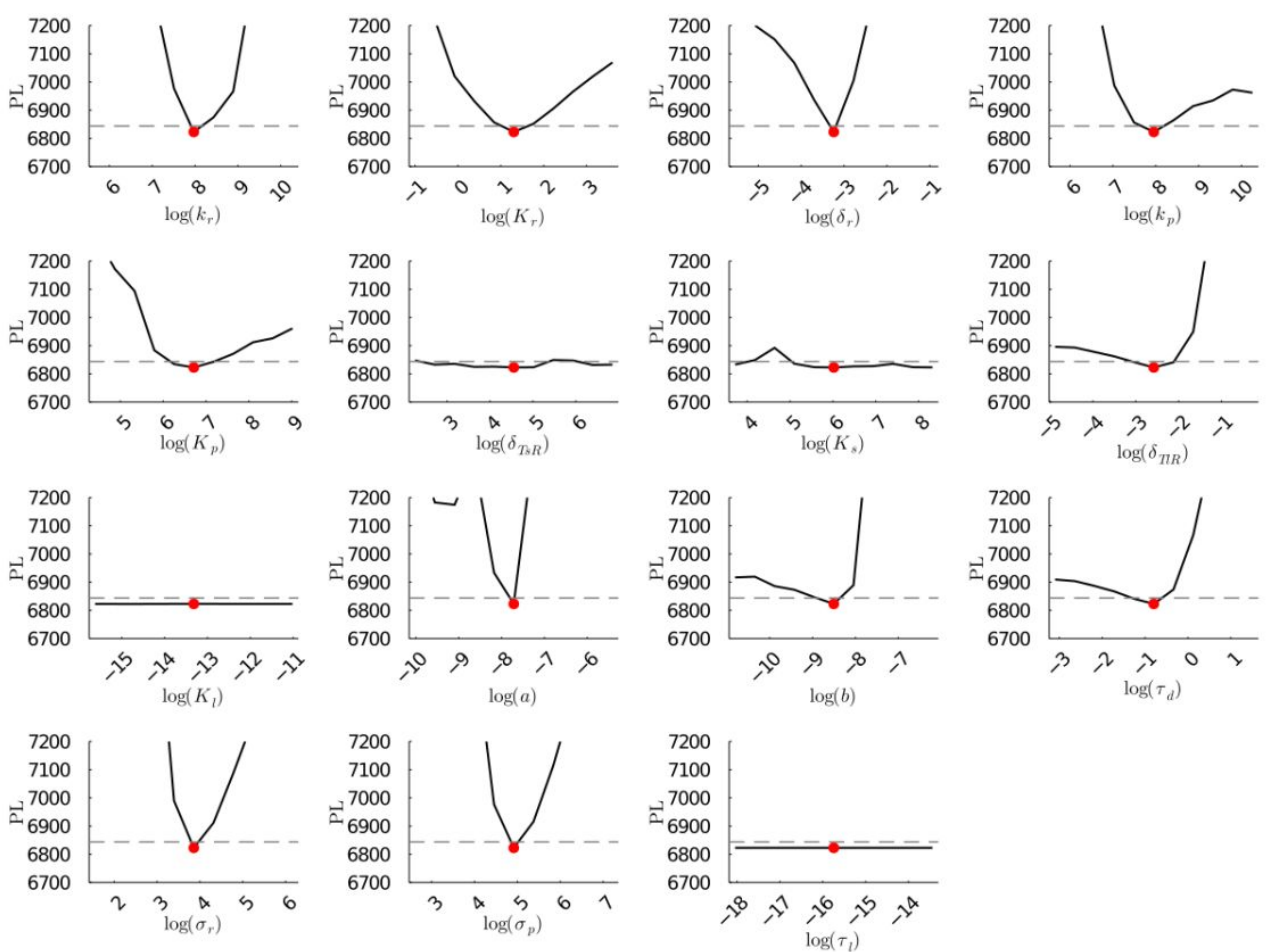

Figure S25. Profile likelihoods of parameters from model 1. Each plot corresponds to a parameter in the model and additional fitting parameters $\left(\sigma_{r}, \sigma_{p}\right.$, and $\left.\tau_{d}\right)$. The y-axis of each plot shows the minimized negative loglikelihood of the model with respect to all parameters except for the parameter given its value in the $\mathrm{x}$-axis. The red dot shows the optimized parameter set with the negative MLE. The dashed grey line is the $95 \%$ significance threshold line. All logarithms are in natural log.
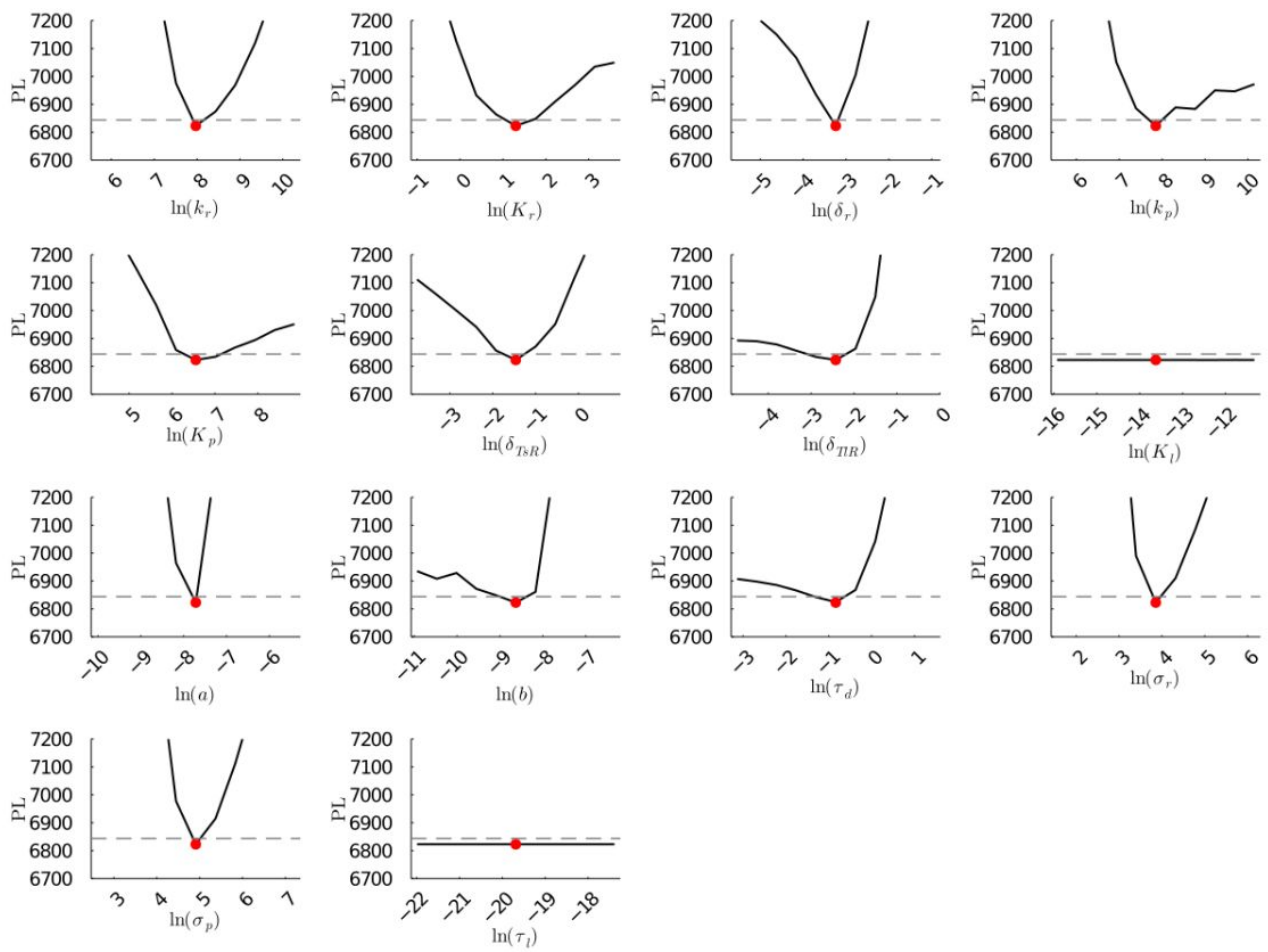

Figure S26. Profile likelihoods of parameters from model 2. Each plot corresponds to a parameter in the model and additional fitting parameters $\left(\sigma_{r}, \sigma_{p}\right.$, and $\left.\tau_{d}\right)$. The y-axis of each plot shows the minimized negative loglikelihood of the model with respect to all parameters except for the parameter given its value in the $\mathrm{x}$-axis. The red dot shows the optimized parameter set with the negative MLE. The dashed grey line is the $95 \%$ significance threshold line. All logarithms are in natural log. 

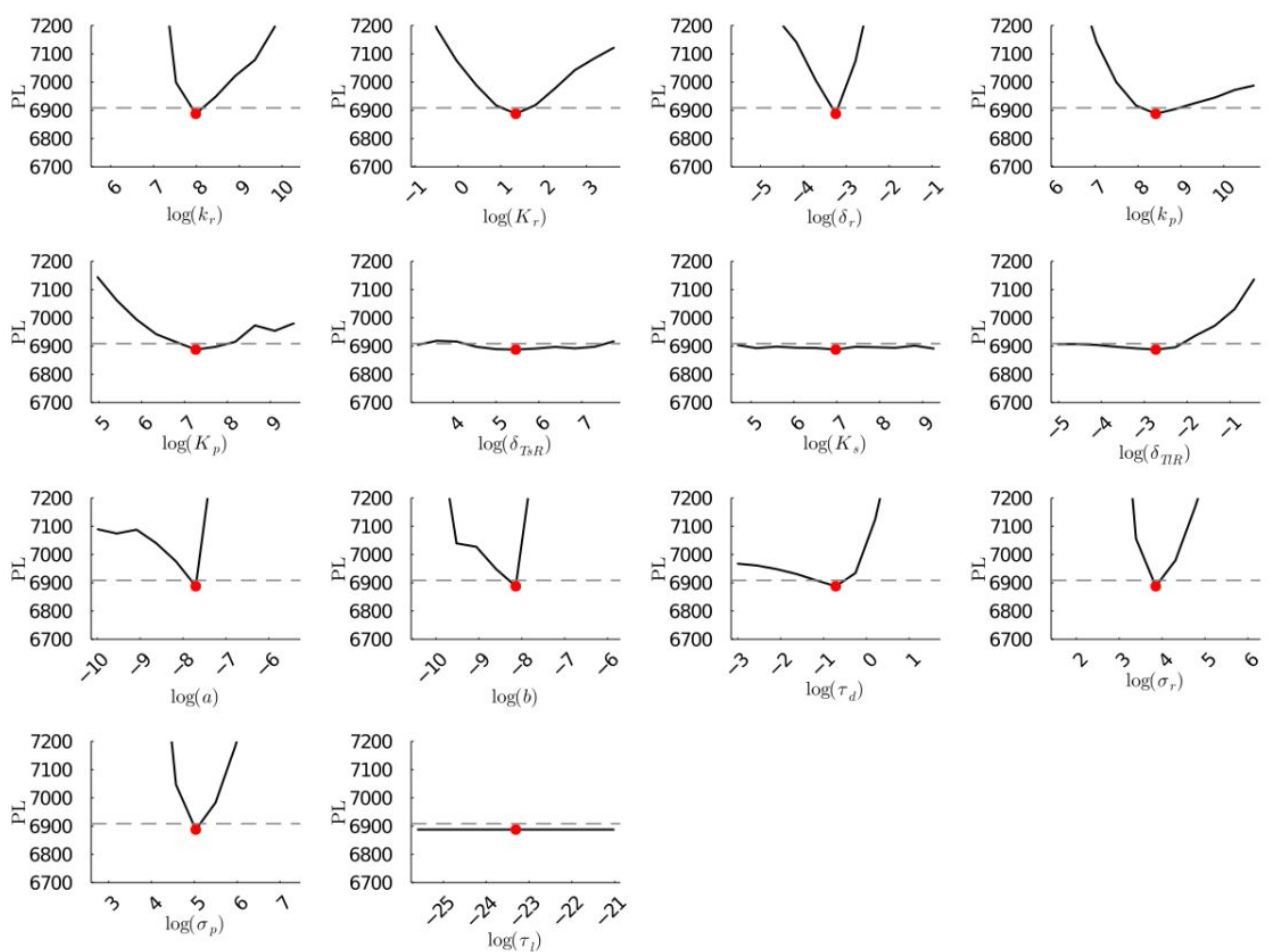

Figure S27. Profile likelihoods of parameters from model 3. Each plot corresponds to a parameter in the model and additional fitting parameters $\left(\sigma_{r}\right.$ and $\left.\sigma_{p}\right)$. The y-axis of each plot shows the minimized negative log-likelihood of the model with respect to all parameters except for the parameter given its value in the $\mathrm{x}$-axis. The red dot shows the optimized parameter set with the negative MLE. The dashed grey line is the $95 \%$ significance threshold line. All logarithms are in natural log.
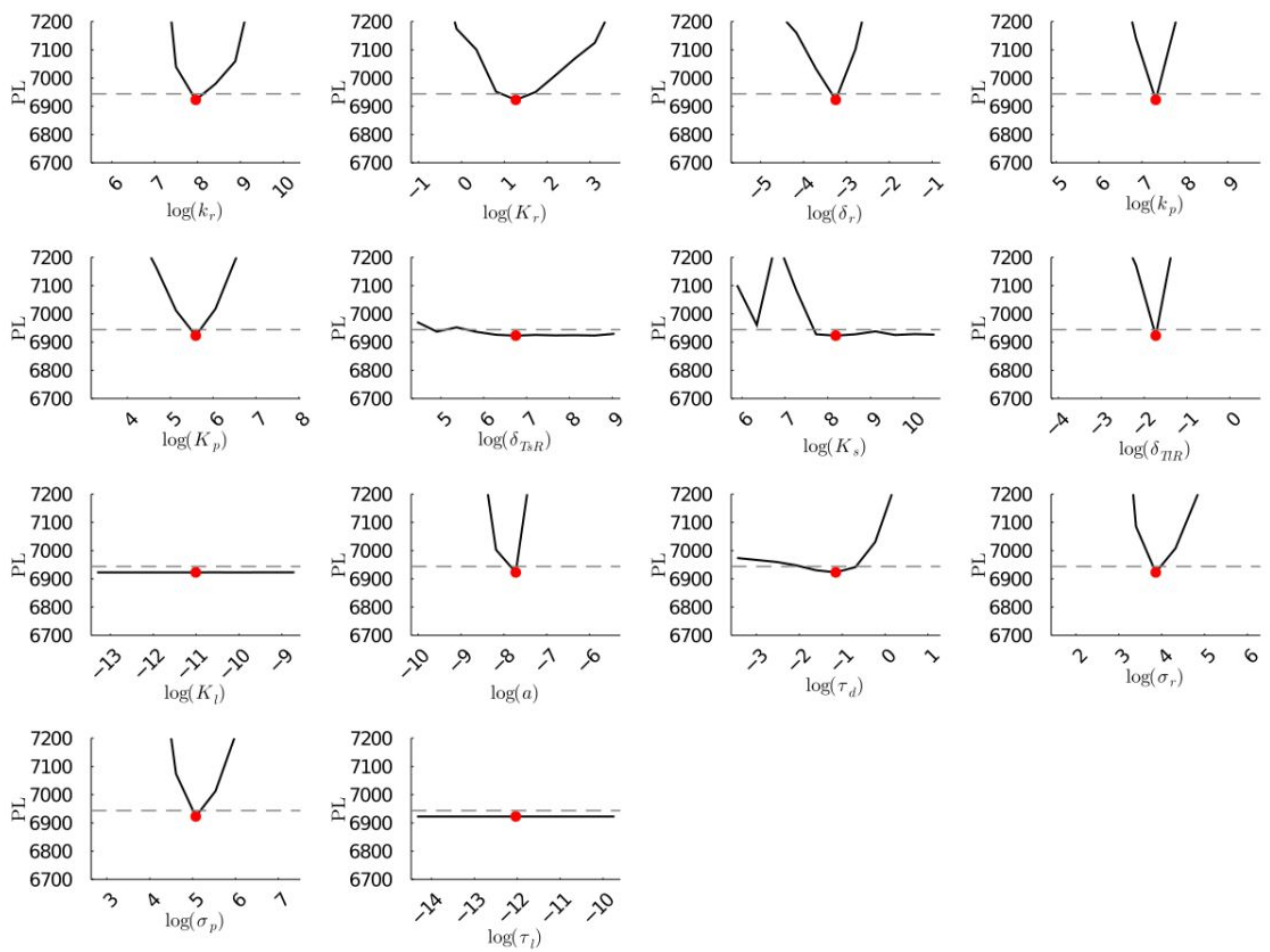

Figure S28. Profile likelihoods of parameters from model 4. Each plot corresponds to a parameter in the model and additional fitting parameters $\left(\sigma_{r}\right.$ and $\left.\sigma_{p}\right)$. The y-axis of each plot shows the minimized negative log-likelihood of the model with respect to all parameters except for the parameter given its value in the $\mathrm{x}$-axis. The red dot shows the optimized parameter set with the negative MLE. The dashed grey line is the $95 \%$ significance threshold line. All logarithms are in natural log. 

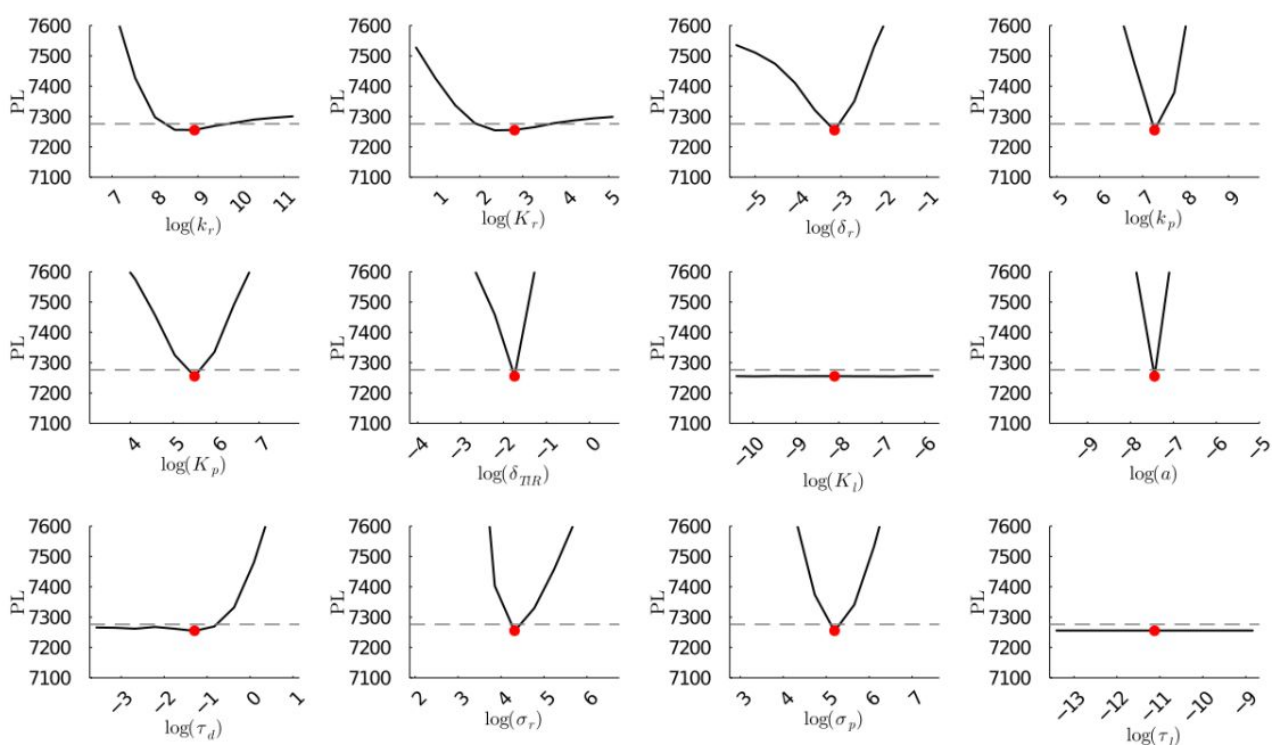

Figure S29. Profile likelihoods of parameters from model 5. Each plot corresponds to a parameter in the model and additional fitting parameters $\left(\sigma_{r}\right.$ and $\left.\sigma_{p}\right)$. The y-axis of each plot shows the minimized negative log-likelihood of the model with respect to all parameters except for the parameter given its value in the $\mathrm{x}$-axis. The red dot shows the optimized parameter set with the negative MLE. The dashed grey line is the $95 \%$ significance threshold line. All logarithms are in natural log.
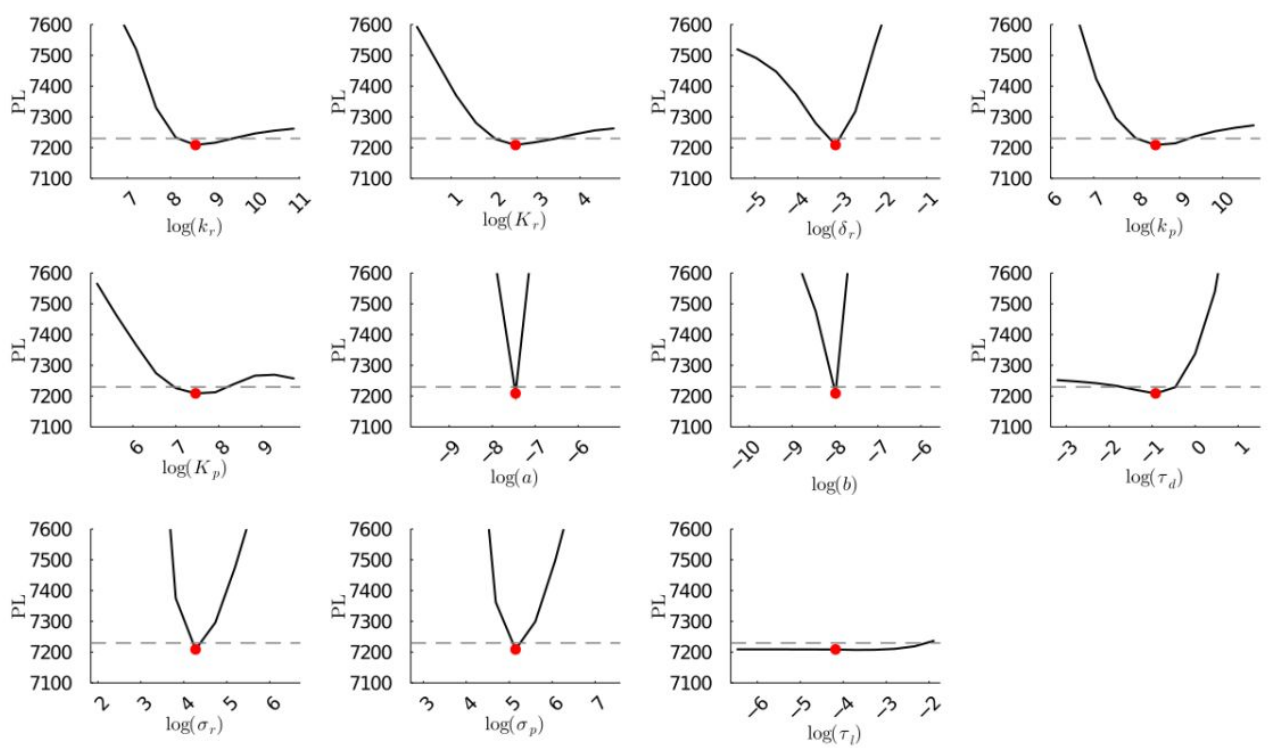

Figure S30. Profile likelihoods of parameters from model 6. Each plot corresponds to a parameter in the model and additional fitting parameters $\left(\sigma_{r}\right.$ and $\left.\sigma_{p}\right)$. The y-axis of each plot shows the minimized negative log-likelihood of the model with respect to all parameters except for the parameter given its value in the $\mathrm{x}$-axis. The red dot shows the optimized parameter set with the negative MLE. The dashed grey line is the $95 \%$ significance threshold line. All logarithms are in natural log. 


\section{Microfluidic chip fabrication and pretreatment}

PDMS microfluidic chips were designed and fabricated as presented in [11] using soft-photolithography methods. Briefly, 4" Si-wafer master molds were prepared by spin coating (model no. WS-650MZ- 23NPPB, Laurell Tech. Corp.) SU8 2025 (Microchem Inc.) to a height of $50 \mu \mathrm{m}$. The SU8-coated Si-wafers were pre-baked, exposed with UV-light through a film mask with the requisite design (see supplementary CAD file in [11]) for $7 \mathrm{~s}$ (MicroLithography Services), and then post-baked. SU8 development was then done by gently washing the wafer in developer solution (Microchem Inc.) for 3 mins before hard-baking the Si-wafers for 30 min at $200{ }^{\circ} \mathrm{C}$. Lastly, the prepared master molds were silanized overnight $(50 \mu \mathrm{l}$ of $1 \mathrm{H}, 1 \mathrm{H}, 2 \mathrm{H}, 2 \mathrm{H}$-perfluorodecyltrichlorosilane) in a desiccator. PDMS with curing agent (10:1 mix) was degassed for 30 mins and poured over the prepared master molds. The PDMS was cured for 3 hours at $90{ }^{\circ} \mathrm{C}$. After curing, the PDMS was peeled from the mold and inlet holes were punched using a $1 \mathrm{~mm}$ biopsy puncher (Kai Europe $\mathrm{GmbH}$ ) and then bonded onto glass cover slips by air plasma treatment (Plasma Cleaner PDC-002-CE, Harrick Plasma) at 600 mbar for 1 minute. After plasma treatment, the microfluidic chips were kept on a hotplate at $60{ }^{\circ} \mathrm{C}$ for 2 hours. Prior to encapsulation, the microfluidic device was pretreated with a polyelectrolyte solution to provide a hydrophilic coating in the channel between the second junction and the outlet (Fig. S31). First, a 1:3 (v/v) mixture of $37 \%(\mathrm{w} / \mathrm{v}) \mathrm{HCl}$ and $50 \%(\mathrm{w} / \mathrm{v})$ $\mathrm{H}_{2} \mathrm{O}_{2}$ was flushed through from the outlet towards the outer solution inlet by vacuum for 5 minutes to oxidize the channel walls. After washing with water, a $5 \%(\mathrm{w} / \mathrm{v})$ solution of positive polyelectrolyte poly(diallyldimethylammonium chloride) (PDADMAC, $1.04 \mathrm{~g} / \mathrm{ml}, 1.375 \mathrm{n} 20 / \mathrm{D}$ ) was flushed through for 10 minutes. After another water wash, a $2 \%(\mathrm{w} / \mathrm{v})$ solution of negative polyelectrolyte poly(4-styrenesulfonic acid) (PSS, MW 75000) was flushed for 5 minutes to form a hydrophilic coating. This was followed by a final water wash. Pretreated microfluidic chips were used on the same day of treatment.

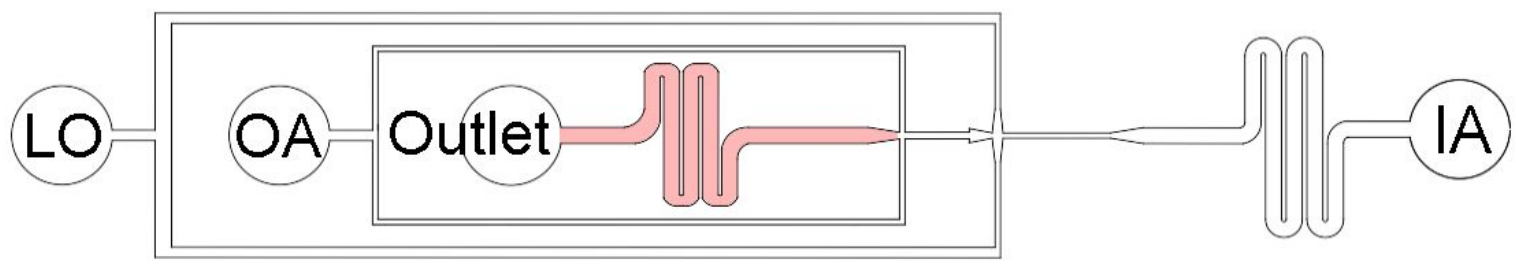

Figure S31. Pretreatment of the microfluidic chip was undertaken by flushing cleaning and coating solutions from the outlet towards the outer aqueous solution inlet (OA) (marked by red arrows) by vacuum. This provides a hydrophilic polyelectrolyte coating in the outlet channel (pink shaded area) to prevent the double-emulsions wetting on the channel walls. IA: inner aqueous solution inlet. LO: lipid oil phase inlet. 


\section{Encapsulation of CFES in liposomes using microfluidics}

Before encapsulation, the osmolarity of the inner PURExpress CFES solution was measured using a freezing point osmometer (Osmomat 3000, Gonotec) calibrated with water, $300 \mathrm{mOsmol} / \mathrm{kg}$, and $2000 \mathrm{mOsmol} / \mathrm{kg}$ $\mathrm{NaCl}$ solution standards. The outer aqueous solution was prepared according to Table S22 and then balanced to the same osmolarity of the inner CFES solution using water or a solution of $1.5 \mathrm{M}$ glucose. DFHBI at a final concentration of $10 \mu \mathrm{M}$ was also added into the outer solution for synthetic cells expressing the RNA Broccoli aptamer. The inner CFES solution was prepared according to Table S18. The lipid oil phase was composed of 1Octanol with $6.5 \mathrm{mM}$ of L- $\alpha$-phosphatidylcholine (Egg PC) phospholipids, and $53.3 \mu \mathrm{M}$ of 1,1'-Dioctadecyl3,3,3',3'-Tetramethylindodicarbocyanine, 4-Chlorobenzenesulfonate Salt (DiD) fluorescent dye. To prepare the lipid oil phase, Egg PC (300 $\mu \mathrm{L}$ of $32.5 \mu \mathrm{M}$ in chloroform) and DiD dye ( $80 \mu \mathrm{L}$ of $1 \mathrm{mM}$ in chloroform) were mixed together in a glass test tube, dried under flowing nitrogen gas for 5 minutes and dried under vacuum for 30 minutes. The dry lipid film was resuspended in $1.5 \mathrm{ml} 1$-octanol, and incubated at $37{ }^{\circ} \mathrm{C}$ in a sonication bath (Sonorex sonicator bath) for 1 hour. To generate synthetic cells, the inner CFES, lipid oil phase, and outer buffer solutions were dispensed through the pretreated microfluidic device (Section 10) using three pressure-regulated pumps (Dolomite Mitos P-Pump) at approximate pressures of 70:80:80 mbar, respectively. This resulted in a flow regime where the inner CFES solution was encapsulated into double-emulsions to form liposomes. Flow regimes and double-emulsion formation in the microfluidic device were monitored under brightfield using a Zeiss Andor Axiovert 200M with a 5x/0.15 Plan-Neofluar Ph1 M27 objective and PCO Dimax S4 Monochrome sCMOS highspeed camera. The cells were collected into a microcentrifuge tube, and then placed in channels made from parafilm channels sandwiched between a microscope slide (76x26x1 mm) and cover slip (24x60 mm). The ends of the channels were sealed with Picodent Twinsil Speed silicone to avoid evaporation (Fig. S32). Imaging was done on either a widefield or confocal microscope (Fig. S33).

Table S22. CFES feeding buffer (outer solution) recipe.

\begin{tabular}{|c|c|}
\hline Component & Concentration \\
\hline ATP & $6 \mathrm{mM}$ \\
\hline CTP ${ }_{*}$ & $4 \mathrm{mM}$ \\
\hline UTP $\}^{*}$ & $4 \mathrm{mM}$ \\
\hline GTP & $6 \mathrm{mM}$ \\
\hline Amino acids** & $0.5 \mathrm{mM}$ \\
\hline Spermidine & $1.5 \mathrm{mM}$ \\
\hline DTT & $1.5 \mathrm{mM}$ \\
\hline Folinic Acid & $0.02 \mathrm{mM}$ \\
\hline K-Glu & $280 \mathrm{mM}$ \\
\hline Mg-Glu & $20 \mathrm{mM}$ \\
\hline HEPES & $100 \mathrm{mM}$ \\
\hline Pluronic Acid & $2 \%$ \\
\hline $\mathrm{KOH}$ & $17.65 \mathrm{mM}$ \\
\hline Glucose & $480 \mathrm{mM}$ \\
\hline $\mathrm{pH}$ & 7.6 \\
\hline Osmolarity & $1441 \mathrm{mOsmol} / \mathrm{kg}$ \\
\hline
\end{tabular}

*Stock NTP mix was prepared by combining NTPs, dissolving in water, and titrating with $15 \% \mathrm{KOH}$ to $\mathrm{pH} 7.5$. **Stock amino acid mix was prepared with each amino acid at $0.5 \mathrm{mM}$ at a final $\mathrm{pH}$ of 7.92 .

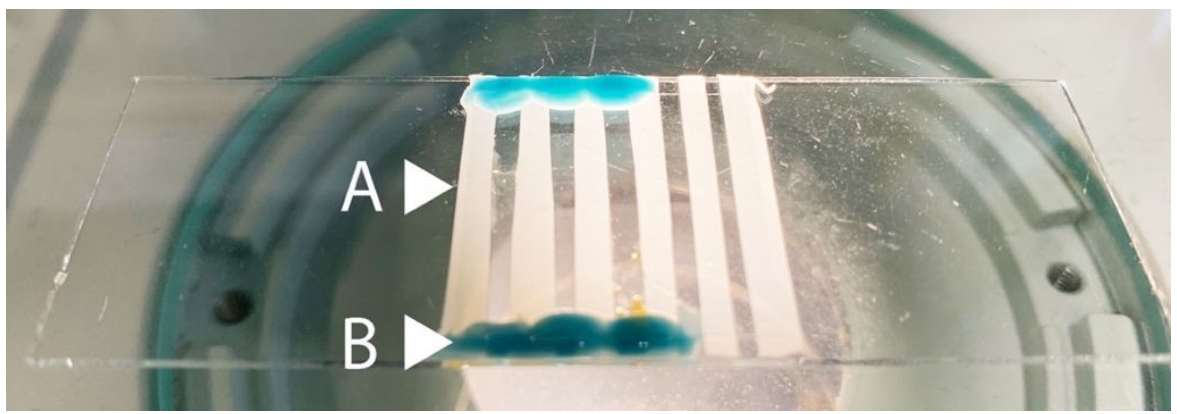

Figure S32. Glass slide setup for confocal microscopy synthetic cell populations. (A) Channels between parafilm strips where synthetic cells are loaded. (B) Twinsil Speed silicone sealing the ends of each channel. 
A)

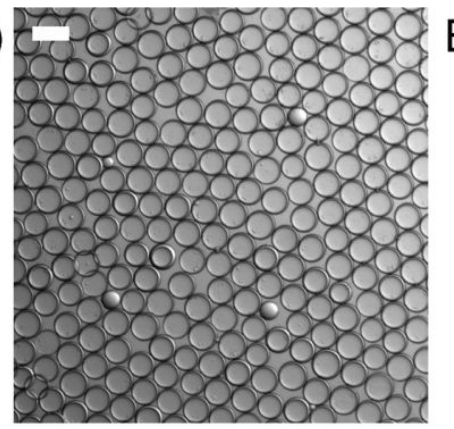

B)

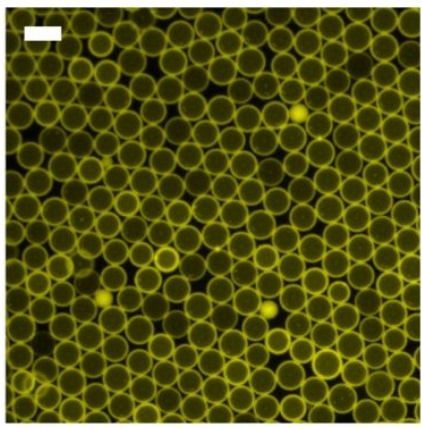

Figure S33. Example of a microfluidic-generated liposome population encapsulating PURExpress CFES. Lipids were composed of Egg PC with DiD dye. (A) Brightfield image and (B) fluorescence image of the same population using a Zeiss Andor Axiovert 200M widefield microscope with a 5x/0.15 Plan-Neofluar Ph1 M27 objective. Fluorescence excitation was at $550 \mathrm{~nm}$ through a ROX filter set (excitation bandpass $575 \pm 15 \mathrm{~nm}$, beam splitter HC BS $596 \mathrm{~nm}$, emission BP $641 \pm 75 \mathrm{~nm}$ ). White scale bars are $100 \mu \mathrm{m}$. 


\section{Inverse emulsion phase transfer method}

Liposomes generated using the bulk inverse emulsion phase transfer method was prepared using a modified protocol as described in [12]. Briefly, 7.5 ul of PURExpress CFES with pEXP5-NT/eGFP plasmid DNA (Table S23) was added into a $1.5 \mathrm{ml}$ microcentrifuge tube with $375 \mathrm{ul}$ of lipid oil phase consisting of $0.39 \mathrm{mM} \mathrm{Egg}$ PC and 1.25 $\mu \mathrm{M}$ Texas Red DHPE in mineral oil. To prepare the lipid oil phase, the POPC ( $36 \mu \mathrm{L}$ of $32.5 \mu \mathrm{M}$ in chloroform) and Texas Red DHPE ( $1 \mu \mathrm{L}$ of $1 \mathrm{mM}$ in chloroform) were mixed together in a glass test tube, dried under flowing nitrogen gas for 5 minutes and in vacuum for 30 minutes, resuspended in $2 \mathrm{~mL}$ mineral oil, and incubated at $37^{\circ} \mathrm{C}$ in a sonication bath (Sonorex sonicator bath) for 1 hour. The mixture was then emulsified by running the tube across a microcentrifuge tube rack ten times. Then, $100 \mu \mathrm{l}$ of the emulsion was gently layered on top of a prepared $100 \mu$ l outer feeding buffer solution (Table S24) $+40 \mu$ lipid oil phase solution in BSA-coated wells of 96-well plate. The plate was centrifuged at $3000 \mathrm{xg}$ for 10 minutes to produce liposomes that settle at the bottom of the plate. The plate was then incubated at $30{ }^{\circ} \mathrm{C}$ overnight (approx. 15 hours) and then imaged using confocal microscopy (LSM 880 with Airyscan, 40X/1.2 C-Apochromat objective). Excitation/detection wavelengths are $488 \mathrm{~nm} / 505-515 \mathrm{~nm}$ for eGFP protein and $594 \mathrm{~nm} / 605-615 \mathrm{~nm}$ for Texas Red DHPE (Fig. S34). Liposomes were segmented manually using Fiji 1.53c [13] and the size and expressed eGFP fluorescence distributions were collected using Python (v3.6) (see Jupyter Notebooks in supplementary files).

Table S23. PURExpress master mix for inverse emulsion

\begin{tabular}{cc}
\hline Component & Volume $(\boldsymbol{\mu l})$ \\
\hline Solution A & 5 \\
Solution B & 3.75 \\
RNAse Inhibitor & 0.25 \\
Sucrose $(400 \mathrm{mM})$ & 1 \\
pEXP5-NT/eGFP $(64 \mathrm{ng} / \mathrm{ul})$ & 0.5 \\
Water & 2 \\
TOTAL & $\mathbf{1 2 . 5}$ \\
\hline
\end{tabular}

Table S24. CFES feeding buffer outer solution recipe for inverse emulsion phase transfer method.

\begin{tabular}{|c|c|}
\hline Component & Concentration \\
\hline ATP & $5 \mathrm{mM}$ \\
\hline CTP & $1.5 \mathrm{mM}$ \\
\hline UTP $\}^{*}$ & $15 \mathrm{mM}$ \\
\hline GTP & $3 \mathrm{mM}$ \\
\hline Amino acids** & $0.5 \mathrm{mM}$ \\
\hline Spermidine & $1.5 \mathrm{mM}$ \\
\hline DTT & $1.5 \mathrm{mM}$ \\
\hline Folinic Acid & $0.02 \mathrm{mM}$ \\
\hline K-Glu & $280 \mathrm{mM}$ \\
\hline Mg-Glu & $20 \mathrm{mM}$ \\
\hline HEPES & $150 \mathrm{mM}$ \\
\hline Glucose & $200 \mathrm{mM}$ \\
\hline $\mathrm{pH}$ & 7.35 \\
\hline Osmolarity & $1339 \mathrm{mOsmol} / \mathrm{kg}$ \\
\hline
\end{tabular}

*Stock NTP mix was prepared by combining NTPs, dissolving in water, and titrating with $15 \% \mathrm{KOH}$ to $\mathrm{pH} 7.5$. ** Stock amino acid mix was prepared according to Table S21. The concentration of each amino acid is $0.5 \mathrm{mM}$.

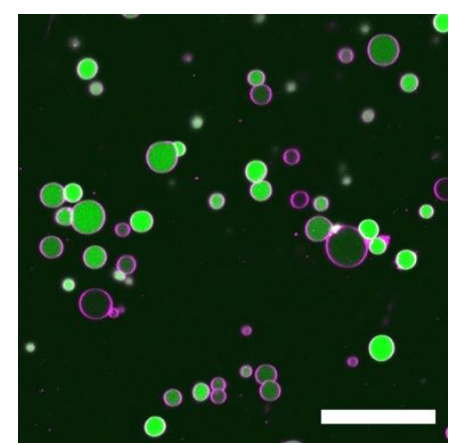

Figure S34. Confocal image composite of inverse emulsion phase transfer generated liposomes encapsulating PURExpress CFES. Liposomes are labeled with Texas Red DHPE in the lipid channel (magenta) and expressed eGFP protein (green) after $\sim 15$ hours incubation at $30^{\circ} \mathrm{C}$. Scale bar is at $100 \mu \mathrm{m}$. 


\section{Image acquisition and analysis}

Here, we briefly describe the steps for image acquisition and analysis of calibration, timeseries, and zstack confocal microscopy data of microfluidic-generated synthetic cell populations.

\section{Image acquisition}

Confocal images of cell free expression encapsulated within lipid vesicles were acquired using an LSM 880 Airyscan inverted laser scanning confocal microscope with a 10X/0.45 Plan-Apochromat M27 objective. The same acquisition settings were used for all microfluidic-generated synthetic cell population experiments. For timelapse imaging, the laser was focused at the equator of the synthetic cells and then imaged at the following excitation/emission wavelengths are $488 \mathrm{~nm} / 499-561 \mathrm{~nm}$ for RNA Broccoli and eGFP protein, $561 \mathrm{~nm} / 579-641$ $\mathrm{nm}$ for mCherry protein, and $633 \mathrm{~nm} / 640-720 \mathrm{~nm}$ for DiD lipid dye every 5 minutes for a total of 12 hours. Zstack images of the samples were taken at the endpoint focused at the top and bottom of the synthetic cells and then divided into 20 intervals.

\section{Software and code}

Image analysis was undertaken in Python (v3.6) using the following packages: Numpy (v1.19.1), Pandas (v1.1.3), Scipy (v1.5.2), Matplotlib (v3.3.1), Pystackreg (v0.2.2) [14], Scikit-image (v0.16.2) [15], and H5py (v3.1.0).

\section{Calibration}

Protein and mRNA standard solutions were prepared on glass slides and imaged under the same experiment confocal microscopy acquisition settings for calibration. A mask of $10 \mu \mathrm{m}$ diameter circles across each image was used to sample each image and obtain fluorescence measurements for the confocal calibration curve (Fig. S35).

\section{Image intensity correction (planar shading correction)}

We observed an uneven and tilted illumination of the samples across the images. Low magnification objectives, such as the 20x objective which we used for our experiments, are sensitive to tilting errors because of their large field of views. Tilting was accounted for by using a planar shading correction [16]. This was done by fitting the single cell fluorescence data points or regions of interest (ROIs) in their respective coordinates to an equation of a plane and then tilting the plane back to a flat horizontal fixed at the origin. This method of correction was applied individually to all confocal images used in this study including the confocal images for calibration. To illustrate, Figs. S36 shows the uneven illumination in an image and the method of correction. Using the grid mask (Fig. S35) for bulk solution images or cell masks (e.g. Fig. S40) for synthetic cell population images, RFU values of the ROIs were plot along the $\mathrm{x}$ and $\mathrm{y}$-axes and then corrected to have an average RFU values to be constant across the plane (Fig. S37).

\section{Analysis of Timelapse images}

Image stacks from timeseries confocal microscopy experiments were first registered to align the stack of each channel to its first image (Fig. S38). The last registered image on the lipid channel stack was then used as a reference image to segment cells of a synthetic cell population throughout the timeseries. A gaussian filter was used to smooth the reference image before thresholding and filling in small holes. The binary image was then labelled for each individual object and filtered by size and circularity to get only the synthetic cells. Cells with defects (e.g. oil drops or shadows from debris) were manually checked and removed. Cells near the edge of the image frame were also avoided (Fig. S39) to ensure only complete vesicles were analyzed. Fluorescence measurements of each labeled synthetic cell were collected from a $10 \mu \mathrm{m}$ diameter circle in the center of each cell (Fig. S40). Single cell fluorescence timeseries measurements for each cell population were saved in csv files.

\section{Z-stack images}

A maximum projection of the lipid channel along the z-axis from z-stack endpoint images of the timeseries confocal microscopy experiments were used as the reference image for cell segmentation. The same workflow of gaussian filter $>$ thresholding $>$ filling $>$ labelling $>$ manual cleaning $>$ filtering by circularity and size was then done on the reference image to segment the synthetic cells (Fig. S41). Cell sizes were obtained from the equivalent diameters of the segmented cells. Fluorescence measurements of each labeled synthetic cell was collected from the maximum value along the z-axis of a $10 \mu \mathrm{m}$ diameter circle in the center of each cell. Single cell fluorescence measurements and sizes for each cell population were saved in csv files. 


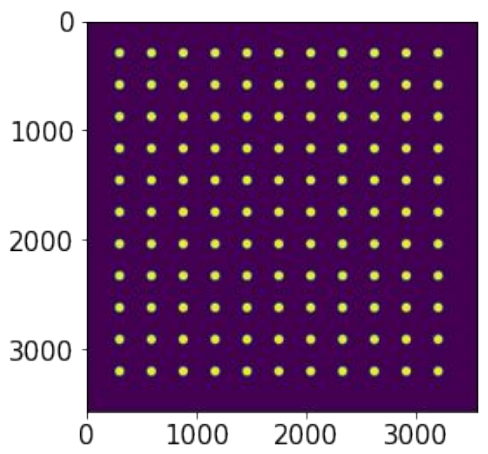

Figure S35. Example mask of $10 \mu \mathrm{m}$ diameter circles in a grid used to collect fluorescence measurements in standard calibration images.
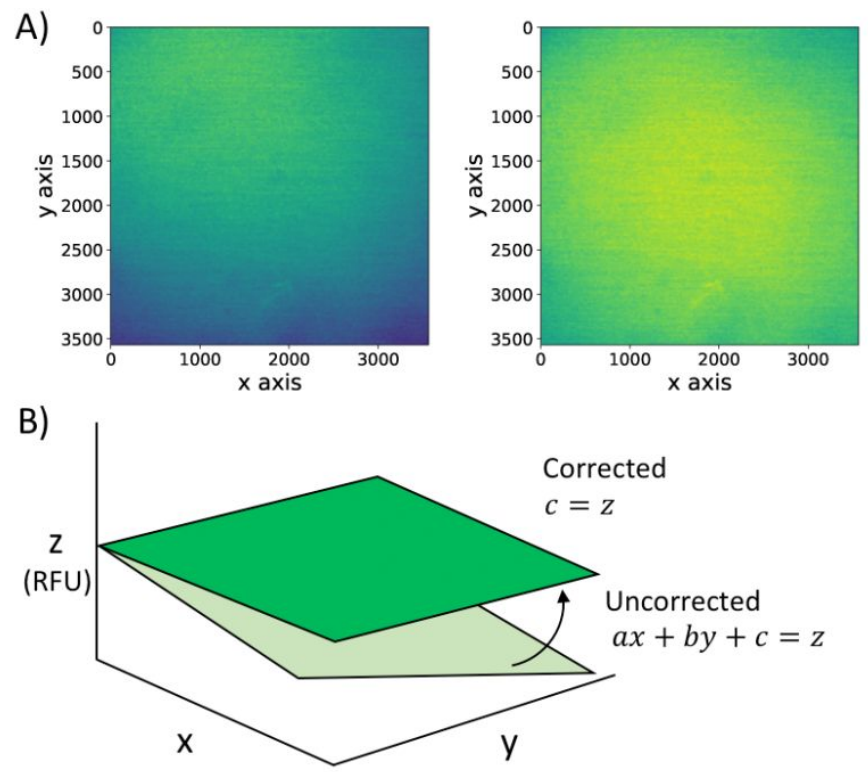

Figure S36. Illustration of image analysis correction for uneven and tilted illumination. (A) Example of confocal image of a bulk sample of eGFP protein with uneven and tilted illumination before (left) and after (right) planar shading correction. Images are gaussian filtered to more distinctly show gradients. (B) Fluorescence values of single cells across the plane follow a gradient due to uneven and tilted illumination. This is corrected by enforcing the average fluorescence signal to be constant across the plane. To obtain the corrected data, fluorescence values were subtracted by $(a x+b y)$ from the plane fit of uncorrected data.
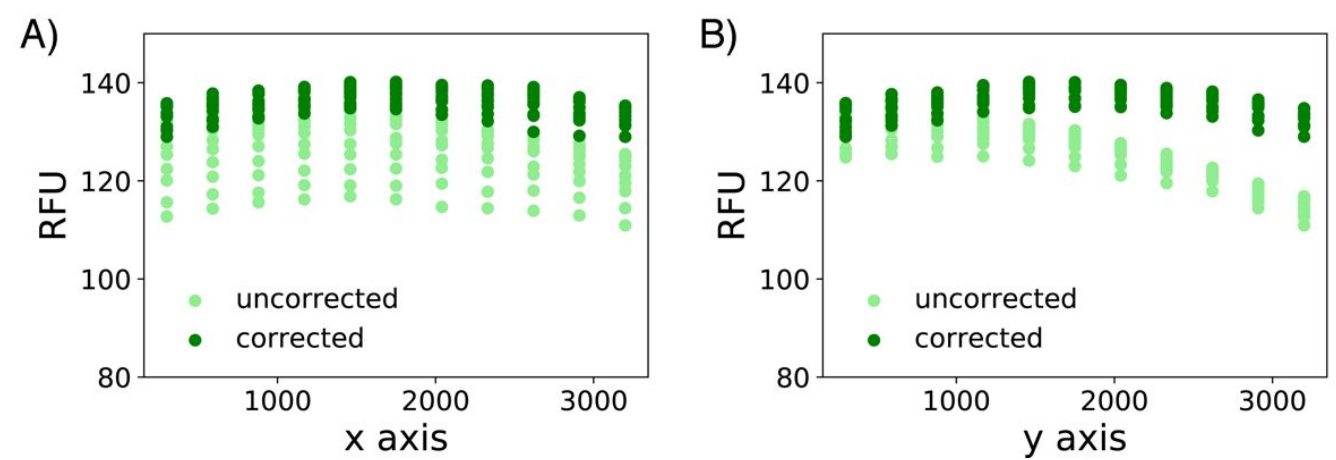

Figure S37. Example dataset from a confocal image with respect to the (A) $x$ and (B) $y$-axes before and after correction from uneven illumination. 

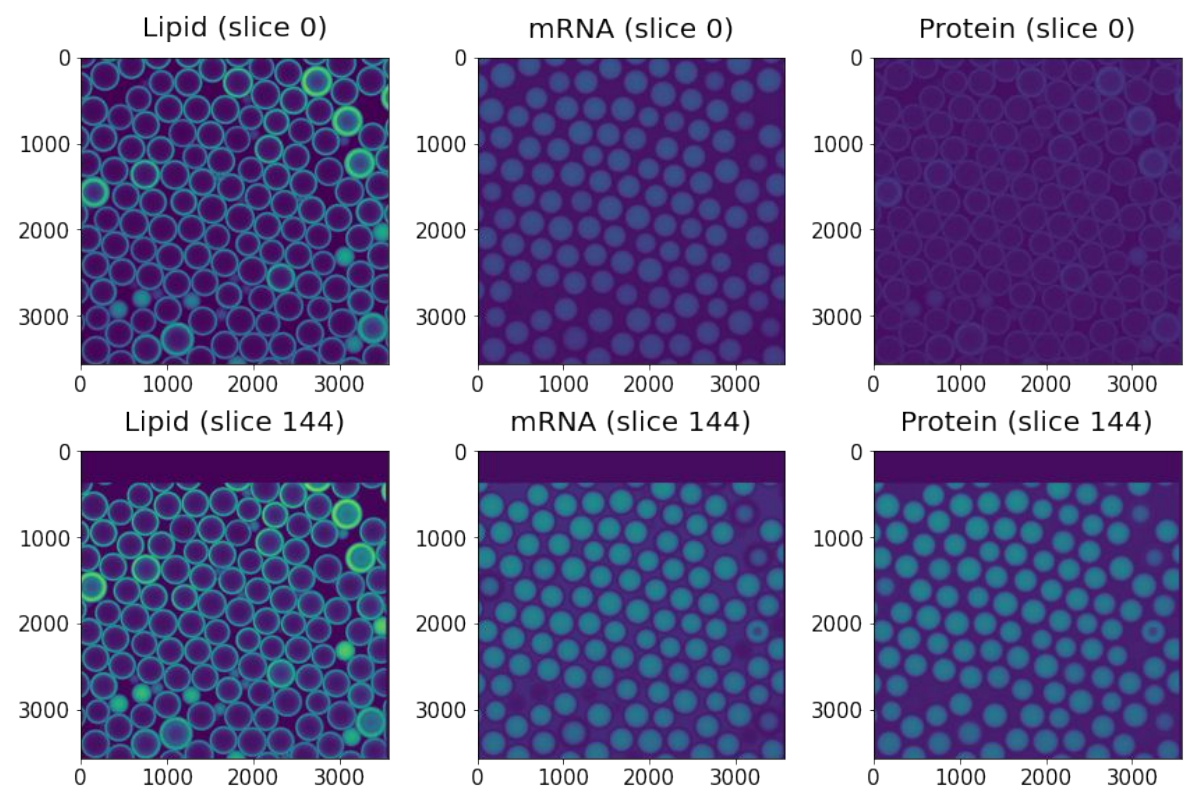

Figure S38. Example image registration of timeseries stacks. The three channels (lipid, mRNA, and protein) for the first (slice 0 ) and last (slice 144) image in the timeseries after registration using Pystackreg. X and Y axes are given in pixel units.
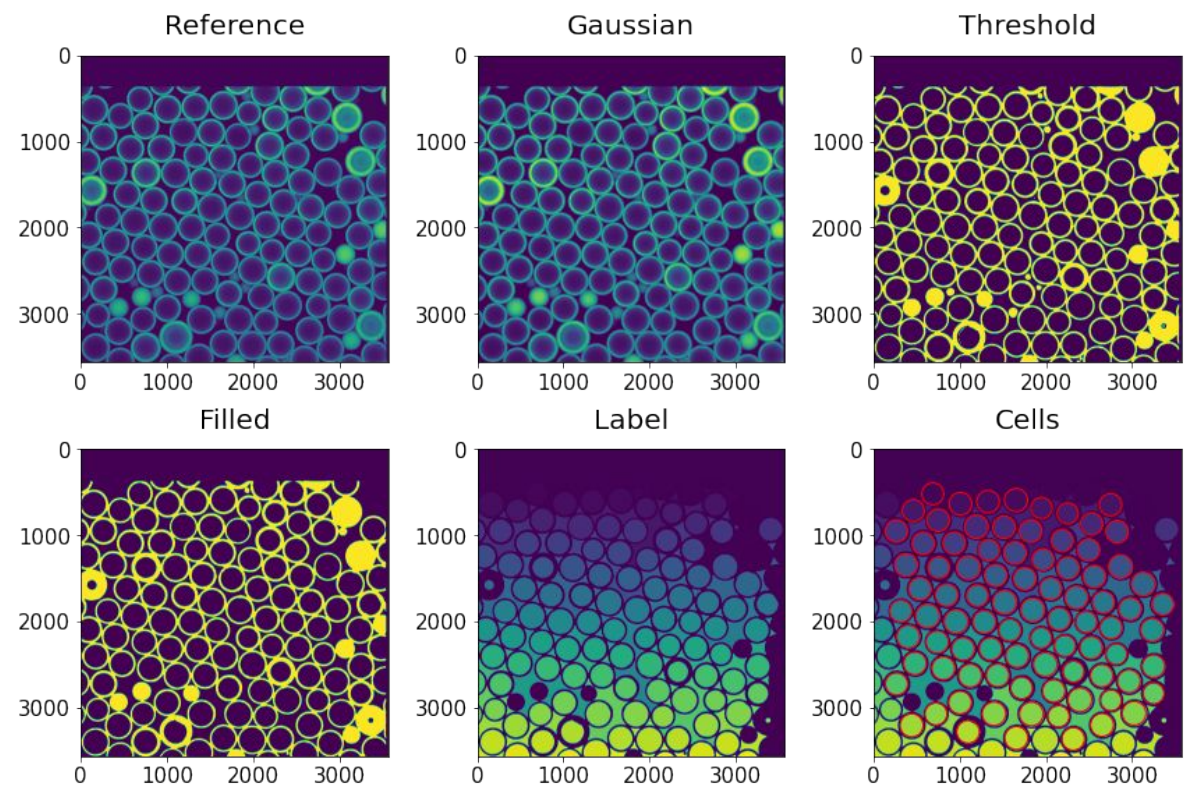

Figure S39. Example image segmentation of a reference image in the lipid channel. From left to right: reference lipid image, gaussian filter, convert to binary by thresholding, filling in small holes, labelling objects, and filtering cells by shape and size (labeled in red circles).

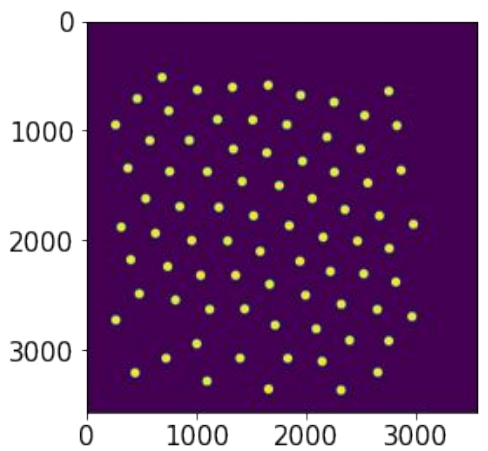

Figure S40. Example mask of $10 \mu \mathrm{m}$ diameter circles from the cell segmentation result in Fig. S39. 

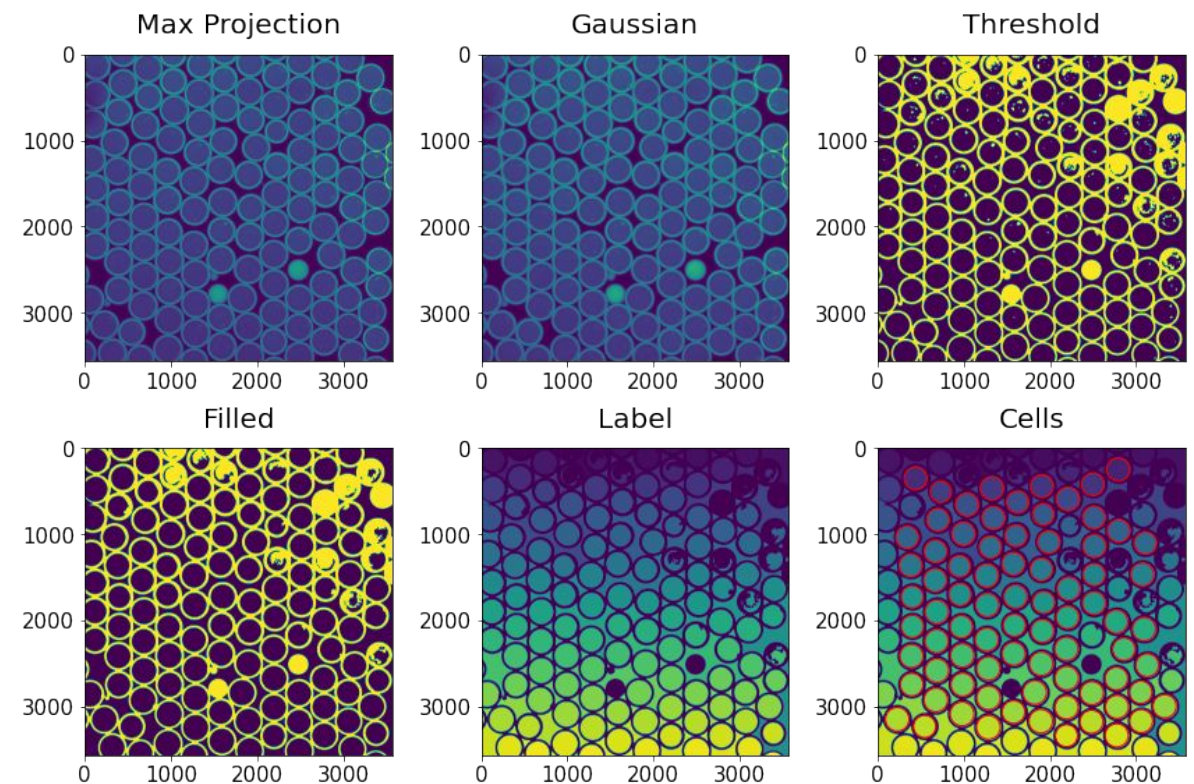

Figure S41. Example image segmentation of a z-stack. From left to right: maximum projection along z-axis, gaussian filter, convert to binary by thresholding, filling in small holes, labelling objects, and filtering cells by shape and size (labeled in red circles). 


\section{Gene expression dynamics in synthetic cell populations}

Synthetic cell populations with varying DNA plasmid concentrations were prepared using a PURExpress CFES inner solution and feeding outer buffer solution. The lipid oil phase was composed of 1-Octanol with $6.5 \mathrm{mM}$ Egg PC, and 53.3 $\mu \mathrm{M}$ DiD dye. Inner solutions were prepared according to Table S18 with different pEXP5$\mathrm{NT} / 6 \mathrm{xHis}$ mCherry F30-2xdBroccoli plasmid DNA concentrations $(1.75,3.5$, and $7.0 \mathrm{nM})$. Outer solution was prepared according to Table S25. Osmolarities of the inner and outer solutions were $1330 \mathrm{mOsmol} / \mathrm{kg}$ and 1334 $\mathrm{mOsmol} / \mathrm{kg}$, respectively. The inner solutions were encapsulated into liposomes using double-emulsion microfluidics (Section 11). The synthetic cell populations were then loaded into channels of the prepared microscope slides for confocal timelapse imaging at $30{ }^{\circ} \mathrm{C}$ incubation for 12 hours (Figs. S42-44). Sample preparation typically took approximately $0.5-1$ hours after adding the plasmid DNA in the PURExpress CFES reaction mix. The CFES was kept on ice during sample preparation until microfluidic encapsulation. For this reason, the initial delay of protein signal is not as evident in the synthetic cell populations as compared to the bulk CFES reactions. Histograms of endpoint expression from z-stack confocal images of the synthetic cell populations are shown in Fig. S47. To demonstrate the reproducibility of the method, endpoint measurements of synthetic cell population experiments from different batches with different DNA concentrations are collated in Fig. S48A. In the case of synthetic cell populations at $3.5 \mathrm{nM}$ pEXP5-NT/6xHis mCherry F30-2xdBroccoli plasmid DNA (boxed in red), a total of 4 different batches results in a batch mean and standard deviation of $1745.4 \mathrm{nM} \pm 183.1 \mathrm{nM}(\mathrm{CV}$ $0.10)$. Gene expression dynamics at this DNA concentration showed batch-wise reproducibility with maximum protein expression rates at $472.6 \pm 85.3 \mathrm{nM} / \mathrm{hr}$ (CV 0.16) (Fig. S48B). Timeseries data of the population mean for each DNA concentration in the synthetic cell populations were used for parameter estimation, profile likelihood calculations (Fig. S49), and confidence intervals (Table S26).

Table S25. Feeding outer solution for DNA titration in synthetic cell populations.

\begin{tabular}{cc}
\hline Component & Volume $(\boldsymbol{\mu l})$ \\
\hline Stock feeding solution (Table S22) & 1000 \\
DFHBI $(4 \mathrm{mM})$ & 2.5 \\
Water & 88 \\
\hline TOTAL & $\mathbf{1 0 9 0 . 5}$ \\
\hline
\end{tabular}
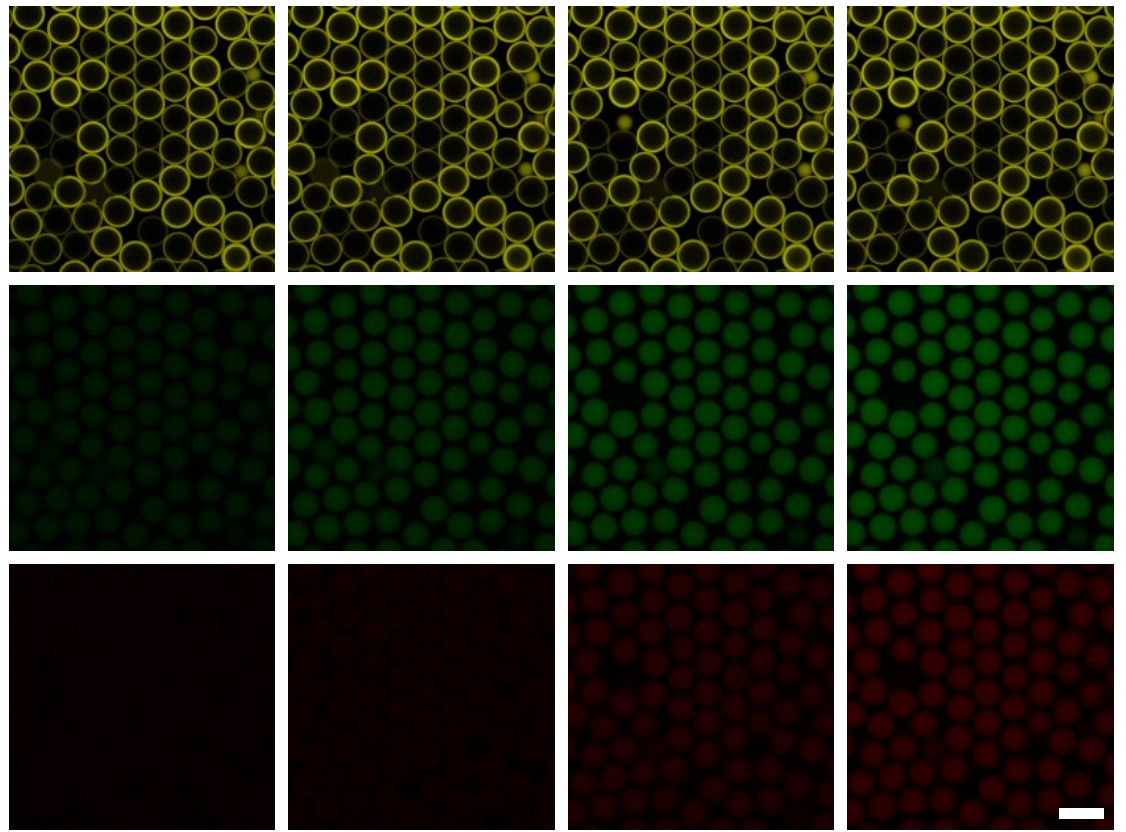

Figure S42. Timeseries images of synthetic cell population with $1.75 \mathrm{nM}$ pEXP5-NT/6xHis mCherry F30 2xdBroccoli plasmid DNA. Top row (yellow) shows the lipid channel, middle row (green) shows the RNA Broccoli channel, and bottom row (red) mCherry protein channel. Images are at $0,1,2$, and 3-hour timepoints. Scale bar is $100 \mu \mathrm{m}$. 

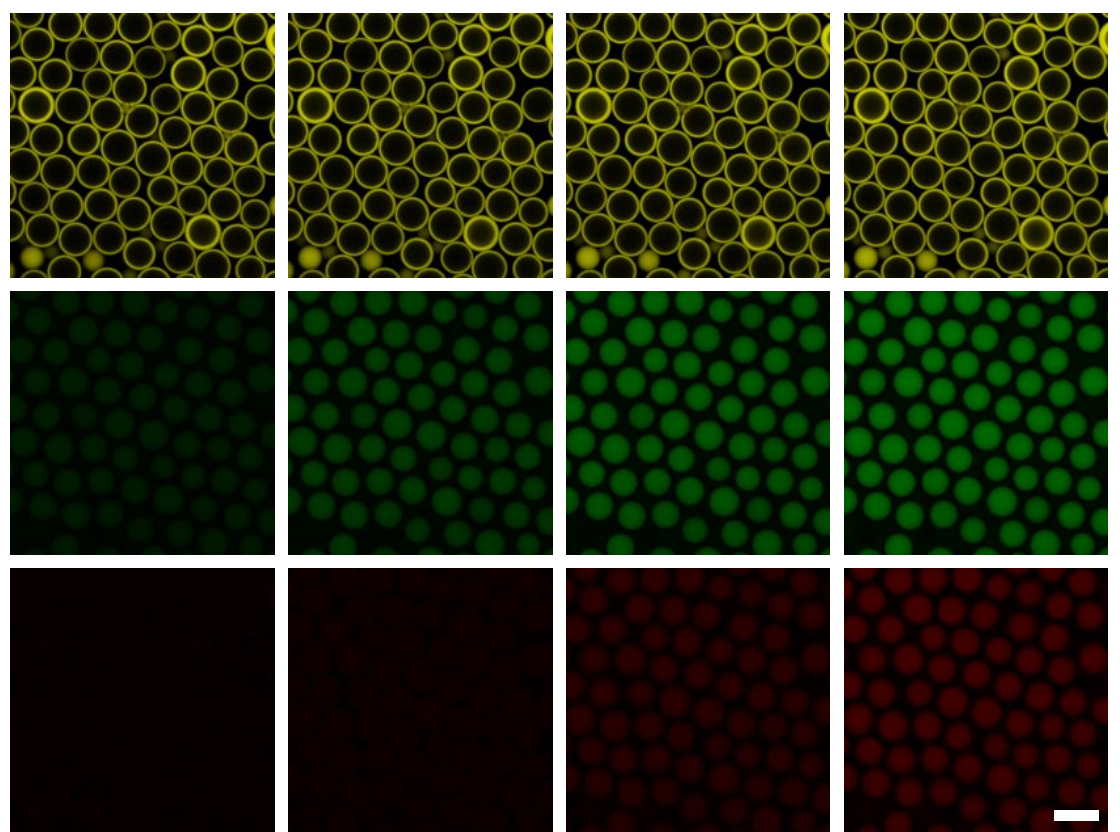

Figure S43. Timeseries images of synthetic cell population with $3.5 \mathrm{nM}$ pEXP5-NT/6xHis mCherry F30 2xdBroccoli plasmid DNA. Top row (yellow) shows the lipid channel, middle row (green) shows the RNA Broccoli channel, and bottom row (red) mCherry protein channel. Images are at $0,1,2$, and 3-hour timepoints. Scale bar is $100 \mu \mathrm{m}$.
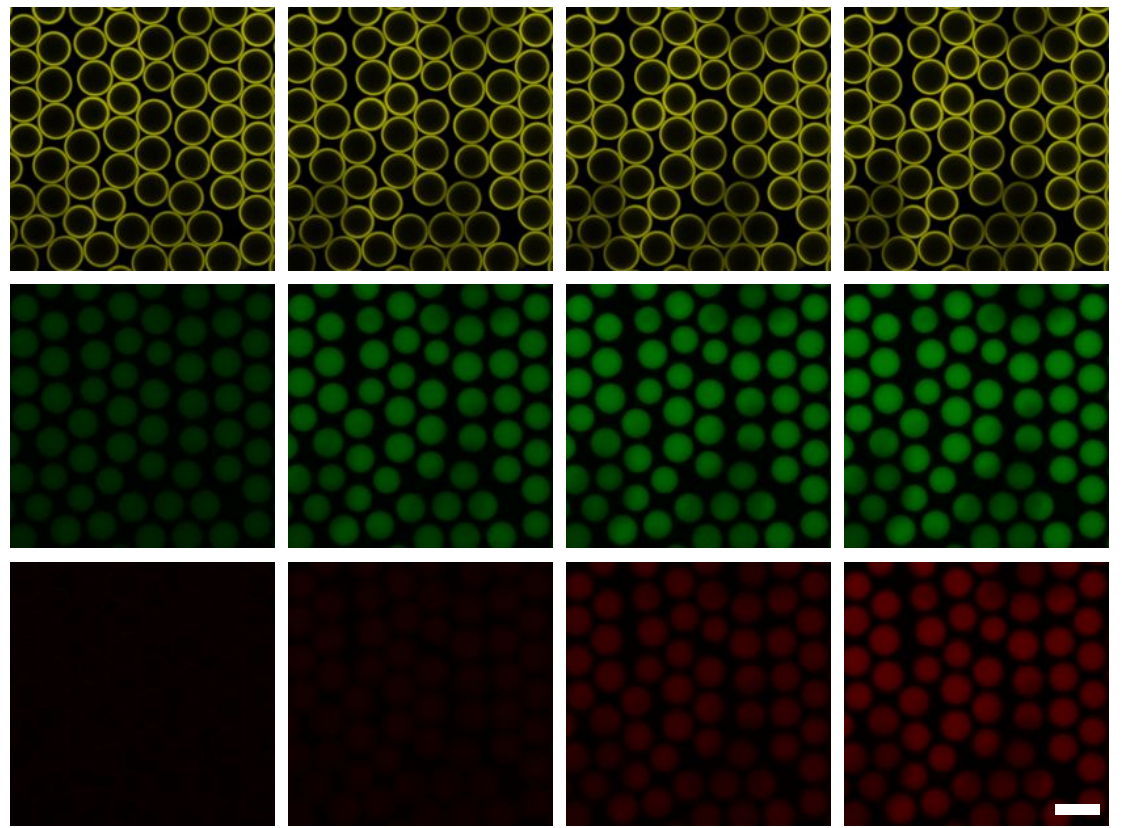

Figure S44. Timeseries images of synthetic cell population with $7 \mathrm{nM}$ pEXP5-NT/6xHis mCherry F30 2xdBroccoli plasmid DNA. Top row (yellow) shows the lipid channel, middle row (green) shows the RNA Broccoli channel, and bottom row (red) mCherry protein channel. Images are at 0,1,2, and 3-hour timepoints. Scale bar is at $100 \mu \mathrm{m}$. 

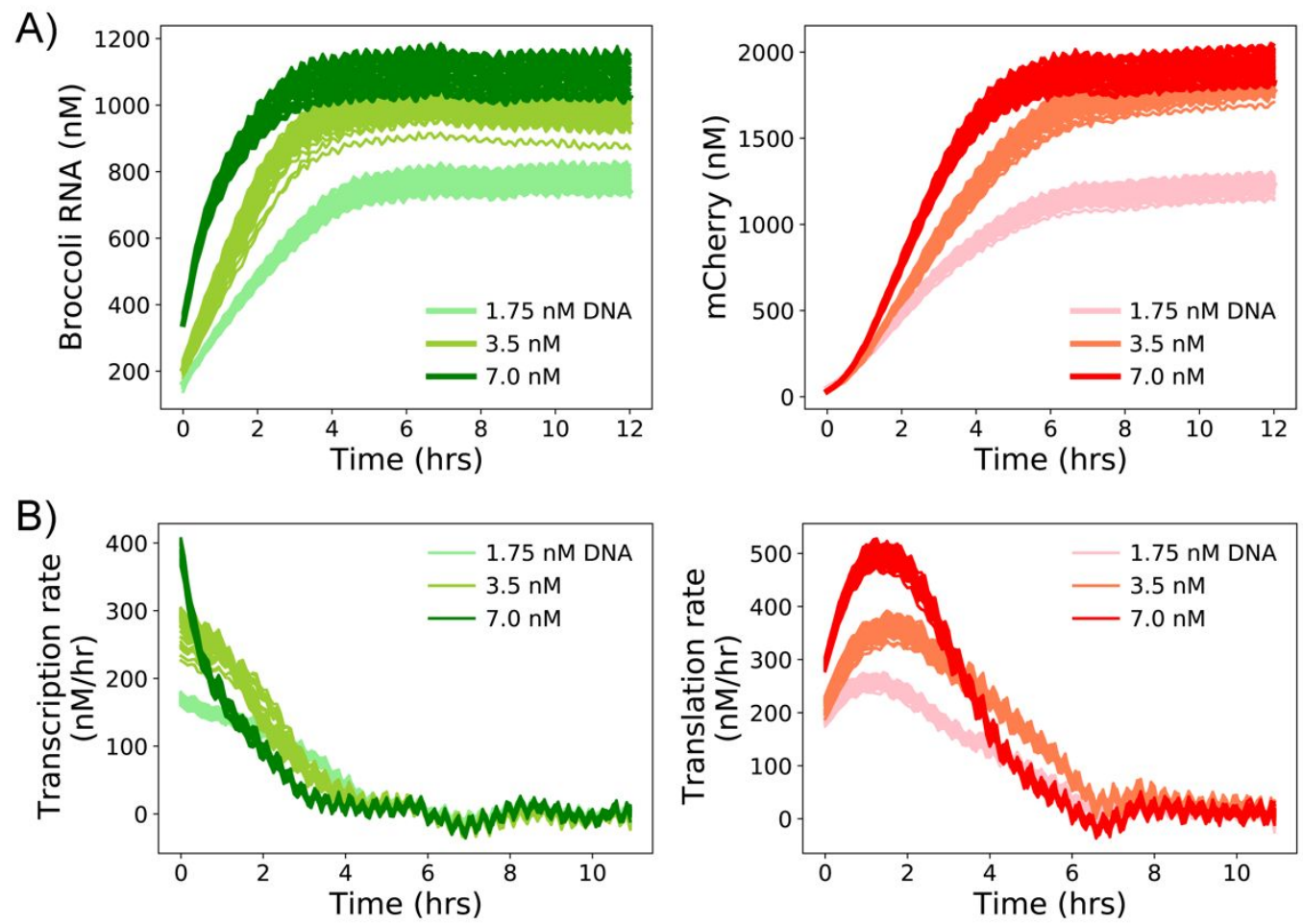

Figure S45. Timeseries of synthetic cell populations with varying DNA concentrations. (A) RNA Broccoli (green) and mCherry protein (red) concentrations over time for 1.75, 3, and $7 \mathrm{nM}$ pEXP5-NT/6xHis mCherry F30 2xdBroccoli plasmid DNA. (B) Rates of transcription and translation calculated from (A) using a rolling window average of 1 hour.
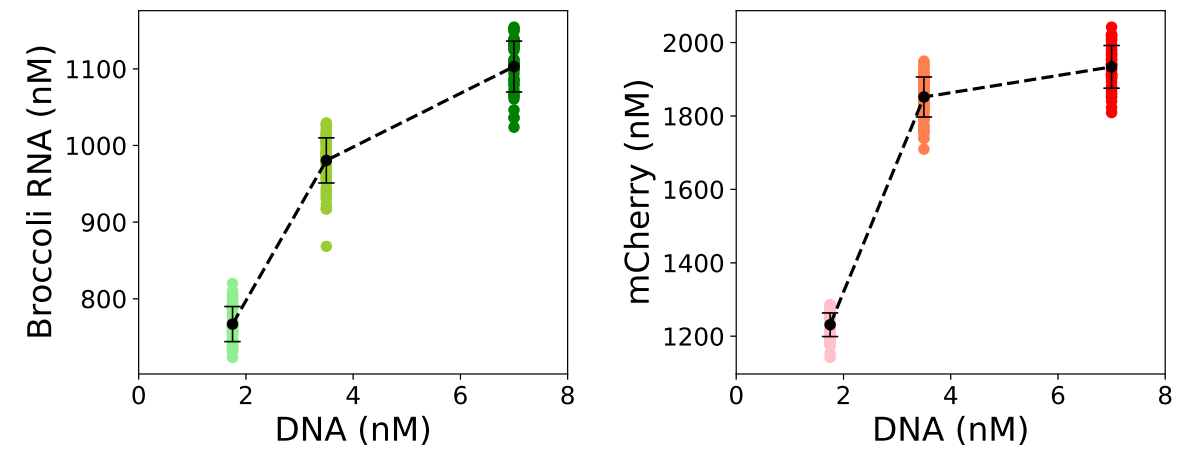

Figure S46. Endpoint RNA (green) and protein (red) concentrations of synthetic cell populations at varying DNA concentrations. Each dot is a single synthetic cell from endpoint. Black dots with error bars are mean and standard deviation of the populations. 

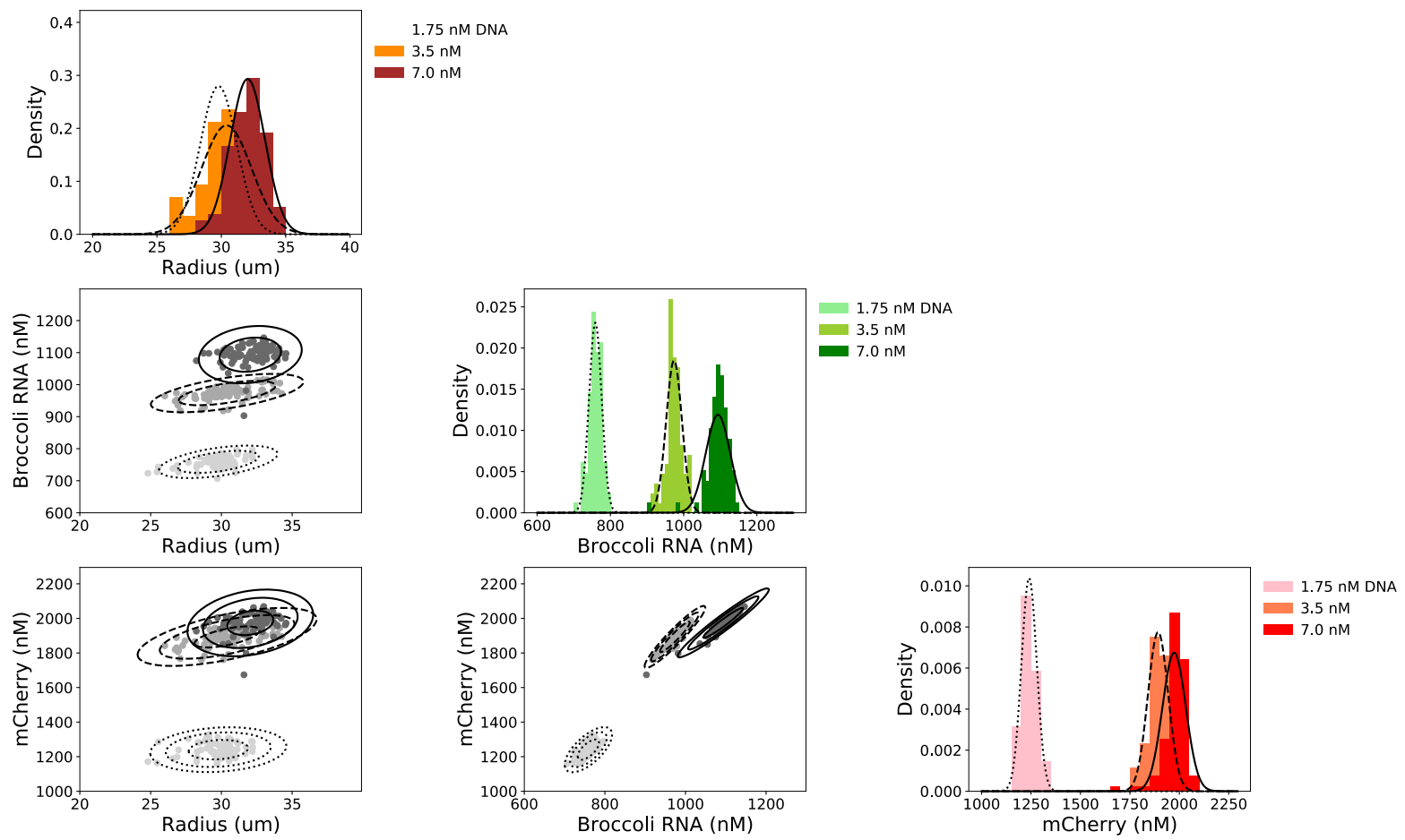

Figure S47. Distributions and correlations of radius, RNA, and protein concentrations in synthetic cell populations. Data is obtained from maximum values of z-stack images at endpoint after $30{ }^{\circ} \mathrm{C}$ incubation for 12 hours. Histograms are fit to univariate normal distributions and correlations are fit to bivariate normal distributions. Total number of cells analyzed are 82,85 , and 78 for populations 1,2 , and 3 , respectively.
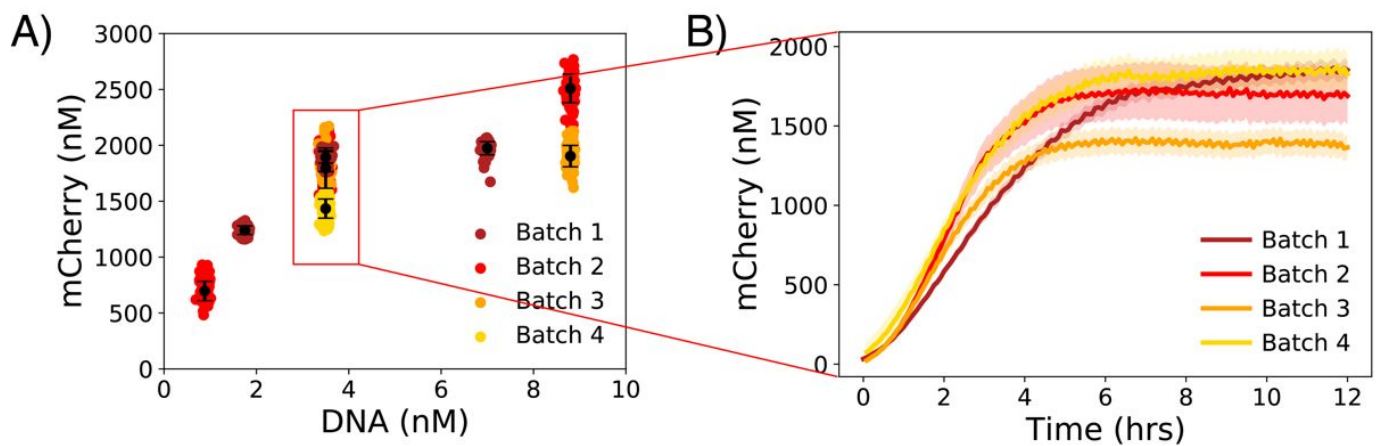

Figure S48. Batch variability of the synthetic cell populations. (A) Endpoint concentrations of synthetic cell populations at varying pEXP5-NT/6xHis mCherry F30 2xd-Broccoli plasmid DNA concentrations from different batches. Batches are plotted in different colors (each dot is a single synthetic cell) and means the experiment was performed on a different day with a new set of inner CFES, feeding outer buffer, and lipid phase solutions. Black dots with error bars are mean and standard deviation of the populations. (B) Dynamics of the different batches of synthetic cell populations with $3.5 \mathrm{nM}$ pEXP5-NT/6xHis mCherry F30 2xd-Broccoli plasmid DNA. 

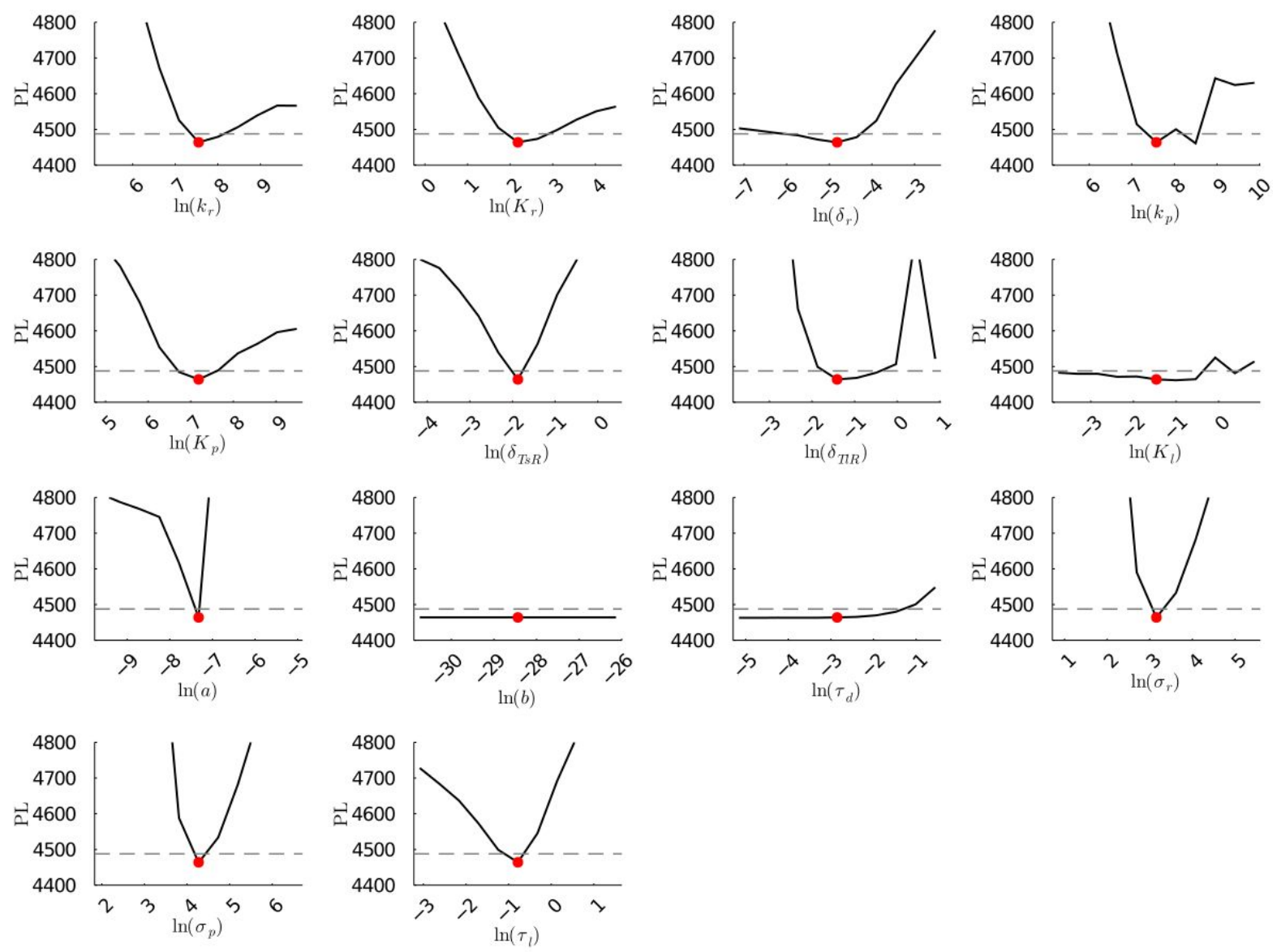

Figure S49. Profile likelihoods of parameters from model 2 using synthetic population experiment data. Each plot corresponds to a parameter in the model and additional fitting parameters $\left(\sigma_{r}, \sigma_{p}\right.$, and $\left.\tau_{d}\right)$. The y-axis of each plot shows the minimized negative log-likelihood of the model with respect to all parameters except for the parameter given its value in the $\mathrm{x}$-axis. The red dot shows the optimized parameter set with the minimum negative log likelihood. The dashed grey line is the $95 \%$ significance threshold line. The intersections of the significance threshold line and profile likelihood are the likelihood-based confidence intervals of the optimized parameter.

Table S26. Parameter estimates and 95\% likelihood-based confidence intervals of model 2 using synthetic cell population data.

\begin{tabular}{cc|cc}
\hline Parameter & $\hat{\boldsymbol{\theta}}$ & \multicolumn{2}{|c}{ 95\% CI } \\
\hline$k_{r}$ & 1899 & 1631 & 3537 \\
$K_{r}$ & 8.86 & 6.98 & 18.66 \\
$\delta_{r}$ & 0.0081 & 0.00239 & 0.0143 \\
$k_{p}$ & 1954 & 1617 & 2696 \\
\hline$K_{p}$ & 1319 & 819 & 2038 \\
$k_{m a t}$ & 2.15 & \multicolumn{2}{|c}{$( \pm 0.12)$} \\
\hline$\delta_{T s R}$ & 0.154 & 0.136 & 0.175 \\
$\delta_{T l R}$ & 0.244 & 0.184 & 0.684 \\
\hline$K_{l}$ & 0.232 & $-\infty$ & 0.713 \\
\hline$a$ & $6.60 \mathrm{E}-4$ & $6.21 \mathrm{E}-4$ & $6.74 \mathrm{E}-4$ \\
\hline$b$ & $4.46 \mathrm{E}-13$ & $-\infty$ & $+\infty$ \\
\hline$\tau_{d}$ & 0.0576 & $-\infty$ & 0.279 \\
\hline$\sigma_{r}$ & 23.48 & 21.83 & 28.25 \\
\hline$\sigma_{p}$ & 71.58 & 66.40 & 86.09 \\
\hline$\tau_{l}$ & 0.457 & 0.342 & 0.535 \\
\hline
\end{tabular}




\section{Two plasmids in a synthetic cell population}

By encapsulating two plasmids, pEXP5-NT/6xHis mCherry and pEXP5-NT/6xHis eGFP, a synthetic cell population can express both mCherry and eGFP protein. Inner and outer solutions were made according to Tables S27-28 and then encapsulated into liposomes using double-emulsion microfluidics. The lipid oil phase was composed of 1-Octanol with $6.5 \mathrm{mM}$ Egg PC, and 53.3 $\mu \mathrm{M}$ DiD dye. The synthetic cell populations were then loaded into channels of the prepared microscope slides for confocal timelapse imaging at $30{ }^{\circ} \mathrm{C}$ incubation for 12 hours (Figs. S50-51). Z-stack confocal images of the synthetic cell populations were acquired at endpoint (Fig. S52).

Table S27. PURExpress inner solution for DNA titration in synthetic cell populations.

\begin{tabular}{cccc}
\hline Component & $\begin{array}{c}\text { eGFP \& } \\
\text { mCherry }(\boldsymbol{\mu l})\end{array}$ & $\begin{array}{c}\text { eGFP } \\
\text { only }(\boldsymbol{\mu l})\end{array}$ & $\begin{array}{c}\text { mCherry } \\
\text { only }(\boldsymbol{\mu l})\end{array}$ \\
\hline Solution A & 50 & 50 & 50 \\
Solution B & 37.5 & 37.5 & 37.5 \\
RNAse Inhibitor & 2.5 & 2.5 & 2.5 \\
Sucrose $(1.5 \mathrm{M})$ & 6.67 & 6.67 & 6.67 \\
$\mathrm{pEXP5-NT/6xHis} \mathrm{mCherry}(533 \mathrm{ng} / \mu \mathrm{L})$ & 2.35 & 0 & 2.35 \\
$\mathrm{pEXP5-NT/6xHis} \mathrm{eGFP}(467 \mathrm{ng} / \mu \mathrm{L})$ & 2.68 & 2.68 & 0 \\
Water & 23.3 & 25.65 & 25.98 \\
\hline TOTAL & $\mathbf{1 2 5}$ & $\mathbf{1 2 5}$ & $\mathbf{1 2 5}$ \\
Osmolarity & & $1230 \mathrm{mOsmol} / \mathrm{kg}$ & \\
\hline
\end{tabular}

Table S28. Feeding outer solution for DNA titration in synthetic cell populations.

\begin{tabular}{cc}
\hline Component & Volume $(\boldsymbol{\mu l})$ \\
\hline Stock feeding solution (Table S22) & 1000 \\
Water & 124 \\
\hline TOTAL & $\mathbf{1 1 2 4}$ \\
Osmolarity & $1230 \mathrm{mOsmol} / \mathrm{kg}$ \\
\hline
\end{tabular}
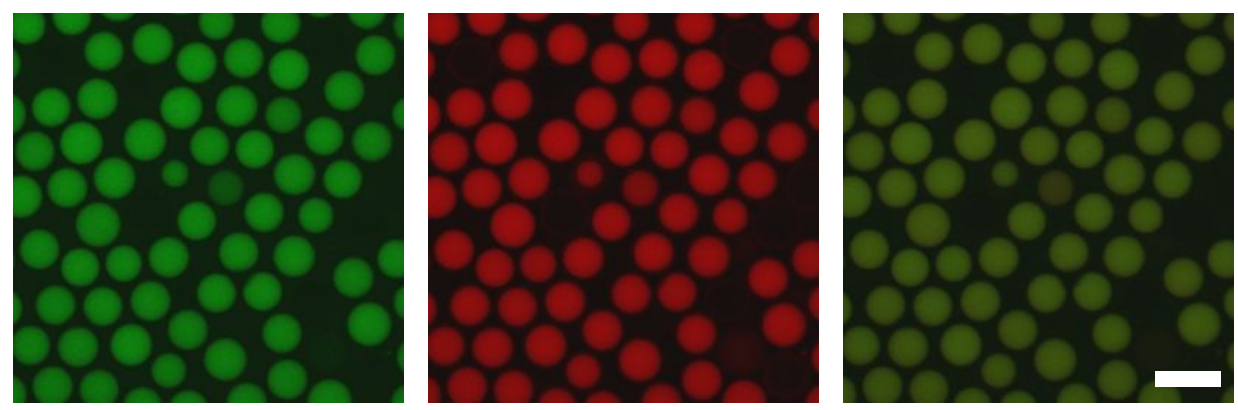

Figure S50. Endpoint confocal image of a cell population containing two plasmids pEXP5-NT/6xHis mCherry and pEXP5-NT/6xHis eGFP plasmids both at $10 \mathrm{ng} / \mu \mathrm{L}$. Left panel (green) shows the eGFP protein channel, the middle panel mCherry protein channel, and the left panel is the merged image of eGFP and mCherry. Scale bar is at $100 \mu \mathrm{m}$.
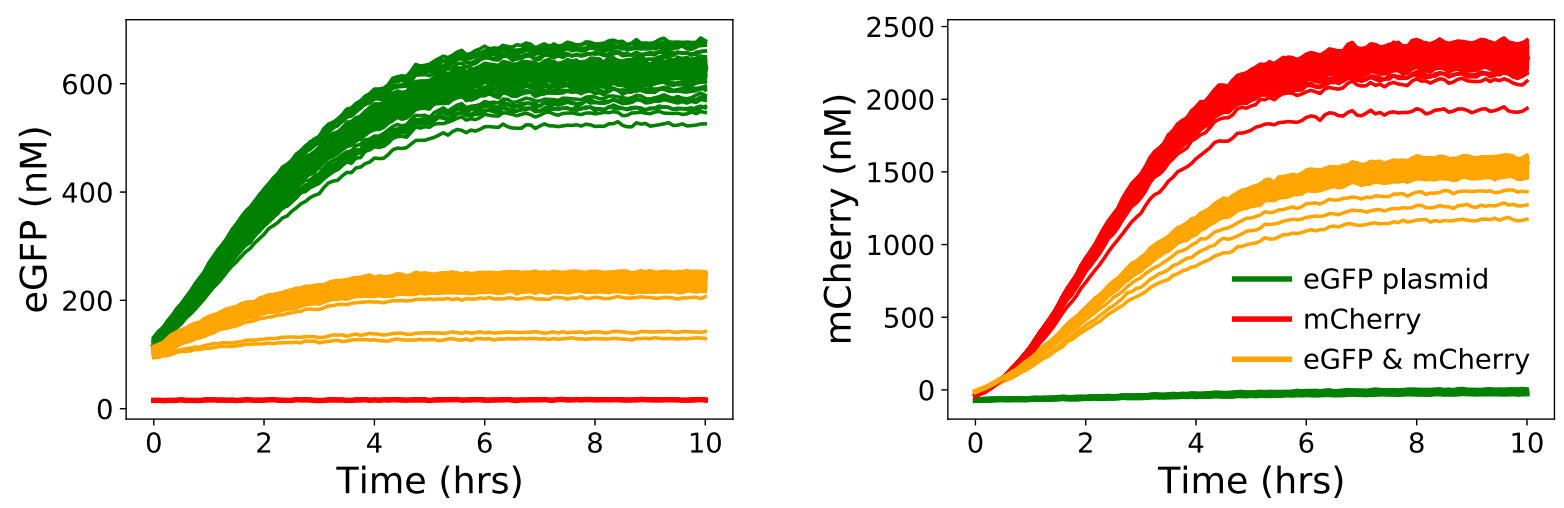
Figure S51. Timeseries of synthetic cell populations with pEXP5-NT/6xHis mCherry (red), pEXP5-NT/6xHis eGFP (green), or both pEXP5-NT/6xHis mCherry and pEXP5-NT/6xHis eGFP plasmids (yellow). Each plasmid is at a concentration of $10 \mathrm{ng} / \mathrm{ul}$.
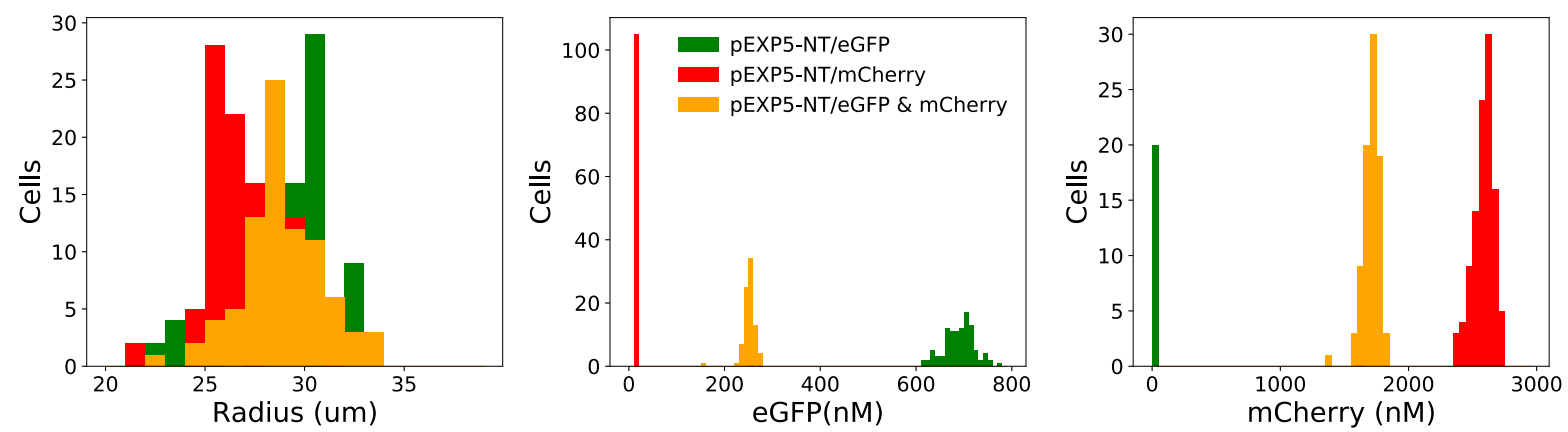

Figure S52. Histograms of endpoint radius, eGFP, and mCherry protein in the synthetic cell populations with pEXP5-NT/6xHis mCherry (red), pEXP5-NT/6xHis eGFP (green), or both pEXP5-NT/6xHis mCherry and pEXP5-NT/6xHis eGFP plasmids (yellow). Each plasmid is at a concentration of $10 \mathrm{ng} / \mathrm{ul}$. Data is obtained from maximum values of $z$-stack images. 


\section{Dilution of CFES outer solution in synthetic cell populations}

To determine if the outer solution conditions in microfluidic-generated synthetic cells significantly affect gene expression rates, synthetic cell populations were prepared with different dilutions of the outer solution composed of PURExpress CFES without DNA. An inner solution was prepared according to Table S18 with $3 \mathrm{nM}$ of pEXP5-NT/6xHis mCherry F30-2xdBroccoli plasmid DNA (final osmolarity $1346 \mathrm{mOsmol} / \mathrm{kg}$ ). The outer solution was also composed of PURExpress CFES, with glucose, pluronic acid, and no DNA (Table S29). To dilute the outer solution, a dilution mix composed of the same concentration of $10 \mu \mathrm{M}$ DFHBI for the Broccoli aptamer, 2\% pluronic acid to stabilize the synthetic cells, and glucose to maintain the same osmolarity of the outer solution (Table S30). Diluting the outer solution with the dilution mix then results in the dilution of all the buffer components except for DFHBI, pluronic acid, and the overall osmolarity. The lipid oil phase was composed of 1Octanol with $6.5 \mathrm{mM}$ Egg PC, and $53.3 \mu \mathrm{M}$ DiD dye. The inner solution was encapsulated into liposomes by double-emulsion microfluidics. Aliquots of the collected liposomes were diluted with the dilution mix to result in $100 \%, 66 \%, 50 \%, 33 \%, 10 \%$ PURExpress CFES outer solutions. This was done by dropping a $10 \mu \mathrm{L}$ aliquot of the synthetic cells onto a microscope slide and then adding the appropriate volume of the dilution mix $(0,5,10$, $20,100 \mu \mathrm{L}$, respectively). The diluted liposomes were then gently mixed by pipetting and then loaded into channels of the prepared microscope slides for confocal timelapse imaging at $30{ }^{\circ} \mathrm{C}$ incubation for 12.5 hours. Despite the inner, outer, and dilution solutions being osmotically balanced, differences in composition between diluted outer solutions and the inner solutions resulted in material flux into the liposomes. This is because osmolarities are indirectly measured and balanced using freezing point depression measurements. As a result, comparing complex solutions of different compositions will deviate from the actual osmolarities [17]. This is observed in larger liposomes or bursting in the diluted samples. mRNA and protein expression were also negatively affected with more dilution of the PURExpress outer solution (Fig. S53-54).

Table S29. PURExpress outer solution.

\begin{tabular}{cc}
\hline Component & Volume $(\boldsymbol{\mu l})$ \\
\hline Solution A & 100 \\
Solution B & 75 \\
RNAse Inhibitor & 5 \\
DFHBI $(500 \mu \mathrm{M})$ & 5 \\
$10 \%$ Pluronic acid & 50 \\
Glucose $(1.5 \mathrm{M})$ & 13.3 \\
Water & 13.2 \\
\hline TOTAL & $\mathbf{2 6 1 . 5}$ \\
Osmolarity & $1363 \mathrm{mOsmol} / \mathrm{kg}$ \\
\hline
\end{tabular}

Table S30. Dilution mix.

\begin{tabular}{cc}
\hline Component & Volume $(\boldsymbol{\mu l})$ \\
\hline Glucose $(1.5 \mathrm{M})$ & 815 \\
DFHBI $(4 \mathrm{mM})$ & 3 \\
$10 \%$ Pluronic acid & 240 \\
Water & 142 \\
\hline TOTAL & $\mathbf{1 2 0 0}$ \\
Osmolarity & $1351 \mathrm{mOsmol} / \mathrm{kg}$ \\
\hline
\end{tabular}


A)
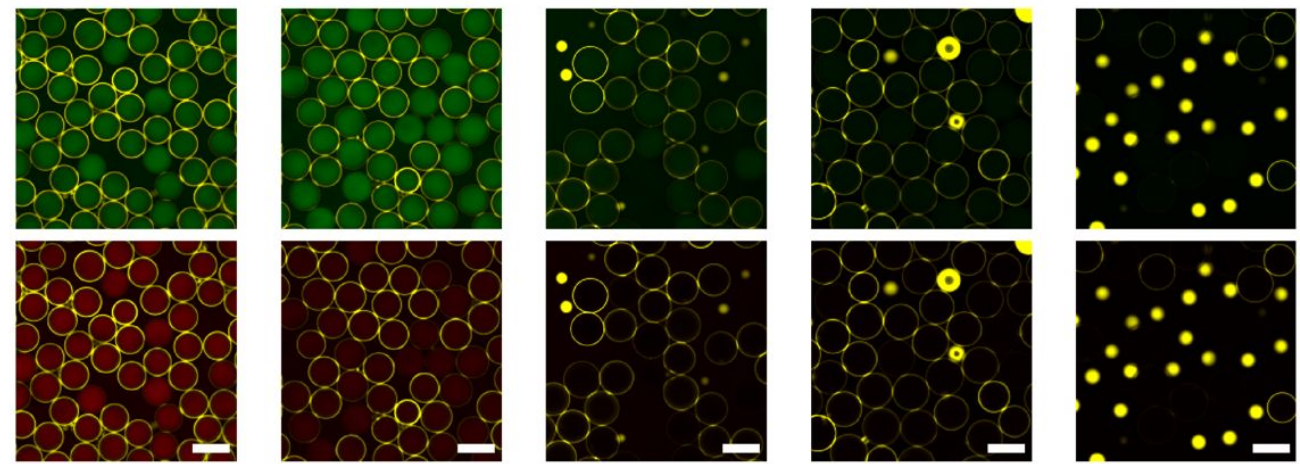

B)
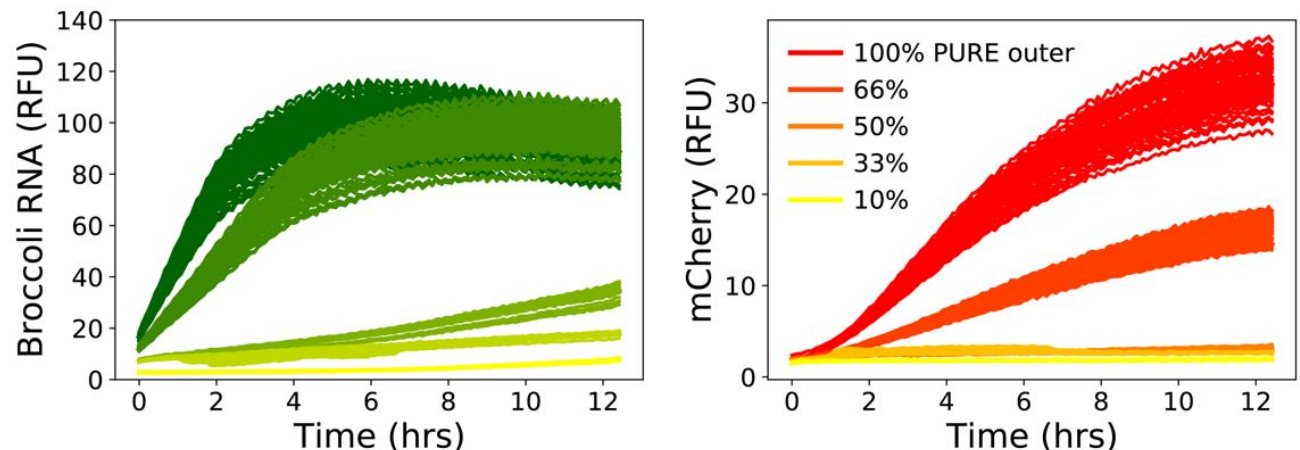

Figure S53. Dilutions of the outer solution in synthetic cell populations. (A) Confocal images of the synthetic cell populations with $3 \mathrm{nM}$ of pEXP5-NT/6xHis mCherry F30-2xdBroccoli plasmid DNA in decreasing percentage of PURExpress CFES within the outer solution $(100 \%, 66 \%, 50 \%, 33 \%$, and $10 \%$ from left to right) at endpoint after $30^{\circ} \mathrm{C}$ incubation for 12.5 hours. Top row is for mRNA Broccoli channel (green) and bottom row is for mCherry protein (red) both overlaid with the lipid DiD dye channel (yellow). (B) Single-cell gene expression dynamics of expressed RNA and protein of the same synthetic cell populations. Total number of cells analyzed are $66,69,17$, 16 , and 17 for $100 \%, 66 \%, 50 \%, 33 \%$, and $10 \%$ PURExpress outer solution populations, respectively. Scale bars are all $100 \mu \mathrm{m}$.
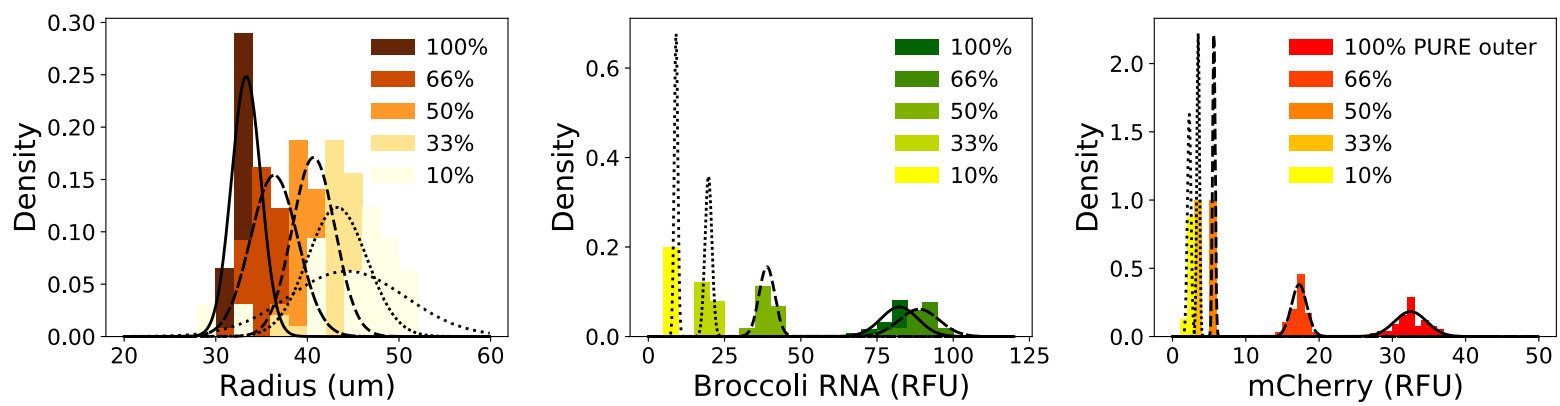

Figure S54. Endpoint histograms of radius, RNA Broccoli, and mCherry protein from confocal z-stack images of the synthetic cell populations at $100 \%, 66 \%, 50 \%, 33 \%$, and $10 \%$ PURExpress outer solution after 12.5 hours of incubation at $30^{\circ} \mathrm{C}$. Total number of cells analyzed are $76,65,32,48$, and 16 for $100 \%, 66 \%, 50 \%, 33 \%$, and $10 \%$ PURExpress outer solution populations, respectively. 


\section{References}

[1] Li M, Green DC, Anderson JLR, Binks BP, Mann S: In vitro gene expression and enzyme catalysis in bio-inorganic protocells. Chem Sci 2011, 2:1739.

[2] Tang T-YD, van Swaay D, DeMello A, Ross Anderson JL, Mann S: In vitro gene expression within membrane-free coacervate protocells. Chem Commun 2015, 51:11429-11432.

[3] Filonov GS, Moon JD, Svensen N, Jaffrey SR: Broccoli: Rapid selection of an RNA mimic of green fluorescent protein by fluorescence-based selection and directed evolution. J Am Chem Soc 2014, 136:16299-16308.

[4] Filonov GS, Kam CW, Song W, Jaffrey SR: In-gel imaging of RNA processing using broccoli reveals optimal aptamer expression strategies. Chem Biol 2015, 22:649-660.

[5] Gross LA, Baird GS, Hoffman RC, Baldridge KK, Tsien RY: The structure of the chromophore within DsRed, a red fluorescent protein from coral. Proc Natl Acad Sci U S A 2000, 97:1199011995.

[6] Garamella J, Marshall R, Rustad M, Noireaux V: The All E. coli TX-TL Toolbox 2.0: A Platform for Cell-Free Synthetic Biology. ACS Synth Biol 2016, 5:344-355.

[7] Stögbauer T, Windhager L, Zimmer R, Rädler JO: Experiment and mathematical modeling of gene expression dynamics in a cell-free system. Integr Biol 2012, 4:494.

[8] Bezanson J, Edelman A, Karpinski S, Shah VB: Julia: A fresh approach to numerical computing. SIAM Rev 2017, 59:65-98.

[9] Raue A, Kreutz C, Maiwald T, Bachmann J, Schilling M, Klingmüller U, Timmer J: Structural and practical identifiability analysis of partially observed dynamical models by exploiting the profile likelihood. Bioinformatics 2009, 25:1923-1929.

[10] Maiwald T, Hass H, Steiert B, Vanlier J, Engesser R, Raue A, Kipkeew F, Bock HH, Kaschek D, Kreutz $\mathrm{C}$, et al.: Driving the model to its limit: Profile likelihood based model reduction. PLoS One 2016, 11:1-18.

[11] Yandrapalli N, Petit J, Bäumchen O, Robinson T: Surfactant-free production of biomimetic giant unilamellar vesicles using PDMS-based microfluidics. Commun Chem 202141 2021, 4:1-10.

[12] Moga A, Yandrapalli N, Dimova R, Robinson T: Optimization of the inverted emulsion method for high-yield production of biomimetic giant unilamellar vesicles. ChemBioChem 2019, doi:10.1002/cbic.201900529.

[13] Schindelin J, Arganda-Carreras I, Frise E, Kaynig V, Longair M, Pietzsch T, Preibisch S, Rueden C, Saalfeld S, Schmid B, et al.: Fiji: An open-source platform for biological-image analysis. Nat Methods 2012, 9:676-682.

[14] Thévenaz P, Ruttimann UE, Unser M: A pyramid approach to subpixel registration based on intensity. IEEE Trans Image Process 1998, 7:27-41.

[15] Van Der Walt S, Schönberger JL, Nunez-Iglesias J, Boulogne F, Warner JD, Yager N, Gouillart E, Yu T: Scikit-image: Image processing in python. PeerJ 2014, 2014:1-18.

[16] Moëll MK, Donaldson LA: Shading Correction Methods for Digital Image Analysis of Confocal Wood Images. IAWA J 2007, 28:349-364.

[17] Sweeney TE, Beuchat CA: Limitations of methods of osmometry: measuring the osmolality of biological fluids. Am J Physiol Integr Comp Physiol 1993, 264:R469-R480. 